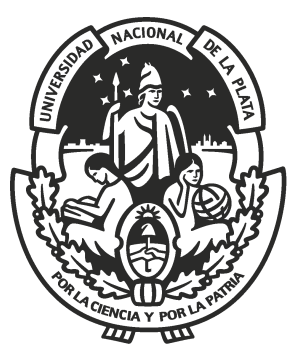

Universidad Nacional de La Plata

Facultad de Ciencias Exactas

Departamento de Matemática

Trabajo de Tesis Doctoral:

Recuperación de fase, perturbación de marcos y un problema tipo Procusto

\title{
Pablo Calderón
}

Director: Mariano Ruiz 



\section{Agradecimientos}

Si bien uno tiene un deseo y hace lo posible por que se cumpla, en mi caso, creo que no hubiera sido posible sin la participación de muchas personas.

En primer lugar, agradezco a mi mamá y a mi viejo por acompañarme en todo momento y por haberme motivado a estudiar desde que tengo uso de razón. Aunque mi viejo se fue antes de que pudiera terminar de escribir, sé que él estaría muy contento.

Agradezco muchísimo a Mariano por haber accedido a dirigirme todos estos años, haberme enseñado a investigar en matemática y siempre estar disponible para hacer alguna cuentita, mates de por medio.

Agradezco al jurado, Celeste Gonzalez, Eduardo Chiumiento y Francisco Martínez Pería, quienes realizaron una lectura minuciosa de la tesis y me han hecho comentarios muy valiosos.

Estoy muy agradecido con el Departamento de Matemática, que cuenta con excelentes docentes de gran calidad humana y cuyo Programa de Retención fue de gran apoyo durante la etapa (pandémica) de escritura de esta tesis. Aprovecho también para agradecer a Claudio de la Secretaría que siempre ha sido de gran ayuda. En este párrafo de agradecimientos institucionales también incluyo al CONICET cuya beca me ha permitido varios de años de formación junto a profesionales de primer nivel en el Instituto Argentino de Matemática.

Agradezco también al grupo de bibliotekids - Noe, Guada, Edus, Seba, Clau y Majo - por la buena compañía en los años de la beca, en especial a Noe Rios, con la que tuve el placer de trabajar y aprender mucho en el último tramo. Hago extensivo este agradecimiento para todos los miembros del IAM con quienes he compartido más de un almuerzo auspiciado por Pizza Pata.

Por último, y no menos importante, agradezco a Dana que me ha acompañado con su amor todo este tiempo, sin ella todo hubiera sido más difícil. 



\section{Introducción}

En esta tesis presentamos los resultados obtenidos en los problemas que derivaron del estudio del problema de recuperación de fase. Principalmente, este trabajo gira entorno a la teoría de marcos, la teoría de operadores y el análisis matricial.

En esencia, el problema de recuperación de fase consiste en la reconstrucción de un objeto general (una señal, una imagen) a partir del módulo de su transformada de Fourier. Esto es, considerando que se tiene la siguiente relación

$$
\left.\widehat{f(u)}=\int_{-\infty}^{+\infty} f(x) \exp (-i 2 \pi u \cdot x)\right) d x=|\widehat{f}(u)| \exp [i \psi(u)]
$$

donde $x$ y $u$ son coordenadas de los espacios de coordenadas y de frecuencias, respectivamente. El objetivo es recuperar $\psi(u)$, o equivalentemente $f(x)$, teniendo al menos $|\widehat{f}(u)|$ como hipótesis inicial [56].

En la década del '60, A. Walther logró resolver el problema para un espacio unidimensional, sugiriendo que sus resultados resultarían de interés para el procesamiento de imágenes [101]. Los posteriores avances sobre el problema fueron a través del desarrollo de algoritmos, sobretodo en el campo de la óptica, para luego extenderse hacia otras áreas. A finales de los '90, los avances tecnológicos y los nuevos formalismos desembocaron en el estudio teórico del problema de recuperación de fase. Expresándolo en forma abstracta, dada una familia de vectores $\mathcal{F}=\left\{f_{n}\right\}_{n=1}^{N}$ de un espacio de Hilbert $\mathcal{H}_{M}$ de dimensión finita $M$, se dice que $\mathcal{F}$ permite recuperación de fase (phase retrieval) si el operador

$$
\mathcal{A}^{\mathcal{F}}: \mathcal{H}_{M} / \sim \rightarrow \mathcal{H}_{N} \text { dado por } \mathcal{A}^{\mathcal{F}}(y):=\left(\left|\left\langle y, f_{n}\right\rangle\right|^{2}\right)_{n}
$$

es inyectivo ( $\sim$ denota la relación de equivalencia dada por $y \sim x$ si y sólo si $y=c x$ para algún escalar $c$ con $|c|=1)$.

A mediados de los '80, luego del trabajo de Daubechies, Grossmann y Meyer [43], se produjo un repentino interés en el estudio de la teoría de marcos que no presentaba visibles avances desde su primer definición en 1952 (ver Breve reseña histórica de marcos). La siguiente es la definición dada por Young en 1980 [104]:

Una sucesión de vectores $\left\{f_{n}\right\}_{n \in I}$ es un marco para un espacio de Hilbert 
$\mathcal{H}$ si existen constantes $A, B>0$ tales que para todo $x \in \mathcal{H}$

$$
A\|x\|^{2} \leq \sum_{n \in I}\left|\left\langle x, f_{n}\right\rangle\right|^{2} \leq B\|x\|^{2}
$$

Este renovado interés se vio favorecido además por el rápido desarrollo de la teoría de wavelets, introduciendo a los marcos como una herramienta muy útil en el análisis tiempo-frecuencia de señales y el procesamiento de imágenes.

En 2005, R. Balan, P. Casazza y D. Edidin publicaron uno de los primeros trabajos de recuperación de fase con un enfoque desde la teoría de marcos [11]. Entre otros resultados, los autores muestran allí que para marcos genéricos $\mathcal{F}$, en el caso real, si $N \geq 2 M-1$ entonces $\mathcal{A}^{\mathcal{F}}$ es inyectivo. En el caso complejo, prueban que $\mathcal{A}^{\mathcal{F}}$ es inyectivo para marcos genéricos con $N \geq 4 M-2$. Cabe mencionar también que conjeturaron que el número óptimo de elementos de $\mathcal{F}$ en el caso complejo debía ser $4 N-4[53,13]$.

Una de las condiciones que se menciona en el trabajo [21] para que un marco provea recuperación de fase es la de full spark.

Un marco $\mathcal{F}$ en un espacio de Hilbert $\mathcal{H}_{M}$ es full spark, si todo subconjunto del marco de cardinal $M$ es linealmente independiente.

Esta característica se menciona en el reciente trabajo [15], donde Bemrose, Casazza, Gröchenig, Lammers y Lynch presentan la noción de marcos woven. Debido a su incipiente estudio, no existen publicaciones que vinculen las nociones de recuperación de fase y woven. Un par de marcos $\mathcal{F}=\left\{f_{i}\right\}_{i \in I}, \mathcal{G}=\left\{g_{i}\right\}_{i \in I}$ para un espacio de Hilbert $\mathcal{H}$ se dice que son woven si existen cotas uniformes de marco para $\left\{f_{i}\right\}_{i \in \sigma} \cup\left\{g_{i}\right\}_{i \in \sigma^{c}}$, para todo $\sigma \subset I$. Realizamos un estudio de pares woven en el Capítulo 2.

En el trabajo [21], J. Cahill, P. Casazza, J. Peterson y L. Woodland presentan una generalización del problema de recuperación de fase en el contexto de los denominados marcos de fusión. Concretamente, el problema es reconstruir un vector a partir de las normas de las proyecciones ortogonales del vector a determinados subespacios. Esto es, dados subespacios cerrados $\left\{W_{k}\right\}_{k=1}^{N}$ de $\mathcal{H}_{M}$, con las medidas $\left\{P_{W_{k}} x\right\}_{k=1}^{N}$ se construye un operador análogo al definido en (1) y se buscan condiciones para que dicho operador sea inyectivo. En el citado trabajo, los autores prueban el notable resultado que la recuperación de fase es posible en $\mathbb{R}^{M}$ usando $2 M-1$ subespacios de dimensión menor a $M-1$.

Un primer paso en el abordaje de los problemas de marcos de fusión con recuperación de fase es estudiar cómo se comportan los marcos bajo perturbaciones. Christensen trabajó en este problema para marcos de vectores en espacios de Hilbert [37] [38]: dado un marco $\mathcal{F}=\left\{f_{i}\right\}_{i \in I}$ para un espacio de Hilbert $\mathcal{H}$ y un operador $T \in L(\mathcal{H})$, se buscan condiciones sobre $T$ de forma que $\left\{T\left(f_{i}\right)\right\}_{i \in I}$ sea también un marco para $\mathcal{H}$. Este problema llevado al contexto de marcos de fusión resultó de interés para varios autores [60] [6] [85]. Destinamos el Capítulo 3 para el estudio de este tipo de perturbaciones. 
Una forma de tratar el problema de recuperación de fase está orientando al uso de algoritmos mediante aproximación matricial [12]. Para esto, consideremos el operador $\mathcal{B}$ definido sobre las matrices hermitianas de dimensión $N \times N$ :

$$
\mathcal{B}: \mathcal{H}^{N \times N} \rightarrow \mathbb{R}^{M}, \mathcal{B}(Y)=\left(f_{n}^{*} Y f_{n}\right)_{n=1}^{M}
$$

Si llamamos $b_{n}=\left|\left\langle y, f_{n}\right\rangle\right|^{2}$ con $n=1, \ldots, N$ a las coordenadas del operador $\mathcal{A}^{\mathcal{F}}$ de (1), tenemos que

$$
b:=\left(\left|\left\langle y, f_{n}\right\rangle\right|^{2}\right)_{n}=\mathcal{B}\left(y y^{*}\right)
$$

Los autores de [26] muestran que el problema de recuperación fase es equivalente a resolver el problema de minimización

$$
\min \{\operatorname{rank}(Y): \mathcal{B}(Y)=b, Y \geq 0\} \text {. }
$$

Como este problema es en general NP-hard, proponen este problema tracial que resulta equivalente a (2) bajo ciertas condiciones:

$$
\operatorname{mín}\{\operatorname{tr}(Y): \mathcal{B}(Y)=b, Y \geq 0\} \text {. }
$$

Problemas similares de minimización de funciones definidas a partir de la traza surgen en el estudio de diseño de marcos, concretamente en los problemas de completaciones óptimas de marcos. En [54], se consideró el siguiente problema: sea $\mathcal{F}_{0}=\left\{f_{i}\right\}_{i=1}^{N_{0}}$ una sucesión de un espacio de Hilbert $M$-dimensional $\mathcal{H}_{M}$ con operador de marco $S_{\mathcal{F}_{0}}$. Sea $a=\left(\alpha_{i}\right)_{i=1}^{k}$ una sucesión de números positivos tales que

$$
r k\left(S_{\mathcal{F}_{0}}\right) \geq M-k \quad \text { y } \quad N=N_{0}+k .
$$

El problema es construir una sucesión $\mathcal{G}=\left\{f_{i}\right\}_{i=N_{0}+1}^{N}$ de $\mathcal{H}_{M}$ tal que

- $\left\|f_{N_{0}+i}\right\|^{2}=\alpha_{i}, 1 \leq i \leq k$.

- $\mathcal{F}=\left\{\mathcal{F}_{0}, \mathcal{G}\right\}=\left\{f_{i}\right\}_{i=1}^{N}$ es un marco que minimiza $\operatorname{tr}\left(S_{\mathcal{F}}^{-1}\right)$.

En [16] estudiaron una variante de este problema y consideraron el potencial de marco $F P(\mathcal{F})=\operatorname{tr}\left(S_{\mathcal{F}}^{2}\right)$ como medida de optimalidad. El estudio de minimización de los potenciales convexos generaliza estos problemas en el trabajo [90]. En esa publicación, los autores consideran funciones convexas y utilizan resultados de mayorización de autovalores para describir los minimizadores de $\operatorname{tr} f\left(S_{\mathcal{F}}\right)$, para $\mathcal{F}=\left\{f_{i}\right\}_{i=1}^{N} \in \mathcal{H}_{M}$. Notar que si $\lambda_{j}\left(S_{\mathcal{F}}\right)$ denota los autovalores del operador de marco $S_{\mathcal{F}}$,

$$
\operatorname{tr} f\left(S_{\mathcal{F}}\right)=\sum_{j=1}^{M} f\left(\lambda_{j}\left(S_{\mathcal{F}}\right)\right) .
$$

Esta serie de relaciones sugieren que el problema de recuperación de fase para marcos se podría estudiar también como un problema de minimización, contando con la 
mayorización de autovalores como herramienta principal y, en particular, las llamadas desigualdades de Lidskii [8] [83].

Un grado mayor de generalidad se puede alcanzar en estos problemas de minimización si se analiza el aspecto local de los minimizadores [89]. En este sentido, las desigualdades de Lidskii nos llevaron a estudiar problemas de cercanía de matrices o problemas de Procusto [64]. En el capítulo 4, nos basamos en el trabajo de Bhatia y Congedo [9] y caracterizamos el siguiente problema de Procusto:

Sea $N(\cdot)$ una norma unitariamente invariante estrictamente convexa $y$ $\mathcal{P}(d)$ el conjunto de las matrices estrictamente positivas de dimensión $d$. Para $A, B \in \mathcal{P}(d)$, si $\mathcal{O}_{B}$ es la órbita unitaria de $B$, se define la distancia $F_{(N, A, B)}=F_{N}: \mathcal{O}_{B} \rightarrow \mathbb{R}$ dada por

$$
F_{N}(C)=N\left(\log \left(A^{-1 / 2} C A^{-1 / 2}\right)\right) \quad \text { para } \quad C \in \mathcal{O}_{B} .
$$

El objetivo es caracterizar las matrices en las que se alcanzan los mínimos

$$
\min _{C \in \mathcal{O}_{B}} F_{N}(C)=\min _{C \in \mathcal{O}_{B}} N\left(\log \left(A^{-1 / 2} C A^{-1 / 2}\right)\right) \text {. }
$$

Utilizando un approach similar al estudio de las desigualdades (aditivas) de Lidskii de [92] caracterizamos los extremos locales de este problema.

\section{Breve reseña histórica de teoría de marcos}

Uno de los primeros antecedentes de la teoría de marcos remite a la década del '30, cuando Paley y Wiener estudiaron las series de Fourier no armónicas

$$
\sum_{n \in \mathbb{Z}} c_{n} e^{i \lambda_{n} t},-\pi \leq t \leq \pi
$$

El problema consistía en buscar condiciones sobre $\left\{\lambda_{n}\right\}_{n \in \mathbb{Z}}$ para que toda función real $f(t)$ corresponda con una expresión sumable del tipo (3) para todo $t \in[-\pi, \pi]$.

En 1946, Dennis Gabor en su Theory of Communication introdujo un método para representar señales unidimensionales en dos dimensiones: tiempo y frecuencia. Más precisamente, él propuso representar $f$ como la serie

$$
\sum_{n, m \in \mathbb{Z}} c_{n, m} g_{m, n}(t)
$$

siendo $g_{m, n}(t)=g(t-n a) e^{2 \pi i m b t}$ y $m, n \in \mathbb{Z}$, para una función fija $g(\cdot)$ y parámetros $a, b>0$ denominados tiempo-frecuencia.

Unos años más tarde, en 1952, Duffin y Schaeffer mostraron que si la sucesión $\left\{\lambda_{n}\right\}_{n \in \mathbb{Z}}$ verificaba un requisito de densidad, la sucesión $\left\{e^{i \lambda_{n}} t\right\}_{n \in \mathbb{Z}}$ no sólo genera el espacio $L^{2}(-\gamma, \gamma)$, con $0 \leq \gamma \leq \pi$, sino que además satisface la siguiente desigualdad 
para toda función $f \in L^{2}(-\gamma, \gamma)$ :

$$
A \int_{-\gamma}^{-\gamma}|f(t)|^{2} d t \leq \frac{1}{2 \pi} \sum_{n}\left|\int_{-\gamma}^{-\gamma} f(t) e^{i \lambda_{n} t} d t\right|^{2} \leq B \int_{-\gamma}^{-\gamma}|f(t)|^{2} d t .
$$

Estos autores denominaron marcos a estas sucesiones [50].

En 1980, R. Young mostró en su libro [104] una fuerte conexión entre las bases de Riesz en un espacio de Hilbert separable $\mathcal{H}$ y el problema de reconstruir $f \in \mathcal{H}$ a partir de

$$
\left\langle f, f_{n}\right\rangle=c_{n}, n=1,2,3, \ldots,
$$

donde $f_{i} \in \mathcal{H}$ y $c_{i}$ son escalares (también conocido como problema de momentos). Young introduce la noción abstracta de marcos relacionándola con este problema y, entre otros resultados, considera aquellos marcos que dejan de serlo si alguno de sus elementos es removido (marcos exactos) y prueba que esta clase de marcos es idéntica a la clase de bases de Riesz. Este problema remite al Problema 8 presentado por Halmos en [66].

Hasta 1985, los marcos limitaban su existencia al contexto de series de Fourier no armónicas. Fue en 1986 que se abrió una puerta para la teoría de marcos con el trabajo fundamental de Daubechies, Grossmann y Meyer [44]. Este fue uno de los precursores trabajos que combinó el análisis de Gabor con la teoría de marcos donde se presentaron resultados de marcos en otros contextos, con implicancias directas en análisis de señales y mecánica cuántica. En términos físicos, probaron que los estados coherentes asociados a reticulados de von Neumann no formaban un marco en $L^{2}(\mathbb{R})$. De forma novedosa, los autores utilizaron los operadores

$T_{a}: L^{2}(\mathbb{R}) \rightarrow L^{2}(\mathbb{R}), T_{a}(f(x))=f(x-a), E_{b}: L^{2}(\mathbb{R}) \rightarrow L^{2}(\mathbb{R}), E_{b}\left(f((x))=e^{2 \pi i b x} f(x)\right.$,

para construir marcos ajustados para $L^{2}(\mathbb{R})$ de la forma $\left\{E_{m b} T_{n a} g\right\}_{m, n \in \mathbb{Z}}$, siendo $g \in$ $L^{2}(\mathbb{R})$ de soporte compacto, en vez de considerar los llamados estados canónicos donde $g$ se tomaba como Gaussiana (como propuso Gabor). Varios autores llaman a este trabajo 'el amanecer de la era wavelet' [17].

Desde entonces, numerosos trabajos sobre marcos se han dado a conocer en distintos contextos, entre ellos podemos mencionar el trabajo de K. Gröchenig sobre marcos en espacios Banach [65], el de M. Frank y D. Larson sobre marcos en módulos de Hilbert [59] y el de J. Giribet, A. Maestripieri, F. Martínez Pería y P. Massey en espacios de Krein [62]. Más allá de los vectores, los marcos han sido generalizados a subespacios, inicialmente en el trabajo de P. Casazza y G. Kutyniok [29], y luego renombrados por los mismos autores y S. Li como marcos de fusión en [31] (ver Capítulo 4). Esta extensión de la definición de marcos a subspacios, así como los pseudo-marcos [81], los marcos oblicuos [40] [51] y los marcos externos [1], fueron condensados en los g-marcos de G. Sun en [98]. Debido a la estrecha relación con el campo aplicado la teoría de marcos se encuentra en constante crecimiento hasta la actualidad. 


\section{Descripción de los contenidos de la tesis}

Realizamos una concisa descripción de los resultados de esta tesis que principalmente provienen de los trabajos [22] [23] [24] [25].

Capítulo 1. Enunciamos las definiciones, resultados y notaciones que se utilizarán a lo largo de esta tesis. El ángulo entre subespacios, el módulo mínimo y el gap entre subespacios son las las herramientas que más utilizaremos. Definimos la noción de marcos para vectores, y su generalización a subespacios. Presentamos también los resultados de mayorización que serán necesarios para el último capítulo, junto con una breve sección de geometría diferencial.

Capítulo 2. En este capítulo estudiamos relaciones entre el gap entre subespacios cerrados y conjuntos de vectores que sean sucesiones de Riesz o sucesiones marcos. En el Lema 2.1.7, probamos que si una sucesión es sucesión de Riesz es equivalente a que su operador grammiano esté acotado inferiormente por un múltiplo de la identidad. Asimismo, una sucesión es sucesión marco si y sólo si su operador de marco acota superiormente un múltiplo de una proyección. La sección 2.2 comienza con una forma de calcular el gap que provee de varias equivalencias entre las que se destaca el Teorema 2.2.4. En la última sección aplicamos nuestros resultados a marcos de Parseval: por un lado, consideramos un resultado de Olevskii y Ulanovskii donde proyectan una base ortonormal en bloques y prueban que sus imágenes son sucesiones marco o sucesiones de Riesz, extendemos dicho resultado con una prueba alternativa en el Teorema 2.3.2, donde mejoramos la cota de las sucesiones; por otro lado, en la Proposición 2.3.5 adaptamos un resultado de Bodmann, Casazza, Paulsen y Speegle para caracterizar las subfamilias de un marco de Parseval que sean marco.

Capítulo 3. Estudiamos un caso particular de los llamados marcos woven. El Teorema 3.2.4 de Bemrose, Casazza, Gröchenig, Lammers y Lynch muestra que, a partir de una desigualdad de las cotas de marco de un par $(\mathcal{F}, \mathcal{G})$, se puede concluir que ese par es woven. Generalizamos estos resultados en el Teorema 3.2.7 y su Corolario 3.2.10. Como aplicación del Teorema 3.2.7 obtenemos una condición para obtener pares woven con el marco dual canónico de un marco dado. Posteriormente, probamos una caracterización de pares woven utilizando el ángulo entre subespacios en el Teorema 3.4.4, un resultado de perturbación en el Teorema 3.2.12 y una relación de transitividad entre pares woven en la Proposición 3.5.2.

Capítulo 4. Este capítulo se centra en perturbación de marcos de fusión, esto es, dado un marco de fusión $W=\left\{W_{i}\right\}_{i \in I}$ y un operador $T \in L(\mathcal{H})$, nos interesa que $\left\{T\left(W_{i}\right)\right\}_{i \in I}$ sea un marco de fusión. Damos un primer resultado de perturbación de marcos de vectores con ángulos en la Proposición 4.1.7. El Teorema 4.2.6 de X. Li et al propone una condición de gap de subespacios sobre el operador de perturbación. En el camino de mejorar ese resultado probamos una equivalencia de perturbación por operadores de rango cerrado que involucra el módulo mínimo, el gap y el ángulo entre subespacios (Teorema 4.2.8 y su Corolario 4.2.9). En el Teorema 4.2.10, generalizamos el resultado de Li y exhibimos cotas para los pesos de los subespacios. 
Capítulo 5. En [9], Bhatia y Congedo proponen un problema de Procusto donde prueban que los mínimos se alcanzan en aquellas matrices que satisfacen una condición de conmutatividad. Siguiendo las técnicas de [93], realizamos los cálculamos en la versión multiplicativa local de las desigualdades de Lidskii y en el Teorema 5.3.2 completamos el resultado principal de [9]. Esto es, mostramos que los minimizadores locales para el problema de Procusto sólo pueden ser aquellos puntos de la órbita propuestos en ese trabajo. Más aún, los mínimos locales resultan globales y no dependen de la norma unitariamente invariante empleada en el problema. 



\section{Índice}

1. Preliminares 1

1.1. Ángulos entre subespacios y módulo mínimo reducido . . . . . . . . . 1

1.2. Gap entre subespacios . . . . . . . . . . . . . . 3

1.2.1. Gap, ángulos y proyecciones . . . . . . . . . . . 5

1.3. Marcos de vectores . . . . . . . . . . . . . . 6

1.4. Marcos de fusión .................... 8

1.5. Matrices y autovalores . . . . . . . . . . . . . 9 9

1.5.1. Mayorización . . . . . . . . . . . . . 10

1.5.2. Normas unitariamente invariantes . . . . . . . . . . . . . . 11

1.5.3. Desigualdades de Lidskii . . . . . . . . . . . . . . 13

1.5.4. Aspectos locales . . . . . . . . . . . . . . . 14

2. Marcos y sucesiones de Riesz $\quad \mathbf{1 7}$

2.1. Sucesiones de Riesz . . . . . . . . . . . . . . . . . . 18

2.1.1. Sucesiones de Riesz y sucesiones marco . . . . . . . . . . . 19

2.2. Marcos y gap entre subespacios . . . . . . . . . . . . . 20

2.3. Aplicaciones del gap a marcos de Parseval . . . . . . . . . . . . . . 23

2.3.1. Sucesiones de Riesz y sucesiones marco . . . . . . . . . . . 24

2.3.2. Condición del espectro del Grammiano . . . . . . . . . . . 26

3. Marcos Woven 29

3.1. Definición de woven y principales resultados . . . . . . . . . . 30

3.2. Pares woven y operadores . . . . . . . . . . . . . 31

3.2.1. Condiciones suficientes . . . . . . . . . . . . . . . . . . . . . . . . . . . 32

3.2.2. Gap y pares woven . . . . . . . . . . . . 37

3.3. Caso particular: marco dual canónico escaleado . . . . . . . . . . . . . 38

3.4. Condición necesaria de ángulos . . . . . . . . . . . . . 40

3.5. Una condición de transitividad . . . . . . . . . . . . . . . . . . . . 42

4. Perturbación de marcos de fusión $\quad \mathbf{4 5}$

4.1. Perturbación de marcos vectoriales . . . . . . . . . . . . 46

4.1.1. Bases y sucesiones de Bessel . . . . . . . . . . . . . . . . . 46

4.1.2. Marcos de vectores . . . . . . . . . . . . . . 47

4.2. Perturbación de marcos de fusión . . . . . . . . . . . . . . . 49 
4.2.1. Antecedentes de perturbación . . . . . . . . . . . 50

4.2.2. El gap de subespacios cerrados perturbados . . . . . . . 53

4.2.3. Variación de pesos . . . . . . . . . . . . . . 55

4.3. Sobre perturbaciones efectivas de marcos de fusión $\mathrm{RF} \ldots \ldots \ldots$

5. Extremos locales para problemas tipo Procusto 61

5.1. Lidskii como un problema de optimalidad . . . . . . . . . . . 62

5.1.1. Diseño de $\operatorname{marcos} \ldots \ldots \ldots$. . . . . . . . . . . 63

5.1.2. Desigualdad aditiva y minimizadores globales . . . . . . . 64

5.1.3. Versión multiplicativa de las desigualdades . . . . . . . . 65

5.2. Desigualdades de Lidskii locales . . . . . . . . . . . . 66

5.2.1. Desigualdad aditiva local . . . . . . . . . . . . . 67

5.2.2. Desigualdad multiplicativa local . . . . . . . . . . 68

5.3. Caracterización de extremos locales en un problema de Procusto . . 74

5.3.1. Motivación: caso ortogonal . . . . . . . . . . . . . . 74

5.3.2. Distancias $\log \ldots \ldots \ldots \ldots \ldots \ldots \ldots \ldots$

5.3.3. Caracterización de los mínimos locales . . . . . . . . 76 


\section{Abreviaturas y símbolos}

$$
\begin{array}{cl}
\text { NUI } & \text { : norma unitariamente invariante } \\
A_{\mathcal{F}} & \text { : cota óptima inferior del marco } \mathcal{F} \\
B_{\mathcal{F}} & \text { : cota de marco superior del marco } \mathcal{F} \\
A_{\sigma} & \text { : cota óptima inferior de un weaving } \\
B_{\sigma} & \text { : cota óptima superior de un weaving }
\end{array}
$$

$c(\mathcal{M}, \mathcal{N})$ : coseno del ángulo entre los subespacios $\mathcal{M}$ y $\mathcal{N}$

$D_{x} \quad$ : matriz diagonal con diagonal principal $x$

$\mathcal{F}_{I} \quad$ : elementos de índice $I$ de la sucesión $\mathcal{F}$

$\gamma(T)$ : módulo mínimo de un operador $T$

$G_{\mathcal{F}} \quad$ : operador grammiano de un marco $\mathcal{F}$

$\mathbb{I}_{d} \quad:\{1, \ldots, d\}$

$L(\mathcal{H})$ : operadores acotados de un espacio de Hilbert $\mathcal{H}$

$\mathcal{M}_{k, d}(\mathbb{C})$ : espacio de matrices complejas de dimensión $k \times d$

$N(T)$ : núcleo de un operador $T$

$\mathcal{O}_{A} \quad$ : órbita unitaria de $A$

$\mathcal{P}(d)$ : matrices estrictamente positivas de dimensión $d$

$P_{\mathcal{M}} \quad$ : proyección ortogonal sobre el subespacio $\mathcal{M}$

$R(T)$ : rango de un operador $T$

$s(\mathcal{M}, \mathcal{N})$ : seno del ángulo entre los subespacios $\mathcal{M}$ y $\mathcal{N}$

$\mathcal{S}^{\prime} \quad$ : conmutante de $\mathcal{S}$

$S_{\mathcal{F}} \quad$ : operador de marcos de un marco $\mathcal{F}$

$S_{\mathcal{W}_{w}}$ : operador de marco de un marco de fusión $\mathcal{W}_{w}$

$T^{\dagger} \quad$ : pseudoinversa de Moore-Penrose de $T$

$T_{\mathcal{F}} \quad$ : operador de síntesis de un marco $\mathcal{F}$

$T_{\mathcal{F}}^{*} \quad$ : operador de análisis de un marco $\mathcal{F}$

$T_{\mathcal{W}_{w}}$ : operador de análisis de un marco de fusión $\mathcal{W}_{w}$

$\mathcal{W}_{w} \quad$ : familia de subespacios $\mathcal{W}$ con pesos $w$ 



\section{Capítulo 1}

\section{Preliminares}

En este capítulo introducimos las nociones y resultados que utilizaremos a lo largo de esta tesis. Principalmente, $\mathcal{H}$ y $\mathcal{K}$ representarán espacios de Hilbert separables dotados con un producto interno $\langle.,$.$\rangle . Al espacio de las transformaciones lineales$ acotadas de $\mathcal{H}$ en $\mathcal{K}$ lo denotaremos por $L(\mathcal{H}, \mathcal{K})$ y por $L(\mathcal{H})$ en el caso que $\mathcal{H}=\mathcal{K}$. Dado $T \in L(\mathcal{H}, \mathcal{K}), R(T)$ denota la imagen de $T$ en $\mathcal{K}$, y $N(T)$ el núcleo en $\mathcal{H}$.

En $L(\mathcal{H}, \mathcal{K})$ consideramos la norma de operadores definida por

$$
\|B\|=\sup \{\|B x\|: x \in \mathcal{H},\|x\|=1\}=\inf \{\alpha>0:\|B x\| \leq \alpha\|x\| \forall x \in \mathcal{H}\} .
$$

Los operadores positivos serán denotados por

$$
L(\mathcal{H})^{+}=\{B \in L(\mathcal{H}):\langle B x, x\rangle \geq 0, \forall x \in \mathcal{H}\}
$$

y por $U(\mathcal{H})$ a los operadores unitarios de $\mathcal{H}$. Dado un subespacio cerrado $\mathcal{M}$ de $\mathcal{H}$, denotaremos por $P_{\mathcal{M}}$ a su proyección ortogonal.

\section{1. Ángulos entre subespacios y módulo mínimo re- ducido}

Consideramos dos nociones de ángulos entre subespacios cerrados de $\mathcal{H}$, debidas a Friedrichs y Dixmier, que las denominaremos ángulo y ángulo minimo, respectivamente.

Sean dos subespacios cerrados $\mathcal{M}$ y $\mathcal{N}$, el ángulo entre $\mathcal{M}$ y $\mathcal{N}$ es el ángulo de $[0, \pi / 2]$ cuyo coseno se define por

$$
c(\mathcal{M}, \mathcal{N})=\sup \{|\langle x, y\rangle|: x \in \mathcal{M} \ominus \mathcal{N}, y \in \mathcal{N} \ominus \mathcal{M} \text { y }\|x\|=\|y\|=1\},
$$

siendo $\mathcal{M} \ominus \mathcal{N}=\mathcal{M} \cap(\mathcal{M} \cap \mathcal{N})^{\perp}$. Por tanto, el seno de este ángulo se denota $s(\mathcal{M}, \mathcal{N})=\left(1-c(\mathcal{M}, \mathcal{N})^{2}\right)^{1 / 2}$.

Por otro lado, el ángulo mínimo entre $\mathcal{M}$ y $\mathcal{N}$ es el ángulo de $[0, \pi / 2]$ cuyo 
coseno se define por

$$
c_{0}(\mathcal{M}, \mathcal{N})=\sup \{|\langle x, y\rangle|: x \in \mathcal{M}, y \in \mathcal{N} \text { y }\|x\|=\|y\|=1\}
$$

La noción de ángulo mínimo probablemente sea la forma más natural de considerar el ángulo entre subespacios. El problema de esta noción de ángulo es que si los subespacios tienen intersección (no trivial) el ángulo sería nulo, lo que no sucede con el ángulo de Friedrichs. Otra ventaja de este último es que satisface que

$$
c(\mathcal{M}, \mathcal{N})=c(\mathcal{M} \ominus \mathcal{N}, \mathcal{N})
$$

por lo que se puede reducir el cálculo del ángulo al caso donde los subespacios no tienen intersección no trivial. Más detalles se pueden encontrar en el famoso trabajo de Deutsch [47].

Proposición 1.1.1. Sean dos subespacios cerrados $\mathcal{M}$ y $\mathcal{N}$ de $\mathcal{H}$.

1. $c(\mathcal{M}, \mathcal{N})=c(\mathcal{N}, \mathcal{M})=c(\mathcal{M} \ominus \mathcal{M}, \mathcal{N})=c(\mathcal{M}, \mathcal{N} \ominus \mathcal{M})$.

2. Si $\operatorname{dim}(\mathcal{M})<\infty$, entonces $c(\mathcal{M}, \mathcal{N})<1$.

3. $c(\mathcal{M}, \mathcal{N})<1$ si y sólo si $M+N$ es cerrado.

4. $c(\mathcal{M}, \mathcal{N})=c\left(\mathcal{M}^{\perp}, \mathcal{N}^{\perp}\right)$.

5. $c(\mathcal{M}, \mathcal{N})=\left\|P_{\mathcal{M}} P_{\mathcal{N} \ominus \mathcal{M}}\right\|=\left\|P_{\mathcal{M} \ominus \mathcal{N}} P_{\mathcal{N}}\right\|=\left\|P_{\mathcal{M}} P_{\mathcal{N}}-P_{\mathcal{M} \cap \mathcal{N}}\right\|$.

El producto de proyecciones ortogonales permite calcular el ángulo entre los subespacios:

Proposición 1.1.2. Sea $\mathcal{M}$ y $\mathcal{N}$ subespacios cerrados de $\mathcal{H}$. Entonces

$$
c(\mathcal{M}, \mathcal{N})=\left\|P_{\mathcal{M}} P_{\mathcal{N}} P_{(\mathcal{M} \cap \mathcal{N})^{\perp}}\right\| \text { y } c_{0}(\mathcal{M}, \mathcal{N})=\left\|P_{\mathcal{M}} P_{\mathcal{N}}\right\|=\left\|P_{\mathcal{M}} P_{\mathcal{N}} P_{\mathcal{M}}\right\|^{1 / 2}
$$

Se pueden considerar a las proyecciones ortogonales como miembros de una clase más grande de operadores: los operadores de rango cerrado. Es trivial que el producto de operadores rango cerrado no necesariamente tiene rango cerrado. Por medio de una cota del ángulo se puede caracterizar a aquellos productos que sí verifican esta propiedad.

Para un operador $T \in L(\mathcal{H})$, podemos definir su módulo mínimo reducido $\gamma(T)$, dado por

$$
\gamma(T):=\inf \left\{\|T x\| \mid\|x\|=1, x \in N(T)^{\perp}\right\} .
$$

Es un resultado conocido que si $T$ tiene rango cerrado,

$$
\gamma(T)=\gamma\left(T^{*}\right)=\gamma\left(T^{*} T\right)^{1 / 2}=\left\|T^{\dagger}\right\|^{-1}
$$

siendo $T^{\dagger}$ la pseudoinversa de Moore-Penrose. Además, 
Proposición 1.1.3. Un operador $T \in L(\mathcal{H})$ tiene rango cerrado si y sólo si $\gamma(T)>0$.

Las siguientes desigualdades relacionan las nociones de ángulo y módulo mínimo reducido de operadores en el contexto de operadores de rango cerrado. Estos resultados provienen de [3] y [4].

Proposición 1.1.4. Sean $A, B \in L(\mathcal{H})$ de rango cerrado. Entonces

$$
\gamma(A) \gamma(B) s(N(A), R(B)) \leq \gamma(A B) \leq\|A\|\|B\| s(N(A), R(B)) .
$$

En particular, si $T \in L(\mathcal{H})$ es un operador de rango cerrado y $\mathcal{M}$ es un subespacio cerrado de $\mathcal{H}$ con $c(N(T), \mathcal{M})<1$, entonces

$$
\gamma(T) s(N(T), \mathcal{M}) \leq \gamma\left(T P_{\mathcal{M}}\right) \leq\|T\| s(N(T), \mathcal{M})
$$

El anterior resultado permite caracterizar, a partir de operadores de rango cerrado, los productos de operadores que tengan rango cerrado:

Proposición 1.1.5. Sean $A, B \in L(\mathcal{H})$ operadores de rango cerrado. Entonces, $A B$ tiene rango cerrado si y sólo si $c(R(B), N(A))<1$.

\subsection{Gap entre subespacios}

Entre las diversas formas de "medir subespacios"(e.g. distancias, ángulos) podemos encontrar el gap o apertura entre subespacios. Esta noción, introducida por T. Kato en 1958 [75], si bien no es una distancia, en algunas aplicaciones resulta más fácil de calcular que las distancias usuales.

Uno de los problemas famosos vinculados a esta cantidad es el estudio de pares de subespacios cerrados $\mathcal{M}, \mathcal{N}$ de un espacio de Banach, para los cuales la suma $\mathcal{M}+\mathcal{N}$ es cerrada. Kato plantea al gap como una forma de medir la distancia entre los gráficos de dos operadores de rango cerrado de un espacio de Banach. De esta manera, se pueden estudiar los operadores denominados estables bajo perturbación acotada, esto es, aquellos operadores de rango cerrado $A$ tales que para un operador acotado $T$, el operador $A+T$ sea un operador de rango cerrado.

Dado que en esta tesis nos centramos en marcos en espacios de Hilbert, nos centraremos en resultados relativos al gap definido en este contexto.

Definición 1.2.1. Dados dos subespacios cerrados $\mathcal{M}$ y $\mathcal{N}$ de un espacio de Hilbert $\mathcal{H}$, el gap se define como

$$
\delta(\mathcal{M}, \mathcal{N})=\sup _{x \in \mathcal{M},\|x\| \leq 1} \operatorname{dist}(x, \mathcal{N})
$$

La siguiente proposición es una alternativa a la definición para calcular el gap. Se aprecia en este resultado una relación de igualdad del gap entre subespacios con el gap de los subespacios ortogonales. 
Proposición 1.2.2. Sean $\mathcal{M}, \mathcal{N}$ subespacios cerrados de $\mathcal{H}$, entonces

$$
\delta(\mathcal{M}, \mathcal{N})=\left\|P_{\mathcal{N}^{\perp}} P_{\mathcal{M}}\right\|=\left\|P_{\mathcal{M}} P_{\mathcal{N}^{\perp}} P_{\mathcal{M}}\right\|^{1 / 2}
$$

En particular,

$$
\delta(\mathcal{M}, \mathcal{N})=\delta\left(\mathcal{N}^{\perp}, \mathcal{M}^{\perp}\right)
$$

Demostración. Dado que $\operatorname{dist}(x, \mathcal{N})=\left\|P_{\mathcal{N}^{\perp}} x\right\|$ para todo $x \in \mathcal{H}$, tenemos que

$$
\delta(\mathcal{M}, \mathcal{N})=\sup _{x \in \mathcal{M},\|x\| \leq 1}\left\|P_{\mathcal{N} \perp} x\right\|=\sup _{x \in \mathcal{M},\|x\| \leq 1}\left\|P_{\mathcal{N}^{\perp}} P_{\mathcal{M}} x\right\|
$$

Luego,

$$
\delta(\mathcal{M}, \mathcal{N}) \leq \sup _{\|x\| \leq 1}\left\|P_{\mathcal{N}^{\perp}} P_{\mathcal{M}} x\right\| \leq\left\|P_{\mathcal{N}^{\perp}} P_{\mathcal{M}}\right\|
$$

Por otro lado, dado $x \in \mathcal{H}$ con $\|x\| \leq 1$,

$$
\left\|P_{\mathcal{N}^{\perp}} P_{\mathcal{M}} x\right\|=\operatorname{dist}\left(P_{\mathcal{M}} x, \mathcal{N}\right)
$$

y tomando supremos,

$$
\left\|P_{\mathcal{N}^{\perp}} P_{\mathcal{M}}\right\| \leq \delta(\mathcal{M}, \mathcal{N})
$$

Es fácil ver que en general $\delta(\mathcal{M}, \mathcal{N}) \neq \delta(\mathcal{N}, \mathcal{M})$. Notar que si $\mathcal{M} \subset \mathcal{N}$ y existe $q \in \mathcal{N}$ tal que $\operatorname{dist}(q, \mathcal{M})>0$, se tiene que $\delta(\mathcal{M}, \mathcal{N})=0$ pero $\delta(\mathcal{N}, \mathcal{M})>0$.

Para obtener una métrica con esta noción, se puede considerar el máximo entre $\delta(\mathcal{M}, \mathcal{N})$ y $\delta(\mathcal{N}, \mathcal{M})$

$$
\widehat{\delta}(\mathcal{M}, \mathcal{N})=\operatorname{máx}\{\delta(\mathcal{M}, \mathcal{N}), \delta(\mathcal{N}, \mathcal{M})\}
$$

Con esta definición ahora es correcto afirmar que

$$
\widehat{\delta}(\mathcal{M}, \mathcal{N})=\widehat{\delta}(\mathcal{N}, \mathcal{M})
$$

El hecho que $\widehat{\delta}(\mathcal{M}, \mathcal{N})$ sea una distancia en la grassmanniana se deduce inmediatamente del siguiente resultado.

Teorema 1.2.3. (Kato) Sean $\mathcal{M}, \mathcal{N}$ subespacios cerrados de $\mathcal{H}$, entonces

$$
\widehat{\delta}(\mathcal{M}, \mathcal{N})=\left\|P_{\mathcal{M}}-P_{\mathcal{N}}\right\|
$$

Demostración. Por el resultado anterior, $\operatorname{como} \delta(\mathcal{M}, \mathcal{N})=\left\|P_{\mathcal{N}^{\perp}} P_{\mathcal{M}}\right\|$,

$$
\widehat{\delta}(\mathcal{M}, \mathcal{N})^{2}=\operatorname{máx}\left\{\left\|P_{\mathcal{N}^{\perp}} P_{\mathcal{M}} P_{\mathcal{N}^{\perp}}\right\|, \| P_{\mathcal{N}} P_{\mathcal{M}^{\perp}} P_{\mathcal{N}}\right\} \| \text {. }
$$


Ahora, por la ortogonalidad entre $\mathcal{N}$ y $\mathcal{N}^{\perp}$

$$
\left\|P_{\mathcal{N}^{\perp}} P_{\mathcal{M}} P_{\mathcal{N}^{\perp}}+P_{\mathcal{N}} P_{\mathcal{M}^{\perp}} P_{\mathcal{N}}\right\|=\operatorname{máx}\left\{\left\|P_{\mathcal{N}^{\perp}} P_{M} P_{\mathcal{N}^{\perp}}\right\|,\left\|P_{\mathcal{N}} P_{\mathcal{M}^{\perp}} P_{N}\right\|\right\}
$$

Luego,

$$
\begin{aligned}
\left\|P_{\mathcal{N}^{\perp}} P_{\mathcal{M}} P_{\mathcal{N}^{\perp}}+P_{\mathcal{N}} P_{\mathcal{M}^{\perp}} P_{\mathcal{N}}\right\|= & \left\|\left(I-P_{\mathcal{N}}\right) P_{\mathcal{M}}\left(I-P_{\mathcal{N}}\right)+P_{\mathcal{N}}\left(I-P_{\mathcal{M}}\right) P_{\mathcal{N}}\right\| \\
= & \| P_{\mathcal{M}}-P_{\mathcal{N}} P_{\mathcal{M}}-P_{\mathcal{M}} P_{\mathcal{N}}+ \\
& \quad+P_{\mathcal{N}} P_{\mathcal{M}} P_{\mathcal{N}}+P_{\mathcal{N}}-P_{\mathcal{N}} P_{\mathcal{M}} P_{\mathcal{N}} \| \\
= & \left\|P_{\mathcal{M}}+P_{\mathcal{N}}-P_{\mathcal{N}} P_{\mathcal{M}}-P_{\mathcal{M}} P_{\mathcal{N}}\right\| \\
= & \left\|\left(P_{\mathcal{M}}-P_{\mathcal{N}}\right)\left(P_{\mathcal{M}}-P_{\mathcal{N}}\right)\right\| \\
= & \left\|P_{\mathcal{M}}-P_{\mathcal{N}}\right\| .
\end{aligned}
$$

\subsubsection{Gap, ángulos y proyecciones}

Dado que se pueden calcular el ángulo y el gap entre subespacios tomando la norma de las proyecciones, es natural observar relaciones entre estas cantidades.

Sean $\mathcal{M}, \mathcal{N}$ subespacios cerrados de $\mathcal{H}$, de la Proposición 1.2.2, se tiene que

$$
\delta(\mathcal{M}, \mathcal{N})=c_{0}\left(\mathcal{M}, \mathcal{N}^{\perp}\right)
$$

Por otro lado, si $\mathcal{M} \cap \mathcal{N}^{\perp}=\{0\}$,

$$
\delta(\mathcal{M}, \mathcal{N})=c\left(\mathcal{M}, \mathcal{N}^{\perp}\right)
$$

Estas relaciones permiten caracterizar a los operadores $P_{\mathcal{N}} P_{\mathcal{M}}$ que tienen rango cerrado con el gap.

Proposición 1.2.4. Sean $\mathcal{M}, \mathcal{N}$ subespacios cerrados de $\mathcal{H}$,

$$
\delta(\mathcal{M}, \mathcal{N})<1 \text { implica que } P_{\mathcal{N}} P_{\mathcal{M}} \text { tiene rango cerrado }
$$

Demostración. Si $\delta(\mathcal{M}, \mathcal{N})<1$ se tiene que $\mathcal{M} \cap \mathcal{N}^{\perp}=\{0\}$ y por lo tanto $c\left(\mathcal{M}, \mathcal{N}^{\perp}\right)=$ $\delta(\mathcal{M}, \mathcal{N})$. Por la Proposición 1.1.5, $c\left(\mathcal{M}, \mathcal{N}^{\perp}\right)=c\left(R\left(P_{\mathcal{M}}\right), N\left(P_{\mathcal{N}}\right)\right)<1$ implica que $P_{\mathcal{N}} P_{\mathcal{M}}$ tiene rango cerrado.

El siguiente es un conocido resultado de operadores que nos permitirá probar durante la tesis algunas equivalencias entre el gap, el ángulo entre subespacios y sus proyecciones ortogonales.

Teorema 1.2.5. (Douglas) [49] Sean $B \in L(\mathcal{H}, \mathcal{K})$ y $C \in L(\mathcal{G}, \mathcal{K})$. Las siguientes condiciones son equivalentes:

1. Existe $D \in L(\mathcal{G}, \mathcal{H})$ tal que $B D=C$. 
2. $R(C) \subset R(B)$.

3. Existe $a>0$ tal que $C C^{*} \leq a B B^{*}$.

\subsection{Marcos de vectores}

En esta sección presentamos todas las definiciones y resultados generales relacionados con la teoría de marcos. Enunciamos las definiciones para un conjunto de índices numerable, de forma de abarcar los distintos tipos de marcos que se consideran en esta tesis. Se considerarán especialmente sucesiones finitas $\left\{f_{k}\right\}_{k=1}^{N}$ para espacios de Hilbert de dimensión finita $\mathcal{H}_{M}$, así como sucesiones sobre $\mathbb{N}$. Las definiciones, tanto de marcos como de sus operadores, se enuncian de forma análoga.

Definición 1.3.1. Sea $\mathcal{F}=\left\{f_{k}\right\}_{k \in I}$ una sucesión de vectores en un espacio de Hilbert separable $\mathcal{H}$.

1. $\mathcal{F}$ es una sucesión de Bessel para $\mathcal{H}$ si existe $B>0$ tal que para todo $x \in \mathcal{H}$,

$$
\sum_{k \in I}\left|\left\langle x, f_{k}\right\rangle\right|^{2} \leq B\|x\|^{2}
$$

2. $\mathcal{F}$ es un marco para $\mathcal{H}$ si existen dos constantes positivas $A$ y $B$ tales que para todo $x \in \mathcal{H}$,

$$
A\|x\|^{2} \leq \sum_{k \in I}\left|\left\langle x, f_{k}\right\rangle\right|^{2} \leq B\|x\|^{2}
$$

3. Las cotas $A$ y $B$ que verifican (1.6) se denominan cotas de marco. Las cotas de marco óptimas las denotamos por $A_{\mathcal{F}}$ y $B_{\mathcal{F}}$, es decir, $A_{\mathcal{F}}$ es el supremo de las cotas de marco inferiores y $B_{\mathcal{F}}$ es el infimo de las cotas de marco superiores. Si $A_{\mathcal{F}}=B_{\mathcal{F}}$ el marco se dice ajustado, si además $A_{\mathcal{F}}=B_{\mathcal{F}}=1$ se lo llama de Parseval.

4. $\mathcal{F}$ es una sucesión marco para $\mathcal{H}$ si $\mathcal{F}$ es un marco para $\overline{\operatorname{span}}\left\{f_{k}\right\}_{k \in I}$.

Se definen una serie de operadores que permiten caracterizar a aquellas sucesiones que son marcos. En primer lugar, consideramos el operador de síntesis

$$
T_{\mathcal{F}}: \ell^{2}(I) \rightarrow \mathcal{H}, T_{\mathcal{F}}(c)=\sum_{k \in I} c_{k} f_{k}
$$

y el operador de análisis,

$$
T_{\mathcal{F}}^{*}: \mathcal{H} \rightarrow \ell^{2}(I),, T_{\mathcal{F}}^{*}(x)=\left\{\left\langle x, f_{k}\right\rangle\right\}_{k \in I} .
$$

Notar que están bien definidos por (1.5) y son uno el adjunto del otro.

Proposición 1.3.2. [38] Sea $\mathcal{F}=\left\{f_{k}\right\}_{k \in I}$ una sucesión de Bessel en un espacio de Hilbert separable $\mathcal{H}$. 
1. $\mathcal{F}$ es una sucesión marco si y sólo si el rango del operador de síntesis $R\left(T_{\mathcal{F}}\right)$ es cerrado.

2. $\mathcal{F}$ es un marco para $\mathcal{H}$ si y sólo si $R\left(T_{\mathcal{F}}\right)=\mathcal{H}$.

3. Las cotas óptimas del marco son

$$
A_{\mathcal{F}}=\gamma\left(T_{\mathcal{F}}\right)^{2} \quad y \quad B_{\mathcal{F}}=\left\|T_{\mathcal{F}}\right\|^{2}
$$

Una generalización de este resultado se obtuvo en [4]:

Proposición 1.3.3. Sea $\mathcal{F}=\left\{f_{n}\right\}_{n \in \mathbb{N}}$ un marco para un espacio de Hilbert $\mathcal{H}$. Sea $\left\{e_{n}\right\}_{n \in \mathbb{N}}$ la base canónica ortonormal de $\ell^{2}(\mathbb{N})$. Si $I \subset \mathbb{N}$, sea $P_{I}$ la proyección ortogonal sobre $\overline{\operatorname{span}}\left\{e_{n}: n \in I\right\}$ y $\mathcal{F}_{I}:=\left\{f_{n}\right\}_{n \in I}$. Entonces:

1. $\mathcal{F}_{I}$ es una sucesión marco si y sólo si $R\left(T_{\mathcal{F}} P_{I}\right)$ es cerrado.

2. $\mathcal{F}_{I}$ es un marco si y sólo si $R\left(T_{\mathcal{F}} P_{I}\right)=\mathcal{H}$.

En ambos casos, las cotas de marco para $\mathcal{F}_{I}$ son $A=\gamma\left(T_{\mathcal{F}} P_{I}\right)^{2}$ y $B=\left\|T_{\mathcal{F}} P_{I}\right\|^{2}$.

Definidos los anteriores operadores, para un marco $\mathcal{F}$ podemos considerar el operador de marco,

$$
S_{\mathcal{F}}=T_{\mathcal{F}} T_{\mathcal{F}}^{*}: \mathcal{H} \rightarrow \mathcal{H}, S_{\mathcal{F}}(x)=\sum_{k \in I}\left\langle x, f_{k}\right\rangle f_{k}
$$

Este operador, que resulta claramente autoadjunto e inversible por (1.6), verifica que

$$
A_{\mathcal{F}} I \leq S_{\mathcal{F}} \leq B_{\mathcal{F}} I
$$

Además permite reconstruir vectores $x$ de $\mathcal{H}$ :

$$
x=S_{\mathcal{F}} S_{\mathcal{F}}^{-1}(x)=\sum_{k \in I}\left\langle x, S_{\mathcal{F}}^{-1}\left(f_{k}\right)\right\rangle f_{k}
$$

y también

$$
x=S_{\mathcal{F}}^{-1} S_{\mathcal{F}}(x)=\sum_{k \in I}\left\langle x, f_{k}\right\rangle S_{\mathcal{F}}^{-1}\left(f_{k}\right) .
$$

La sucesión $\left\{S_{\mathcal{F}}^{-1} f_{k}\right\}_{k \in I}$ es un marco al que se lo denomina marco dual canónico. Sin embargo, este no es necesariamente el único marco que verifica (1.9) y (1.10), lo que lleva a considerar la siguiente definición:

Definición 1.3.4. Sea $\mathcal{F}$ un marco para $\mathcal{H}$. Un marco $\mathcal{G}=\left\{g_{k}\right\}_{k \in I}$ es un marco dual para $\mathcal{F}$ si satisface las siguientes igualdades para todo $x \in \mathcal{H}$ :

$$
x=\sum_{k \in I}\left\langle x, g_{k}\right\rangle f_{k}=\sum_{k \in I}\left\langle x, f_{k}\right\rangle g_{k} .
$$


De entre todos los marcos duales $\mathcal{G}=\left\{g_{k}\right\}_{k \in I}$, el marco dual canónico $\left\{S_{\mathcal{F}}^{-1} f_{k}\right\}_{k \in I}$ es el de norma mínima, esto es, para todo $x \in \mathcal{H}$,

$$
\sum_{k \in I}\left|\left\langle x, S^{-1} f_{k}\right\rangle\right|^{2} \leq \sum_{k \in I}\left|\left\langle x, g_{k}\right\rangle\right|^{2}
$$

\subsection{Marcos de fusión}

Sea $\mathcal{W}=\left\{W_{i}\right\}_{i \in I}$ una sucesión de subespacios cerrados no triviales de un espacio de Hilbert $\mathcal{H}$ y sea $w=\left\{w_{i}\right\}_{i \in I} \in \ell_{+}^{\infty}(I)$ una familia de constantes positivas denominadas pesos. Denotaremos por $\mathcal{W}_{w}$ a la familia de subespacios $\mathcal{W}=\left\{W_{i}\right\}_{i \in I}$ con los pesos $w$.

Definición 1.4.1. $\mathcal{W}_{w}$ es un marco de fusión para $\mathcal{H}$ si existen constantes positivas $A, B$ tales que

$$
A\|x\|^{2} \leq \sum_{i \in I} w_{i}^{2}\left\|P_{W_{i}} x\right\|^{2} \leq B\|x\|^{2} \text { para todo } x \in \mathcal{H}
$$

Las cotas óptimas para (1.12) las denotamos por $A_{\mathcal{W}_{w}}$ y $B_{\mathcal{W}_{w}}$.

Llamamos base ortonormal de subespacios a un marco de fusión $\mathcal{W}_{w}$ que verifica que

- $w=\mathbf{e}$, siendo e el vector de $\ell_{+}^{\infty}(I)$ que tiene 1 en cada entrada.

- $W_{i} \perp W_{j}$ para todo $i \neq j$.

La definición 1.4.1 puede considerarse respecto a un subespacio cerrado $\mathcal{S}$ de $\mathcal{H}$ y en tal caso decimos que $\mathcal{W}_{w}$ es un marco de fusión para $\mathcal{S}$. En particular, decimos que $\mathcal{W}_{w}$ es una sucesión marco de fusión si el subespacio considerado es $\mathcal{S}=\overline{\operatorname{span}}\left\{W_{i}\right\}_{i=1}^{\infty}$.

Así como se hace para marcos de vectores, se pueden considerar los operadores de síntesis, análisis y de marco para marcos de fusión. Para esto, deben considerarse espacios apropiados que dependen del marco en cuestión. Para un marco de fusión $\mathcal{W}_{w}$ se define el espacio de Hilbert $\mathcal{K}_{\mathcal{W}}:=\bigoplus_{i \in I} W_{i}$ dotado con la norma $\ell^{2}$ :

$$
\|g\|^{2}=\sum_{i \in I}\left\|g_{i}\right\|^{2}, g=\left(g_{i}\right)_{i \in I} \in \mathcal{K}_{\mathcal{W}}
$$

Ahora, podemos definir el operador de síntesis: $T_{\mathcal{W}_{w}}: \mathcal{K}_{\mathcal{W}} \rightarrow \mathcal{H}$ dado por

$$
T_{\mathcal{W}_{w}}(g)=\sum_{i \in I} w_{i} g_{i}, \text { para } g=\left(g_{i}\right)_{i \in I} \in \mathcal{K}_{\mathcal{W}}
$$

Por otro lado, $T_{\mathcal{W}_{w}}^{*} \in L\left(\mathcal{H}, \mathcal{K}_{\mathcal{W}}\right)$ es el operador de análisis de $\mathcal{W}_{w}$ y está definido por:

$$
T_{\mathcal{W}_{w}}^{*}(f)=\left\{w_{i} P_{W_{i}} f\right\}_{i \in I} \text {, para todo } f \in \mathcal{H}
$$


Por último, el operador de marco: $S_{\mathcal{W}_{w}}=T_{\mathcal{W}_{w}} T_{\mathcal{W}_{w}}^{*} \in L(\mathcal{H})^{+}$, satisface

$$
S_{\mathcal{W}_{w}} f=\sum_{i \in I} w_{i}^{2} P_{W_{i}} f_{i} \text { para } f \in \mathcal{H}
$$

Observación 1.4.2. Los marcos de fusión resultan ser una de las generalizaciones más conocidas de los marcos de vectores. Esto es, todo marco $\mathcal{F}=\left\{f_{i}\right\}_{i \in I}$ para $\mathcal{H}$, se lo puede considerar como un marco de fusión $\mathcal{W}_{w}$ si tomamos los subespacios unidimensionales $W_{i}=\operatorname{span}\left\{f_{i}\right\}$ y los pesos $w_{i}=\left\|f_{i}\right\|$.

El siguiente resultado muestra que a partir de sucesiones marco, con los pesos apropiados, se puede obtener un marco para el espacio de Hilbert. Asimismo, si se cuenta con un marco de fusión, es posible obtener un marco considerando bases ortonormales de cada subespacio.

Teorema 1.4.3. Sea $\mathcal{W}=\left\{W_{i}\right\}_{i \in I}$ una sucesión de subespacios cerrados de $\mathcal{H}$ y sea $w \in \ell_{+}^{\infty}(I)$. Para cada $i \in I$, sea $\mathcal{G}_{i}=\left\{f_{i j}\right\}_{j \in J_{i}}$ un marco para $W_{i}$. Supongamos que

$$
0<A=\inf _{i \in I} A_{\mathcal{G}_{i}} \quad y \quad B=\sup _{i \in I} B_{\mathcal{G}_{i}}<\infty .
$$

Sea $\mathcal{E}_{i}=\left\{e_{i k}\right\}_{k \in K_{i}}$ una base ortonormal para cada $W_{i}$. Entonces son equivalentes:

1. $\mathcal{F}=\left\{w_{i} f_{i j}\right\}_{i \in I, j \in J_{i}}=\left\{w_{i} \mathcal{G}_{i}\right\}_{i \in I}$ es un marco para $\mathcal{H}$.

2. $\mathcal{E}=\left\{w_{i} e_{i k}\right\}_{i \in I, k \in K_{i}}=\left\{w_{i} \mathcal{E}_{i}\right\}_{i \in I}$ es un marco para $\mathcal{H}$.

3. $\mathcal{W}_{w}=\left(w_{i}, W_{i}\right)_{i \in I}$ es un marco de fusión para $\mathcal{H}$.

En este caso, las cotas de marco para $\mathcal{W}_{w}, \mathcal{F}$ y $\mathcal{E}$ satisfacen las desigualdades

$$
\frac{A_{\mathcal{F}}}{B} \leq A_{\mathcal{W}_{w}}=A_{\mathcal{E}} \leq \frac{A_{\mathcal{F}}}{A} \quad y \quad \frac{B_{\mathcal{F}}}{B} \leq B_{\mathcal{E}}=B_{\mathcal{W}_{w}} \leq \frac{B_{\mathcal{F}}}{A}
$$

\subsection{Matrices y autovalores}

Sea $\mathcal{M}_{k, d}(\mathbb{C})$ el espacio de matrices complejas de dimensión $k \times d$ y $\mathcal{M}_{d}(\mathbb{C})$ en el caso que sean matrices cuadradas con $k=d$. En $\mathcal{M}_{d}(\mathbb{C})$ consideramos dos subespacios: el subespacio real de matrices autoadjuntas, que denotamos por $\mathcal{H}(d)$, y las matrices definidas positivas que denotamos por $\mathcal{P}(d)$. Por otra parte, $\mathcal{U}(d)$ denota el grupo de matrices unitarias de $\mathcal{M}_{d}(\mathbb{C})$.

Dado un vector $x \in \mathbb{C}^{d}$ denotamos por $D_{x}$ a la matriz diagonal de $\mathcal{M}_{d}(\mathbb{C})$ cuya diagonal está dada por $x$. Si $x, y \in \mathbb{C}^{d}$ denotamos por $x \otimes y \in \mathcal{M}_{d}(\mathbb{C})$ a la matriz de rango 1 dada por $(x \otimes y) z=\langle z, y\rangle x$, para $z \in \mathbb{C}^{d}$.

Dado $x=\left(x_{i}\right)_{i \in \mathbb{I}_{d}} \in \mathbb{R}^{d}$, siendo $\mathbb{I}_{d}=\{1, \ldots, d\}$, denotamos por $x^{\downarrow}=\left(x_{i}^{\downarrow}\right)_{i \in \mathbb{I}_{d}}$ al vector que se obtiene ordenando sus entradas en orden no creciente (análogamente, $x^{\uparrow}$ denota en orden no decreciente). Los vectores de $\mathbb{R}^{d}$ ordenados en forma no creciente 
los denotamos por $\left(\mathbb{R}^{d}\right)^{\downarrow}=\left\{x^{\downarrow}: x \in \mathbb{R}^{d}\right\}$ y por $\left(\mathbb{R}_{\geq 0}^{d}\right)^{\downarrow}=\left\{x^{\downarrow}: x \in \mathbb{R}_{\geq 0}^{d}\right\}$ si tomamos entradas no negativas. Análogamente, se definen $\left(\mathbb{R}^{d}\right)^{\uparrow}$ y $\left(\mathbb{R}_{\geq 0}^{d}\right)^{\uparrow}$.

Dada una matriz $A \in \mathcal{M}_{d}(\mathbb{C})$ denotamos por

$$
\lambda(A)=\lambda(A)^{\downarrow}=\left(\lambda_{i}(A)\right)_{i \in \mathbb{I}_{d}} \in\left(\mathbb{R}^{d}\right)^{\downarrow}
$$

a los autovalores de $A$ contando multiplicidades y ordenados en orden no creciente. Dada $B \in \mathcal{M}_{d}(\mathbb{C})$ denotaremos por $s(B)=\lambda(|B|)$ a los valores singulares de $B$, es decir, los autovalores de la matriz $|B|=\left(B^{*} B\right)^{1 / 2} \in \mathcal{H}(d)$ contando multiplicidades y ordenados de manera no creciente. Asociados a estos autovalores se puede considerar la llamada descomposición en valores singulares, esto es, existen $U, V \in \mathcal{U}(d)$ tales que

$$
B=U^{*} D_{s(B)} V .
$$

La órbita unitaria de un vector $x=\left(x_{i}\right)_{i \in \mathbb{I}_{d}} \in \mathbb{R}^{d}$ la denotamos por

$$
\mathcal{O}_{x}=\left\{G \in \mathcal{M}_{d}(\mathbb{C}): \lambda(G)=x\right\}=\left\{V^{*} D_{x} V: V \in \mathcal{U}(d)\right\}
$$

Dado un conjunto de matrices $\mathcal{S}=\mathcal{S}^{*}=\left\{A \in \mathcal{M}_{d}(\mathbb{C}): A^{*} \in \mathcal{S}\right\} \subset \mathcal{M}_{d}(\mathbb{C})$, el conmutante de $\mathcal{S}$ es la $*$-subálgebra unital de $\mathcal{M}_{d}(\mathbb{C})$ dada por

$$
\mathcal{S}^{\prime}=\left\{C \in \mathcal{M}_{d}(\mathbb{C}):[C, D]=0 \text { para todo } D \in \mathcal{S}\right\} \subset \mathcal{M}_{d}(\mathbb{C})
$$

\subsubsection{Mayorización}

La mayorización y log-mayorización entre vectores de $\mathbb{R}^{d}$ tienen un rol fundamental en el análisis matricial. A pesar de no ser un orden total, la mayorización permite obtener muchas de las desigualdades matriciales conocidas por medio de la comparación de los autovalores y valores singulares. Para teoría de la mayorización recomendamos el libro de G. Hardy, J. Littlewood y G. Polya [67] (quienes introdujeron esta noción), el libro de A. Marshall y I. Olkin [87] y el artículo de T. Ando $[2]$.

Definición 1.5.1. Sean $x, y \in \mathbb{R}^{d}$. Decimos que $x$ está submayorizado por $y$ (denotado por $x \prec_{w} y$ ), si

$$
\sum_{i=1}^{j} x_{i}^{\downarrow} \leq \sum_{i=1}^{j} y_{i}^{\downarrow} \quad \text { para todo } \quad 1 \leq j \leq d .
$$

Si $x \prec_{w} y$ y $\operatorname{tr} x=\sum_{i=1}^{d} x_{i}=\sum_{i=1}^{d} y_{i}=\operatorname{tr} y$, entonces $x$ está mayorizado por $y, \mathrm{y}$ lo denotamos $x \prec y$.

Observación 1.5.2. Dados $x, y \in \mathbb{R}^{d}$, denotamos por $x \leqslant y$, si tenemos que $x_{i} \leq y_{i}$ para todo $i \in \mathbb{I}_{d}$. Las siguientes propiedades son ejercicios clásicos de mayorización: 
1. $x \leqslant y \Longrightarrow x^{\downarrow} \leqslant y^{\downarrow} \Longrightarrow x \prec_{w} y$.

2. $x \prec y \Longrightarrow|x| \prec_{w}|y|$, donde $|x|=\left(\left|x_{i}\right|\right)_{i \in \mathbb{I}_{d}} \in \mathbb{R}_{\geq 0}^{d}$.

3. $x \prec y,|x|^{\downarrow}=|y|^{\downarrow} \Longrightarrow x^{\downarrow}=y^{\downarrow}$.

Lo que puede considerarse como una versión multiplicativa de la mayorización es la log-mayorización: dados $x, y \in \mathbb{R}_{\geq 0}^{d}$, decimos que $x$ está log-mayorizado por $y$, denotándolo por $x \prec_{\text {log }} y$, si tenemos que

$$
\prod_{i=1}^{k} x_{i}^{\downarrow} \leq \prod_{i=1}^{k} y_{i}^{\downarrow} \text { para todo } k \in \mathbb{I}_{d-1} \quad \text { y } \quad \prod_{i=1}^{d} x_{i}^{\downarrow} \leq \prod_{i=1}^{d} y_{i}^{\downarrow} .
$$

La mayorización y log-mayorización tienen propiedades interesantes respecto a la traza para funciones convexas.

Proposición 1.5.3. [8] Sean $x, y \in \mathbb{R}^{d}$ y $f: \mathbb{R} \rightarrow \mathbb{R}$ una función convexa.

1. Si $x \prec y$, entonces $\operatorname{tr}(f(x)):=\sum_{i=1}^{d} f\left(x_{i}\right) \leq \sum_{i=1}^{d} f\left(y_{i}\right)=\operatorname{tr}(f(y))$.

2. Si $x \prec_{w}$ y y $f$ es creciente, $\operatorname{tr}(f(x)) \leq \operatorname{tr}(f(y))$.

3. Si $x \prec_{\log } y$ entonces $x \prec_{w} y$, por lo que

4. Si $x \prec_{\text {log }}$ y y $f$ es creciente se tiene que $\operatorname{tr}(f(x)) \leq \operatorname{tr}(f(y))$.

5. Si $f$ es una función estrictamente convexa tal que $\operatorname{tr}(f(x))=\operatorname{tr}(f(y))$,

- $x \prec y$ implica que existe una permutación $\sigma$ tal que $y_{i}=x_{\sigma(i)}$, esto es, $x^{\downarrow}=y^{\downarrow}$.

- si $x \prec_{\log }$ y y $f: \mathbb{R}_{\geq 0} \rightarrow \mathbb{R}$ es además no decreciente, entonces existe una permutación $\sigma$ tal que $y_{i}=x_{\sigma(i)}$.

Esta noción provee condiciones para la resolución de diversos problemas matriciales. En particular, podemos mencionar el problema de Schur-Horn, que consiste en determinar la existencia de un marco con las normas de sus vectores prefijadas.

Proposición 1.5.4. Sea $B \in \mathcal{P}(d)$ con autovalores $\lambda^{\downarrow}=\left(\lambda_{i}\right)_{i=1}^{d}$ y sea $a=\left(\alpha_{i}\right)_{i=1}^{k}$ con $\alpha_{1} \geq \ldots \alpha_{k}>0$. Existe una sucesión $\mathcal{G}=\left\{g_{i}\right\}_{i=1}^{k}$ en $\mathcal{H}(d)$ con operador de marco $S_{\mathcal{G}}=B$ tal que $\left\|g_{i}\right\|^{2}=\alpha_{i}$ para todo $1 \leq i \leq k$ si y sólo si $a \prec \lambda$.

\subsubsection{Normas unitariamente invariantes}

Una norma $N(\cdot)$ en $\mathcal{M}_{d}(\mathbb{C})$ es

- unitariamente invariante si

$$
N(U A V)=N(A) \quad \text { para todo } \quad A \in \mathcal{M}_{d}(\mathbb{C}) \quad \text { y } \quad U, V \in \mathcal{U}(d),
$$


- estrictamente convexa si su restricción a las matrices diagonales es una norma estrictamente convexa en $\mathbb{C}^{d}$.

Denotaremos por $N(\cdot)$ a las normas unitariamente invariantes y nos referiremos a estas normas con la sigla NUI (NUIs en plural). Ejemplos clásicos de NUIs son:

1. la norma espectral: $\|A\|_{s p}=s_{1}(A)$,

2. las normas Ky-Fan: $\|A\|_{(k)}=\sum_{i=1}^{k} \sigma_{i}$

3. las normas- $p$ : $\|A\|_{p}=\operatorname{tr}\left(|A|^{p}\right)^{1 / p}$, para $p \geq 1$ (estrictamente convexa si $p>1$ ).

Estas normas se pueden relacionar con las llamadas funciones gauge simétricas en $\mathbb{R}^{n}$ por medio de los valores singulares.

Definición 1.5.5. Una función $\Phi$ es una función gauge simétrica en $\mathbb{R}^{n}$ si

1. $\Phi$ es una norma,

2. $\Phi(P x)=\Phi(x)$ para todo $x \in \mathbb{R}^{n}$ y toda permutación $P$.

3. $\Phi\left(a_{1} x_{1}, \ldots, a_{n} x_{n}\right)=\Phi\left(x_{1}, \ldots, x_{n}\right)$ si $a_{j}= \pm 1$.

Teorema 1.5.6. [8] Si $\Phi$ una función gauge simétrica en $\mathbb{R}^{n}$,

$$
\|\mid A\|_{\Phi}=\Phi(s(A))
$$

define una $N U I$ en $\mathcal{M}_{n}(\mathbb{R})$. Recíprocamente, si $N(\cdot)$ es una $N U I$, la función

$$
\Phi_{N}(x)=N\left(D_{x}\right)
$$

define una función gauge simétrica en $\mathbb{R}^{n}$.

Otra relación de las NUIs con los valores singulares es a través de la mayorización. El siguiente resultado ilustra muy bien este hecho (ver por ejemplo el libro de Bhatia [18]):

Teorema 1.5.7. Sean $A, B \in \mathcal{M}_{d}(\mathbb{C})$ tal que $s(A) \prec_{w} s(B)$. Entonces:

1. Para toda NUI $N(\cdot)$ en $\mathcal{M}_{d}(\mathbb{C})$ se tiene que $N(A) \leq N(B)$.

2. Si $N(\cdot)$ es una NUI estrictamente convexa en $\mathcal{M}_{d}(\mathbb{C})$ y $N(A)=N(B)$, entonces $s(A)=s(B)$. 


\subsubsection{Desigualdades de Lidskii}

En la década del '50, V. Lidskii fue el precursor de una serie de resultados vinculados a desigualdades espectrales. Fue H. Wielandt (1955) quién encontró una conexión entre el resultado original de Lidskii y las desigualdades de Ky Fan. En su resultado original de 1950, Lidskii aseguró que, si $\alpha=\left(\alpha_{i}\right)_{i=1}^{n}$ y $\beta=\left(\beta_{i}\right)_{i=1}^{n}$ son los autovalores de $A$ y $B$, los autovalores de $A+B$ se encontraban en la cápsula convexa de los vectores $\alpha+\beta_{\sigma}$, siendo $\beta_{\sigma}$ las $n$ ! permutaciones de las entradas de $\beta$. Wielandt desarrolló su conocido principio de minimax aportando una de las primeras pruebas alternativas del Teorema de Lidskii [103], entre las existentes en la literatura. De las diversas demostraciones, en palabras de Bhatia [10], "la prueba más sencilla" se debe a C. K. Li y R. Mathias [84].

Teorema 1.5.8. (Lisdkii) Sean $A, B \in \mathcal{H}(d)$ con autovalores $\lambda(A), \lambda(B) \in\left(\mathbb{R}^{d}\right)^{\downarrow}$ entonces

$$
\lambda(A)^{\uparrow}+\lambda(B) \prec \lambda(A+B) .
$$

En [61], Gelfand y Naimark probaron una versión multiplicativa de Lidskii para valores singulares:

Teorema 1.5.9. (Gelfand, Naimark) Sean $A, B \in \mathcal{P}(d)$. Entonces

$$
\log (s(A B))-\log (s(B) \prec \log (s(A))
$$

Relacionando estos resultados se tiene el

Teorema 1.5.10. (Gelfand-Naimark-Lidskii) Sean $A, B \in \mathcal{P}(d)$. Entonces

$$
\log \left(\lambda^{\downarrow}(B)\right)+\log \left(\lambda^{\uparrow}(A)\right) \prec \log (\lambda(B A)) .
$$

Notar que, en particular,

$$
\log \left(\lambda^{\downarrow}(B)\right)-\log \left(\lambda^{\downarrow}(A)\right) \prec \log \left(\lambda\left(B A^{-1}\right)\right) \prec \log \left(\lambda^{\downarrow}(B)\right)-\log \left(\lambda^{\uparrow}(A)\right) .
$$

En la teoría de marcos, las desigualdades de Lidskii contribuyeron con los llamados problemas de diseño óptimo. En ese contexto, podemos mencionar dos problemas de perturbación estudiados en [91]: perturbación óptima de duales canónicos con restricciones, esto es, dado un marco finito $\mathcal{F}$ para un Hilbert $\mathcal{H}$ de dimensión finita, se busca calcular los marcos duales $\mathcal{G}$, con restricciones de norma sobre sus elementos; perturbación óptima por marcos equivalentes, lo que consiste en considerar los operadores inversibles $V$ próximos a la identidad, de manera que $V(\mathcal{F})$ sea óptimo con respecto a la dispersión de los autovalores de su operador de marco [91]. En el caso particular de marcos de Parseval, en un trabajo reciente aparecen las desigualdades en un problema de mejor aproximante a un marco arbitrario por medio de marcos de Parseval (en espacios de Hilbert de dimensión infinita) [33]. 


\subsubsection{Aspectos locales}

Para el estudio local de problemas que involucran espacios de matrices es natural considerar estructuras geométricas. Por ejemplo, en el trabajo [79] R. Leite, T. Richa y C. Tomei prueban con técnicas geométricas y simplécticas casos particulares del Teorema de Schur-Horn, tanto en su versión autoadjunta como en versiones menos conocidas para matrices anti-simétricas. Muchas de las estructuras definidas en ese trabajo se encuentran también en el contexto local de las desigualdades de Lidskii que estudiamos en el capítulo 5. En esta sección incluimos brevemente las nociones de geometría diferencial que serán necesarias para los resultados de ese capítulo.

Dada una variedad diferenciable $\mathcal{N}$ y $p \in \mathcal{N}$, denotamos por $\mathcal{T}_{p} \mathcal{N}$ al espacio tangente de $\mathcal{N}$ en $p$.

Definición 1.5.11. Si $\mathcal{N}$ y $\mathcal{M}$ son variedades diferenciables y $F: \mathcal{N} \rightarrow \mathcal{M}$ es una aplicación diferenciable, $F$ es una submersión en $p \in \mathcal{N}$ si su diferencial

$$
D_{p} F: \mathcal{T}_{p} \mathcal{N} \rightarrow \mathcal{T}_{F(p)} \mathcal{M}
$$

es suryectivo. Si $F$ es una submersión en cada $p \in \mathcal{N}$ entonces se dice que $F$ es una submersión.

El siguiente resultado nos asegura que toda submersión $F$ es localmente abierta alrededor de $p_{0}$.

Teorema 1.5.12. Sean $\mathcal{N}$ y $\mathcal{M}$ variedades diferenciables y $F: \mathcal{N} \rightarrow \mathcal{M}$ una aplicación diferenciable. Si $F$ es una submersión en $p_{0} \in \mathcal{N}$ entonces para todo $\varepsilon>0$, si

$$
\mathcal{N}_{\varepsilon}:=\left\{p \in \mathcal{N}: d\left(p, p_{0}\right)<\varepsilon\right\},
$$

el conjunto $F\left(\mathcal{N}_{\varepsilon}\right)$ contiene un entorno abierto de $F\left(p_{0}\right)$ en $\mathcal{M}$.

En este trabajo consideramos a $\mathcal{U}(d) \in \mathcal{M}_{d}(\mathbb{C})$ dotado con su estructura natural de variedad diferenciable. Además, consideramos la variedad producto $\mathcal{U}(d) \times \mathcal{U}(d)$ dotada con la siguiente métrica:

$$
d\left(\left(U_{1}, V_{1}\right),\left(U_{2}, V_{2}\right)\right)=\operatorname{máx}\left\{\left\|U_{1}-U_{2}\right\|,\left\|V_{1}-V_{2}\right\|\right\},
$$

siendo $\|\cdot\|$ la norma espectral usual.

Es sabido que se puede identificar al espacio tangente en la identidad $\mathcal{T}_{I} \mathcal{U}(d)$ con el conjunto de las matrices anti-hermitianas $i \cdot \mathcal{H}(d)$ por medio de la aplicación exponencial

$$
\mathcal{H}(d) \ni i \cdot X \mapsto \exp (X),
$$

ya que la curva $\gamma(t)=\exp (t X) \in \mathcal{U}(d)$ verifica que $\gamma^{\prime}(0)=X \in i \cdot \mathcal{H}(d)$.

Dados $A, B \in \mathcal{H}(d)$, sea

$$
\mathcal{O}_{A}=\left\{V^{*} A V: V \in \mathcal{U}(d)\right\}
$$


la órbita unitaria de $A$. Si consideramos la función suave $\mathcal{C}_{A}: \mathcal{U}(d) \rightarrow \mathcal{O}_{A}$, definida por $\mathcal{C}_{A}(U)=U^{*} A U$ entonces

$$
D_{I} \mathcal{C}_{A}(X)=[X, A] \in \mathcal{H}(d) \quad \text { para } \quad X \in i \cdot \mathcal{H}(d)
$$

donde $[B, A]:=B A-A B$. 



\section{Capítulo 2}

\section{Marcos y sucesiones de Riesz}

En la teoría de marcos, se habla de exceso cuando se hace referencia a la capacidad de un marco para poder prescindir de algún elemento y que esto no implique que la sucesión pierda su esencia de marco. Aquellos marcos que dejan de serlo si alguno de sus elementos es removido se los llama exactos. Esta clase de marcos está fuertemente relacionada con las conocidas bases de Riesz.

Entre los antecedentes destacados de las bases de Riesz en el contexto de espacios de Banach, se decía que una base $\left\{f_{k}\right\}_{k=1}^{\infty}$ era Besseliana si la convergencia de $\sum_{k=1}^{\infty} c_{k} f_{k}$ implica $\sum_{k=1}^{\infty}\left|c_{k}\right|^{2}<\infty$. Por otro lado, se decía que una base $\left\{f_{k}\right\}_{k=1}^{\infty}$ era Hilbertiana si $\sum_{k=1}^{\infty}\left|c_{k}\right|^{2}<\infty$ implica la convergencia de $\sum_{k=1}^{\infty} c_{k} f_{k}$. Las sucesiones que fueran simultáneamente Besselianas y Hilbertianas son las que actualmente llamamos bases de Riesz [96].

Uno de los puntos fuertes de las bases de Riesz radica en su incondicionalidad como bases, lo que resulta de gran utilidad en las aplicaciones, por ejemplo, las hace óptimas para la compresión de señales. Como suele suceder, quizás como producto de la complejidad del problema a resolver, las sucesiones que se toman no son completas, lo que lleva a considerar las llamadas sucesiones de Riesz. Christensen muestra en [34] que los marcos exactos resultan ser sucesiones de Riesz generadoras.

Dentro de la gran cantidad de problemas abstractos que se consideran vinculados a los marcos, podemos encontrar el estudio de los subconjuntos de un marco, con especial atención en sus propiedades de independencia lineal y su capacidad para generar. Por ejemplo, se estudia a las sucesiones de Riesz como partición de marcos en conjuntos linealmente independientes. Un problema relacionado a este enfoque es el problema de Kadison-Singer para marcos [20].

Los primeros resultados de este capítulo tratan sobre caracterizaciones de sucesiones marco y sucesiones de Riesz por medio de desigualdades de operadores. Por medio de una caracterización del gap entre subespacios cerrados en términos del mínimo de una desigualdad de proyecciones, indicamos cómo tienen que ser las subfamilias de un marco para $\mathcal{H}$ para que éstas, a su vez, sean también marcos. Por último, relacionando nuestras caracterizaciones con resultados recientes de la literatura, exhibimos dos aplicaciones a marcos de Parseval. 


\subsection{Sucesiones de Riesz}

En esta primer sección examinamos las caracterizaciones clásicas relacionadas con marcos y sucesiones de Riesz. Para eso, comenzamos de forma general considerando bases ortonormales que relacionaremos con las bases de Riesz. Luego de la definición de sucesiones de Riesz, notamos que la noción alternativa de sucesiones de RieszFischer permite distinguir qué características estan vinculadas a cada una de las cotas de una sucesión de Riesz.

Dada una base ortonormal de un espacio de Hilbert separable, podemos caracterizar todas las otras a través de un operador unitario. Es decir, si $\left\{e_{k}\right\}_{k=1}^{\infty}$ es una base ortonormal para $\mathcal{H}$, entonces las bases ortonormales para $\mathcal{H}$ son precisamente los conjuntos $\left\{U e_{k}\right\}_{k=1}^{\infty}$, siendo $U \in L(\mathcal{H})$ un operador unitario. Esto se puede relacionar con la definición de bases de Riesz, en la que también se consideran perturbaciones de bases ortonormales, con la diferencia que el operador de perturbación es inversible.

Definición 2.1.1. Una base de Riesz para $\mathcal{H}$ es una familia $\left\{U e_{k}\right\}_{k=1}^{\infty}$ donde $\left\{e_{k}\right\}_{k=1}^{\infty}$ es una base ortonormal para $\mathcal{H}$ y $U \in L(\mathcal{H})$ un operador inversible.

A las bases de Riesz se las puede caracterizar a través de la siguiente desigualdad: una sucesión $\left\{f_{i}\right\}_{i \in I}$ completa para $\mathcal{H}$ es una base de Riesz en $\mathcal{H}$ si existen $a, b>0$ tales que

$$
a \sum_{i \in J}\left|c_{i}\right|^{2} \leq\left\|\sum_{i \in J} c_{i} f_{i}\right\|^{2} \leq b \sum_{i \in J}\left|c_{i}\right|^{2} .
$$

Esta desigualdad lleva a definir las sucesiones de Riesz:

Definición 2.1.2. Una sucesión $\left\{f_{i}\right\}_{i \in I}$ es una sucesión de Riesz para $\mathcal{H}$ si existen $a, b>0$ tales que

$$
a \sum_{i \in J}\left|c_{i}\right|^{2} \leq\left\|\sum_{i \in J} c_{i} f_{i}\right\|^{2} \leq b \sum_{i \in J}\left|c_{i}\right|^{2} .
$$

Es claro que una base de Riesz es un marco y que toda subfamilia de una base de Riesz resulta una sucesión de Riesz. El siguiente es un resultado conocido que muestra relaciones entre sucesiones de Riesz, bases y marcos.

Teorema 2.1.3. Sea $\left\{f_{k}\right\}_{k=1}^{\infty}$ un marco para $\mathcal{H}$. Entonces son equivalentes:

- $\left\{f_{k}\right\}_{k=1}^{\infty}$ es una base de $\mathcal{H}$.

- $\left\{f_{k}\right\}_{k=1}^{\infty}$ es una sucesión de Riesz completa para $\mathcal{H}$.

- $\left\{f_{k}\right\}_{k=1}^{\infty}$ es un marco exacto.

- $\left\{f_{k}\right\}_{k=1}^{\infty}$ es minimal i.e. $f_{j} \notin \operatorname{span}\left\{f_{k}\right\}_{k \neq j}$ para todo $j \in \mathbb{N}$.

- $\left\{f_{k}\right\}_{k=1}^{\infty}$ es $\omega$-independente i.e. si $\sum_{k=1}^{\infty} c_{k} f_{k}=0$ para algún $\left\{c_{k}\right\}_{k=1}^{\infty} \in \ell^{2}(\mathbb{N})$, entonces $c_{k}=0$ para todo $k \in \mathbb{N}$. 
En [27] los autores estudiaron aquellas sucesiones que admiten la cota inferior en (2.2) y denominaron a estas sucesiones como sucesiones de Riesz-Fischer. Estos resultados permiten identificar algunas de las propiedades que provienen de la existencia de la cota inferior de las sucesiones de Riesz. Notar las diferencias con el Teorema 2.1.3.

Teorema 2.1.4. Sea $\left\{f_{k}\right\}_{k=1}^{\infty}$ una sucesión para $\mathcal{H}$. Si se consideran los siguientes enunciados,

1. $\left\{f_{k}\right\}_{k=1}^{\infty}$ es una sucesión de Riesz-Fischer completa,

2. $\left\{f_{k}\right\}_{k=1}^{\infty}$ es minimal y satisface la cota inferior de marco,

3. $\left\{f_{k}\right\}_{k=1}^{\infty}$ es $\omega$-independente y satisface la cota inferior de marco,

entonces se tiene que 1. $\Rightarrow$ 2. $\Rightarrow 3$.

Es posible caracterizar a aquellas sucesiones que posean cota inferior de Riesz determinando la existencia de una sucesión de Bessel que cumpla una condición de ortogonalidad.

Proposición 2.1.5. Sea $\left\{f_{i}\right\}_{i=1}^{\infty}$ una sucesión de un espacio de Hilbert $\mathcal{H}$. Si existe una sucesión $\left\{g_{i}\right\}_{i=1}^{\infty}$ de Bessel tal que $\left\langle f_{i}, g_{j}\right\rangle=\delta_{i, j}$ (delta de Kronecker) entonces $\left\{f_{i}\right\}_{i=1}^{\infty}$ es una sucesión de Riesz-Fischer.

El siguiente ejemplo muestra una aplicación de la proposición que permite asegurar la existencia de la cota inferior de Riesz de una sucesión dada.

Ejemplo 2.1.6. Sea $\left\{e_{i}\right\}_{i=1}^{\infty}$ una base ortonormal para $\mathcal{H}$ y $\left\{f_{k}\right\}_{k=1}^{\infty}$ una sucesión dada por

$$
f_{k}= \begin{cases}\sum_{i=1}^{k}(-1)^{i} e_{i} & \text { si } k \text { es par } \\ \sum_{i=1}^{k}(-1)^{i+1} e_{i} & \text { si } k \text { es impar }\end{cases}
$$

Si $\left\{g_{k}\right\}_{k=1}^{\infty}=\left\{e_{k}+e_{k+1}\right\}_{k=1}^{\infty}$, es fácil de ver que es una sucesión de Bessel que verifica que $\left\langle f_{i}, g_{j}\right\rangle=\delta_{i, j}$. Por la proposición anterior, $\left\{f_{k}\right\}_{k=1}^{\infty}$ es una sucesión de RieszFischer.

\subsubsection{Sucesiones de Riesz y sucesiones marco}

Un evento que fortaleció la relación entre marcos y sucesiones de Riesz fue la aparición de la conocida conjetura de Feichtinger en 2002: si $\mathcal{F}=\left\{f_{i}\right\}_{i \in I}$ es un marco tal que ínf $\operatorname{i\in \mathbb {N}}_{i}\left\|f_{i}\right\|>0, \mathcal{F}$ se puede particionar en una unión finita de sucesiones de Riesz. Esta conjetura resultó ser equivalente a una más antigua, la conjetura de Kadison-Singer de 1959 (Casazza y Tremain, 2005). Con el transcurso de los años, la conjetura de Feichtinger fue relacionada con otros problemas hasta que fue confirmada en 2013, por Marcus, Spielman y Srivastava. 
El sentido de la dualidad entre los marcos y las sucesiones de Riesz se puede apreciar en términos de cotas de operadores. La condición de ser marco refiere a la acotación superior e inferior del operador de síntesis, mientras que la condición de sucesión de Riesz equivale a acotaciones del operador de análisis.

Concluimos la sección con un resultado que relaciona sucesiones de Riesz y sucesiones marco con desigualdades de los operadores grammiano y de marco, respectivamente. Este resultado anticipa las nociones que trabajaremos en este capítulo y las técnicas que utilizaremos.

Proposición 2.1.7. Sea $\mathcal{F}=\left\{f_{i}\right\}_{i \in I}$ una sucesión de Bessel en un espacio de Hilbert $\mathcal{H}$, con operador de sintesis $T_{\mathcal{F}}$, operador grammiano $G_{\mathcal{F}}=T_{\mathcal{F}}^{*} T_{\mathcal{F}}$ y operador de marco $S_{\mathcal{F}}$. Entonces,

1. $\mathcal{F}=\left\{f_{i}\right\}_{i \in I}$ es una sucesión de Riesz si y sólo si existe $a>0$ tal que

$$
G_{\mathcal{F}}=T_{\mathcal{F}}^{*} T_{\mathcal{F}} \geq a I d_{\ell^{2}(I)}
$$

2. $\mathcal{F}=\left\{f_{i}\right\}_{i \in I}$ es una sucesión marco si y sólo si existe $a>0$ tal que

$$
S_{\mathcal{F}}=T_{\mathcal{F}} T_{\mathcal{F}}^{*} \geq a P_{N\left(T_{\mathcal{F}}^{*}\right)^{\perp}}
$$

Demostración. Supongamos que $\mathcal{F}=\left\{f_{i}\right\}_{i \in I}$ es una sucesión de Riesz en $\mathcal{H}$, con operador de síntesis $T$. Entonces, existen $a, b>0$ tales que se verifica (2.2) para cada $\left\{c_{i}\right\}_{i \in I} \in \ell^{2}(I)$ finito. Como $T_{\mathcal{F}}$ es acotado, es fácil ver que (2.2) vale para todo $\left\{c_{i}\right\}_{i \in I}$ en $\ell^{2}(I)$. Si $\left\{c_{i}\right\}_{i \in I} \in \ell^{2}(I)$ entonces

$$
\left\langle T_{\mathcal{F}}^{*} T_{\mathcal{F}}\left\{c_{i}\right\}_{i \in I},\left\{c_{i}\right\}_{i \in I}\right\rangle=\left\|T_{\mathcal{F}}\left\{c_{i}\right\}_{i \in I}\right\|^{2} \geq a\left\|\left\{c_{i}\right\}_{i \in I}\right\|^{2}=a\left\langle\left\{c_{i}\right\}_{i \in I},\left\{c_{i}\right\}_{i \in I}\right\rangle .
$$

La recíproca se prueba también de esta desigualdad.

Por otro lado, si $\left\{f_{i}\right\}_{i \in I}$ es una sucesión marco entonces $T_{\mathcal{F}}$ es un operador con rango cerrado (simplemente por la definición de marco). Luego, como hicimos más arriba, las desigualdades de marco en $R\left(T_{\mathcal{F}}\right)=N\left(T_{\mathcal{F}}^{*}\right)^{\perp}$ implican que $T_{\mathcal{F}} T_{\mathcal{F}}^{*} \geq a P_{N\left(T_{\mathcal{F}}^{*}\right)^{\perp}}$. Un razonamiento similar se puede aplicar para la prueba de la recíproca.

\subsection{Marcos y gap entre subespacios}

En esta sección recorremos distintas condiciones que relacionan desigualdades de proyecciones ortogonales con el gap entre ciertos subespacios. Estas equivalencias dan lugar a una caracterización de las subfamilias de un marco que también sean marco. Finalmente, mostramos aplicaciones al caso particular de marcos de Parseval.

En la primera de estas desigualdades de proyecciones notamos que se puede calcular el gap entre subespacios cerrados como el mínimo de una desigualdad de proyecciones. 
Proposición 2.2.1. Sean $\mathcal{M}, \mathcal{N}$ subespacios cerrados.

$$
\delta^{2}(\mathcal{M}, \mathcal{N})=\min \left\{\lambda: 0 \leq \lambda \leq 1, P_{\mathcal{M}} P_{\mathcal{N}}^{\perp} P_{\mathcal{M}} \leq \lambda P_{\mathcal{M}}\right\}
$$

Demostración. Para la prueba, consideremos $P=P_{\mathcal{M}}$ y $Q=P_{\mathcal{N}}$.

Sea $0 \leq \lambda \leq 1$ tal que $P Q^{\perp} P \leq \lambda P$. De (1.2.2) tenemos que

$$
\left\|P Q^{\perp} P\right\|^{2}=\left\|\left(Q^{\perp} P\right)^{*}\left(Q^{\perp} P\right)\right\|=\left\|Q^{\perp} P\right\|^{2}=\delta(\mathcal{M}, \mathcal{N})^{2} .
$$

Como por $P Q^{\perp} P$ es autoadjunto,

$$
P Q^{\perp} P \leq \delta(\mathcal{M}, \mathcal{N})^{2} I
$$

Conjugando con $P$ vemos que

$$
P Q^{\perp} P \leq \delta(\mathcal{M}, \mathcal{N})^{2} P
$$

Por otro lado, si $x \in \mathcal{H},\|x\|=1$, tenemos que

$$
\begin{aligned}
\left\|Q^{\perp} P x\right\|^{2} & =\left\langle Q^{\perp} P x, Q^{\perp} P x\right\rangle \\
& =\left\langle P Q^{\perp} P x, x\right\rangle \leq\langle\lambda P x, x\rangle=\lambda\|P x\|^{2} \leq \lambda .
\end{aligned}
$$

Tomando supremo sobre $\|x\|=1$, como $\delta(\mathcal{M}, \mathcal{N})^{2}=\left\|Q^{\perp} P\right\|^{2}$, tenemos que

$$
\delta(\mathcal{M}, \mathcal{N})^{2} \leq \lambda
$$

De este resultado obtenemos una serie de equivalencias:

Corolario 2.2.2. Sea $\mathcal{M}, \mathcal{N}$ subespacios cerrados de $\mathcal{H}$. Son equivalentes:

1. existe $a>0$ tal que $P_{\mathcal{M}} P_{\mathcal{N}} P_{\mathcal{M}} \geq a P_{\mathcal{M}}$.

2. $\delta(\mathcal{M}, \mathcal{N})<1$.

3. $c_{0}\left(\mathcal{M}, \mathcal{N}^{\perp}\right)<1$.

4. $P_{\mathcal{M}} P_{\mathcal{N}}$ tiene rango cerrado y $R\left(P_{\mathcal{M}} P_{\mathcal{N}}\right)=\mathcal{M}$.

5. existe $X \in L(\mathcal{H})$ con $\|X\|=\frac{1}{a}$ tal que $P_{\mathcal{M}}=P_{\mathcal{M}} P_{\mathcal{N}} X$.

Demostración. Las equivalencia entre 1. 2. y 3. se deduce del resultado anterior y de los preliminares de gap. Por otro lado, 1. 4. y 5. son consecuencia del Teorema de Douglas (Teorema 1.2.5). 
Notemos que este resultado, en un Hilbert de dimensión finita, permite probar el resultado conocido que indica que

$$
\text { si }\left\|P_{\mathcal{M}}-P_{\mathcal{N}}\right\|<1 \text { entonces } \operatorname{dim}(\mathcal{M})=\operatorname{dim}(\mathcal{N}) \text {. }
$$

Si $\left\|P_{\mathcal{M}}-P_{\mathcal{N}}\right\|=\widehat{\delta}(\mathcal{M}, \mathcal{N})<1$ entonces $\delta(\mathcal{M}, \mathcal{N})<1$ y $\delta(\mathcal{N}, \mathcal{M})<1$. Por el corolario esto implica que

$$
R\left(P_{\mathcal{N}} P_{\mathcal{M}}\right)=\mathcal{N} \text { y } R\left(P_{\mathcal{M}} P_{\mathcal{N}}\right)=\mathcal{M}
$$

con lo cual, $\operatorname{dim}(\mathcal{N}) \leq \operatorname{dim}(\mathcal{M})$ y $\operatorname{dim}(\mathcal{M}) \leq \operatorname{dim}(\mathcal{N})$

La siguiente equivalencia entre desigualdades de producto de proyecciones se vale de la caracterización del gap del comienzo de esta sección y es de utilidad para varios resultados de este capítulo.

Proposición 2.2.3. Sea $\mathcal{H}$ un espacio de Hilbert complejo separable y sean $P$ y $Q$ proyecciones ortogonales. Entonces, son equivalentes:

i. Existe $0<a \leq 1$ tal que $P Q P \geq a P$.

ii. Existe $0<a \leq 1$ tal que $Q^{\perp} P^{\perp} Q^{\perp} \geq a Q^{\perp}$.

Más aún, si $\delta^{2}(R(P), R(Q))<1$, se puede tomar $a=1-\delta^{2}(R(P), R(Q))$.

Demostración. Supongamos que $P Q P \geq a P$. Considerando que $Q^{\perp}=I-Q$, tenemos que

$$
P Q^{\perp} P \leq(1-a) P
$$

Por el teorema anterior,

$$
\delta^{2}(R(P), R(Q)) \leq 1-a .
$$

Por la Proposición 1.2.2,

$$
\delta(R(P), R(Q))=\delta\left(R(Q)^{\perp}, R(P)^{\perp}\right) .
$$

Luego, aplicando de nuevo la Proposición 2.2.1 tenemos que

$$
\begin{aligned}
Q^{\perp} P^{\perp} Q^{\perp} & \leq \delta\left(R(P)^{\perp}, R(Q)^{\perp}\right) Q^{\perp} \\
& \leq(1-a) Q^{\perp} .
\end{aligned}
$$

Reescribiendo esta desigualdad tenemos $i i$. La prueba de la recíproca se desarrolla de la misma forma.

Teorema 2.2.4. Sea $\mathcal{F}=\left\{f_{i}\right\}_{i \in I}$ un marco para $\mathcal{H}$ y $\sigma \subset I$, entonces

$$
\mathcal{F}_{\sigma}=\left\{f_{i}\right\}_{i \in \sigma} \text { es marco para } \mathcal{H} \text { si y sólo si } \delta^{2}\left(R\left(T_{\mathcal{F}}^{*}\right), R\left(P_{\sigma}\right)\right)<1 \text {. }
$$


Demostración. Supongamos que $\delta^{2}\left(R\left(T_{\mathcal{F}}^{*}\right), R\left(P_{\sigma}\right)\right)<1$. Por la Proposición 2.2.3, para $\alpha=1-\delta^{2}\left(R\left(T_{\mathcal{F}}^{*}\right), R\left(P_{\sigma}\right)\right)$,

$$
P_{R\left(T_{\mathcal{F}}^{*}\right)} P_{\sigma} P_{R\left(T_{\mathcal{F}}^{*}\right)} \geq \alpha P_{R\left(T_{\mathcal{F}}^{*}\right)}
$$

Notar que $P_{R\left(T_{\mathcal{F}}^{*}\right)}=T_{\mathcal{F}}^{*} S_{\mathcal{F}}^{-1} T_{\mathcal{F}}$, entonces

$$
T_{\mathcal{F}}^{*} S_{\mathcal{F}}^{-1} T_{\mathcal{F}} P_{\sigma} T_{\mathcal{F}}^{*} S_{\mathcal{F}}^{-1} T_{\mathcal{F}} \geq \alpha T_{\mathcal{F}}^{*} S_{\mathcal{F}}^{-1} T_{\mathcal{F}}
$$

Conjugando con $T_{\mathcal{F}}$ y $T_{\mathcal{F}}^{*}$ y por definición del operador de marco $S_{\mathcal{F}}$,

$$
T_{\mathcal{F}} P_{\sigma} T_{\mathcal{F}}^{*} \geq \alpha S_{\mathcal{F}}
$$

Por Teorema 1.2.5 (Douglas), esta última desigualdad implica que

$$
\mathcal{H}=R\left(S_{\mathcal{F}}^{1 / 2}\right) \subset R\left(T_{\mathcal{F}} P_{\sigma}\right),
$$

pero $R\left(S_{\mathcal{F}}^{1 / 2}\right)=\mathcal{H}$, por lo que $T_{\mathcal{F}} P_{\sigma}$ resulta suryectiva.

Recíprocamente, supongamos que $\mathcal{F}_{\sigma}=\left\{f_{i}\right\}_{i \in \sigma}$ es marco con cota inferior $A_{\sigma}>0$. Considerando la desigualdad de operadores (1.8) para $S_{\mathcal{F}_{\sigma}}$,

$$
\left(T_{\mathcal{F}} P_{\sigma}\right)\left(T_{\mathcal{F}} P_{\sigma}\right)^{*}=T_{\mathcal{F}} P_{\sigma} T_{\mathcal{F}}^{*} \geq A_{\sigma} I
$$

Como $S_{\mathcal{F}}^{-1} \geq \frac{1}{B_{\mathcal{F}}} I$, conjugando (2.4) primero con $S_{\mathcal{F}}^{-1}$ y luego con $T_{\mathcal{F}}^{*}$ y $T_{\mathcal{F}}$ :

$$
\begin{aligned}
S_{\mathcal{F}}^{-1} T_{\mathcal{F}} P_{\sigma} T_{\mathcal{F}}^{*} S_{\mathcal{F}}^{-1} & \geq A_{\sigma} S_{\mathcal{F}}^{-2} \geq \frac{A_{\sigma}}{B_{\mathcal{F}}} S_{\mathcal{F}}^{-1} \\
T_{\mathcal{F}}^{*} S_{\mathcal{F}}^{-1} T_{\mathcal{F}} P_{\sigma} T_{\mathcal{F}}^{*} S_{\mathcal{F}}^{-1} T_{\mathcal{F}} & \geq \frac{A_{\sigma}}{B_{\mathcal{F}}} T_{\mathcal{F}}^{*} S_{\mathcal{F}}^{-1} T_{\mathcal{F}} \\
P_{R\left(T_{\mathcal{F}}^{*}\right)} P_{\sigma} P_{R\left(T_{\mathcal{F}}^{*}\right)} & \geq \frac{A_{\sigma}}{B_{\mathcal{F}}} P_{R\left(T_{\mathcal{F}}^{*}\right)} .
\end{aligned}
$$

Luego, por la Proposición 2.2.1,

$$
\delta^{2}\left(R\left(T_{\mathcal{F}}^{*}\right), R\left(P_{\sigma}\right)\right)<1-\frac{A_{\sigma}}{B_{\mathcal{F}}}<1
$$

\subsection{Aplicaciones del gap a marcos de Parseval}

En esta última sección mencionamos dos aplicaciones a marcos de Parseval a partir de resultados de las secciones previas. Recordamos que los marcos de Parseval son un caso particular de la clase de marcos ajustados donde las cotas de marco coinciden. Es un hecho conocido que la función que desempeña todo marco en términos de reconstrucción la puede llevar a cabo un marco de Parseval. En efecto, si $\mathcal{F}=\left\{f_{i}\right\}_{i \in I}$ 
es un marco, para todo $x \in \mathcal{H}$,

$$
\begin{aligned}
x & =S_{\mathcal{F}} S_{\mathcal{F}}^{-1} x=\sum_{i \in I}\left\langle x, S_{\mathcal{F}}^{-1} f_{i}\right\rangle f_{i} \\
& =S_{\mathcal{F}}^{-1 / 2}\left(\sum_{i \in I}\left\langle x, S_{\mathcal{F}}^{-1 / 2} f_{i}\right\rangle f_{i}\right)=\sum_{i \in I}\left\langle x, S_{\mathcal{F}}^{-1 / 2} f_{i}\right\rangle S_{\mathcal{F}}^{-1 / 2} f_{i} .
\end{aligned}
$$

En otras palabras, todo marco $\mathcal{F}=\left\{f_{i}\right\}_{i \in I}$ admite como perturbación al marco de Parseval $\left\{S_{\mathcal{F}}^{-1 / 2} f_{i}\right\}_{i \in I}$.

Otra aproximación de un marco a los marcos de Parseval es a través de la noción de marcos $\varepsilon$-casi Parseval [19]: un marco $\mathcal{F}=\left\{f_{i}\right\}_{i \in I}$ se dice $\varepsilon$-casi Parseval si existe $\varepsilon>0$ tal que para todo $x \in \mathcal{H}$

$$
(1-\varepsilon)\|x\|^{2} \leq \sum_{i \in I}\left|\left\langle x, f_{i}\right\rangle\right| \leq(1+\varepsilon)\|x\|^{2}
$$

Notar que si $\varepsilon=0$ tendríamos la igualdad de la definición de marco de Parseval. Por otro lado, si $\mathcal{F}$ es un marco $\varepsilon$-casi Parseval su grammiano $G_{\mathcal{F}}$ satisface que $(1-\varepsilon) I \leq G_{\mathcal{F}} \leq(1+\varepsilon) I$.

\subsubsection{Sucesiones de Riesz y sucesiones marco}

Como hemos dicho anteriormente, existen numerosos resultados que relacionan las sucesiones de Riesz con los marcos y su descomposición en subfamilias. A estas subfamilias se les suele pedir que sean linealmente independientes o que generen algún subespacio específico. En [95], Olevskii y Ulanovskii realizan una descomposición de una base ortonormal en dos subconjuntos disjuntos y aplican proyecciones ortogonales a dichos subconjuntos. Prueban que, mientras uno de los conjuntos proyectados es una sucesión marco, el otro tiene que ser una sucesión de Riesz. Por su simpleza incluimos la prueba a continuación.

Proposición 2.3.1. [95] Sea $\mathcal{U}$ una base ortonormal de $\mathcal{H}$. Supongamos que $\mathcal{U}$ es unión de dos subconjuntos disjuntos $\mathcal{V}$ y $\mathcal{W}$ y que $\mathcal{H}$ es suma directa de dos subespacios ortogonales $\mathcal{H}_{1}$ y $\mathcal{H}_{2}$, siendo $P_{j}$ las proyecciones ortogonales en $\mathcal{H}_{j}$. Las siguientes afirmaciones son equivalentes:

- $P_{1}(\mathcal{V})$ es un marco en $\mathcal{H}_{1}$.

- $P_{2}(\mathcal{W})$ es una sucesión de Riesz en $\mathcal{H}_{2}$.

Demostración. Supongamos que $P_{1}(\mathcal{V})$ es un marco en $\mathcal{H}_{1}$. Tomemos $f \in P_{2}(\mathcal{W})$ y $f_{i} \in \mathcal{H}_{i}$ tales que

$$
f=f_{1}+f_{2}=: \sum_{\omega \in W} c(\omega) \omega
$$


para cierto $c(\omega)$ de $\ell^{2}$. Para todo $v \in V$, se tiene que $\langle f, v\rangle=0$, por lo que

$$
\left\langle f_{1}, v\right\rangle=-\left\langle f_{2}, v\right\rangle \text {. }
$$

De esta igualdad y la hipótesis de ser marco se tiene que

$$
\left\|f_{1}\right\|^{2} \leq \frac{1}{A} \sum_{v \in V}\left|\left\langle f_{1}, v\right\rangle\right|^{2}=\frac{1}{A} \sum_{v \in V}=\frac{1}{A} \sum_{u \in U}\left|\left\langle f_{2}, u\right\rangle\right|^{2}=\frac{1}{A}\left\|f_{2}\right\|^{2} .
$$

Se obtiene así la desigualdad superior:

$$
\sum_{\omega \in W}|c(\omega)|^{2}=\|f\|^{2}=\left\|f_{1}\right\|^{2}+\left\|f_{2}\right\|^{2} \leq\left(1+\frac{1}{A}\right)\left\|f_{2}\right\|^{2} .
$$

La desigualdad inferior y la recíproca se prueban análogamente.

Extendemos el resultado de Olevskii y Ulanovskii a marcos de Parseval. En la prueba, la utilidad del gap se materializa a través de una proposición anterior, lo que aporta información sobre la cota inferior óptima.

Teorema 2.3.2. Sea $\left\{f_{i}\right\}_{i \in I}$ un marco de Parseval de $\mathcal{H}, \mathcal{M}$ un subespacio cerrado de $\mathcal{H}$ y $\sigma \subset$ I. Son equivalentes:

i. $\left\{P_{\mathcal{M}}\left(f_{i}\right)\right\}_{i \in \sigma}$ es una sucesión de Riesz.

ii. $\left\{P_{\mathcal{M}}^{\perp}\left(f_{i}\right)\right\}_{i \in \sigma^{c}}$ es una sucesión marco.

En particular, las cotas inferiores óptimas de ambas sucesiones coinciden.

Demostración. Sea $\left\{b_{i}\right\}_{i \in I}$ la base canónica ortonormal en $\ell^{2}(I)$. Sea $U: \ell^{2}(I) \rightarrow \mathcal{H}$ el operador de síntesis de $\left\{f_{i}\right\}_{i \in I}$ y sea $P_{\sigma}$ la proyección ortonormal (diagonal) sobre el subespacio generado por $\left\{b_{i}\right\}_{i \in \sigma}$. Notar que $U$ es una coisometría, esto es, $U U^{*}=I$.

Supongamos que $\mathcal{R}=\left\{P_{\mathcal{M}}\left(f_{i}\right)\right\}_{i \in \sigma}$ es una sucesión de Riesz con cota inferior $a>0$. Sea $W$ un isomorfismo isométrico

$$
W: \ell^{2}(\sigma) \rightarrow \overline{\operatorname{span}}\left\{b_{i}\right\}_{i \in \sigma} \subset \ell^{2}(I) .
$$

Entonces, el operador de síntesis $T_{\mathcal{R}}$ para $\left\{P_{\mathcal{M}}\left(f_{i}\right)\right\}_{i \in \sigma}$ está dado por $T_{\mathcal{R}}=P_{\mathcal{M}} U W$. Es claro que $P_{\mathcal{M}} U W=P_{\mathcal{M}} U P_{\sigma} W$. Luego, por la Proposición 2.1.7, tenemos que

$$
T_{\mathcal{R}}^{*} T_{\mathcal{R}}=W^{*} P_{\sigma} U^{*} P_{\mathcal{M}} U P_{\sigma} W \geq a I d_{\ell^{2}(\sigma)}=a W^{*} W
$$

Dado que $W$ es una isometría,

$$
P_{\sigma} U^{*} P_{\mathcal{M}} U P_{\sigma} \geq a P_{\sigma}
$$


Tomando $Q=U^{*} P_{\mathcal{M}} U$ y $P=P_{\sigma}$, por la Proposición 2.2.3 tenemos que

$$
Q^{\perp} P^{\perp} Q^{\perp} \geq a Q^{\perp}
$$

que es lo mismo que decir que

$$
U^{*} P_{\mathcal{M}^{\perp}} U P_{\sigma^{c}} U^{*} P_{\mathcal{M}^{\perp}} U \geq a U^{*} P_{\mathcal{M}^{\perp}} U
$$

Esto implica que $P_{\mathcal{M}^{\perp}} U P_{\sigma^{c}} U^{*} P_{\mathcal{M}^{\perp}} \geq a P_{\mathcal{M}^{\perp}}$. Entonces, usando nuevamente la Proposición 2.1.7, tenemos que $\left\{P_{\mathcal{M}}^{\perp}\left(f_{i}\right)\right\}_{i \in \sigma^{c}}$ es una sucesión marco, con cota inferior de marco $a>0$. La prueba de la recíproca se puede hacer de la misma forma sin mayores dificultades.

Cabe destacar que en la demostración de la prueba de Olevskii y Ulanovskii ellos mostraron que si la cota inferior de la sucesión marco $\left\{P_{\mathcal{M}} f_{i}\right\}_{i \in \sigma}$ es a, entonces $\left\{P_{\mathcal{M}^{\perp}} f_{i}\right\}_{i \in \sigma^{c}}$ es una sucesión de Riesz con cota inferior $\frac{a}{a+1}$. Por nuestra parte, pudimos establecer que $a$ es cota inferior de ambas sucesiones. Notemos además que $a>\frac{a}{a+1}$. Una forma de dimensionar la precisión sobre la cota inferior queda ilustrado con la siguiente observación.

Observación 2.3.3. Notar que, dado que una base de Riesz es de Parseval si y sólo si es una base ortonormal, podemos interpretar que la magnitud $\varepsilon>0$ de un marco $\varepsilon$-casi Parseval mide 'cuánto difiere un marco de ser una base ortonormal'. El Teorema 2.3.2 implica que si $\mathcal{H}=\mathcal{H}_{1} \oplus \mathcal{H}_{2}$ y $\mathcal{F}=\left\{f_{i}\right\}_{i \in I}$ es un marco de Parseval, para cierto $\varepsilon>0$, son equivalentes:

- $\left\{P_{\mathcal{H}_{1}}\left(f_{i}\right)\right\}_{i \in \sigma}$ es $\varepsilon$-casi Parseval en su espacio generado.

- $\left\{P_{\mathcal{H}_{2}}^{\perp}\left(f_{i}\right)\right\}_{i \in \sigma^{c}}$ es $\varepsilon$-casi base ortonormal en su espacio generado.

Así, si $\left\{P_{\mathcal{H}_{1}}\left(f_{i}\right)\right\}_{i \in \sigma}$ es $\varepsilon$-casi Parseval con $\varepsilon<\frac{1}{10}$, su cota inferior resulta $a=$ $1-\varepsilon>0,9$. Con la cota inferior que deducimos de la prueba de Olevskii-Ulanosvkii se pierde información respecto a la cota inferior de la sucesión $\left\{P_{\mathcal{H}_{2}}^{\perp}\left(f_{i}\right)\right\}_{i \in \sigma^{c}}$, sólo podríamos afirmar que la cota inferior es mayor a $\frac{1}{1+\frac{9}{10}}=\frac{9}{19} \simeq 0,47$.

\subsubsection{Condición del espectro del Grammiano}

En [20], los autores dieron respuesta a ciertos problemas relacionados a la descomposición de marcos en conjuntos linealmentes independientes. En uno de sus resultados preliminares notaron que, dado un marco de Parseval, las subfamilias de ese marco que satisfacen una condición de densidad se pueden caracterizar a partir de un determinado autovalor de las compresiones del grammiano.

Proposición 2.3.4. [20] Si $\mathcal{F}=\left\{f_{i}\right\}_{i \in I}$ un marco de Parseval para $\mathcal{H}, G_{\mathcal{F}}=T_{\mathcal{F}}^{*} T_{\mathcal{F}}$ su grammiano y $\sigma \subset I$, entonces

$\overline{\operatorname{span}}\left\{f_{i}\right\}_{i \in \sigma}=\mathcal{H}$ si sólo si 1 no es autovalor de $P_{\sigma^{c}} G_{\mathcal{F}} P_{\sigma^{c}}$. 
Inspirados en el antedicho resultado podemos dar una equivalencia de marco sobre las subfamilias de un marco de Parseval considerando el espectro del operador $P_{\sigma^{c}} G_{\mathcal{F}} P_{\sigma^{c}}$. La prueba es inmediata aplicando el Teorema 2.2.4 de la sección anterior.

Proposición 2.3.5. Sea $\mathcal{F}=\left\{f_{i}\right\}_{i \in I}$ un marco de Parseval para $\mathcal{H}, G_{\mathcal{F}}$ su grammiano y $\sigma \subset I$, entonces

$$
\left\{f_{i}\right\}_{i \in \sigma} \text { es marco si sólo si } 1 \text { no pertenece al espectro de } P_{\sigma^{c}} G P_{\sigma^{c}} \text {. }
$$

Demostración. Por Teorema 2.2.4,

$$
\left\{f_{i}\right\}_{i \in \sigma} \text { es marco en } \mathcal{H} \text { es equivalente a que } \delta\left(R\left(T_{\mathcal{F}}^{*}\right), R\left(P_{\sigma}\right)\right)<1
$$

pero

$$
\delta\left(R\left(T_{\mathcal{F}}^{*}\right), R\left(P_{\sigma}\right)\right)=\left\|P_{\sigma^{c}} G_{\mathcal{F}}\right\|^{2}=\left\|P_{\sigma^{c}} G_{\mathcal{F}} P_{\sigma^{c}}\right\|
$$

de donde se sigue el resultado enunciado.

En el siguiente ejemplo se considera un marco de parseval $\mathcal{F}=\left\{f_{i}\right\}_{i \in \mathbb{N}}$ tal que para cierto conjunto $\sigma \subset \mathbb{N}$, el subespacio generado por $\left\{f_{i}\right\}_{i \in \sigma}$ es un denso que no es un marco. Además, se ve que 1 , que no es un autovalor de $P_{\sigma^{c}} G_{\mathcal{F}} P_{\sigma^{c}}$, pertenece al espectro.

Ejemplo 2.3.6. Sea $\mathcal{H}$ un espacio de Hilbert y $\left\{e_{i}\right\}_{i \in \mathbb{N}}$ una base ortonormal. Sea $\left\{\theta_{n}\right\}_{n \in \mathbb{N}}$ una sucesión de números reales tal que $\operatorname{lím}_{n \rightarrow+\infty} \theta_{n}=\frac{\pi}{2}$. Consideremos la sucesión $\mathcal{F}=\left\{f_{n}\right\}_{n \in \mathbb{N}}$ dada por

$$
f_{2 k-1}=\cos \left(\theta_{k}\right) e_{k} \quad \mathrm{y} \quad f_{2 k}=\operatorname{sen}\left(\theta_{k}\right) e_{k}
$$

Es claro que $\mathcal{F}$ es un marco de Parseval ya que para todo $x \in \mathcal{H}$,

$$
\begin{aligned}
\sum_{n \in \mathbb{N}}\left|\left\langle x, f_{n}\right\rangle\right|^{2} & =\sum_{i \in \mathbb{N}} \cos ^{2}\left(\theta_{k}\right)\left|\left\langle x, e_{k}\right\rangle\right|^{2}+\operatorname{sen}^{2}\left(\theta_{k}\right)\left|\left\langle x, e_{k}\right\rangle\right|^{2} \\
& =\sum_{i \in \mathbb{N}}\left|\left\langle x, e_{k}\right\rangle\right|^{2}=\|x\|^{2} .
\end{aligned}
$$

Si consideramos $\sigma=\{2 k-1\}_{k \in \mathbb{N}}$, la sucesión

$$
\left\{f_{i}\right\}_{i \in \sigma}=\left\{\cos \left(\theta_{k}\right) e_{k}\right\}_{k \in \mathbb{N}}
$$

es claramente densa en $\mathcal{H}$. Sin embargo, no resulta un marco para $\mathcal{H}$ pues

$$
\sum_{i \in \sigma}\left|\left\langle e_{j}, f_{i}\right\rangle\right|^{2}=\cos ^{2}\left(\theta_{j}\right) \rightarrow 0
$$


Por otro lado,

$G_{\mathcal{F}}=\sum_{k \in \mathbb{N}} \cos ^{2}\left(\theta_{k}\right) e_{2 k-1} \otimes e_{2 k-1}+\cos \left(\theta_{k}\right) \operatorname{sen}\left(\theta_{k}\right)\left(e_{2 k} \otimes e_{2 k-1}+e_{2 k-1} \otimes e_{2 k}\right)+\operatorname{sen}^{2}\left(\theta_{2 k}\right) e_{2 k} \otimes e_{2 k}$.

Matricialmente, en la base $\left\{e_{j}\right\}_{j \in \mathbb{N}}$,

$G_{\mathcal{F}}=\left[\begin{array}{ccccc}\cos ^{2}\left(\theta_{1}\right) & \cos \left(\theta_{1}\right) \operatorname{sen}\left(\theta_{1}\right) & & & \\ \cos \left(\theta_{1}\right) \operatorname{sen}\left(\theta_{1}\right) & \operatorname{sen}^{2}\left(\theta_{1}\right) & \cos ^{2}\left(\theta_{2}\right) & \cos \left(\theta_{2}\right) \operatorname{sen}\left(\theta_{2}\right) & \\ & & \cos \left(\theta_{2}\right) \operatorname{sen}\left(\theta_{2}\right) & \operatorname{sen}^{2}\left(\theta_{2}\right) & \\ & & & \ddots & \\ & & & & \ddots\end{array}\right]$.

Por lo tanto,

$$
P_{\sigma^{c}} G_{\mathcal{F}} P_{\sigma^{c}}=\sum_{k \in \mathbb{N}} \operatorname{sen}^{2}\left(\theta_{k}\right) e_{2 k} \otimes e_{2 k}
$$

es un operador diagonal y su espectro ${\overline{\left\{\operatorname{sen}^{2}\left(\theta_{k}\right)\right.}}_{k \in \mathbb{N}}$ contiene a 1 . 


\section{Capítulo 3}

\section{Marcos Woven}

En este capítulo estudiamos una familia de marcos que representa otra forma de recurrir a la redundancia en el contexto de marcos, los llamados marcos woven. Como mencionamos anteriormente, la redundancia es una característica típica de los marcos que incentiva su presencia en las aplicaciones. En algunos problemas resulta de utilidad contar con más de un conjunto de vectores que sea marco, es decir, con más de un marco para un mismo espacio. Hacemos mención de un problema de aplicación para dar una idea inicial.

Supongamos que se tiene una placa de metal de la cual necesitamos medir la temperatura en cada punto de la superficie. Para eso, se instala un conjunto de sensores $\mathcal{F}=\left\{f_{i}\right\}_{i=1}^{N}$ que permite obtener dicha temperatura utilizando sus $N$ elementos. Para mayor precisión, se instala otro sistema de sensores $\mathcal{G}=\left\{g_{i}\right\}_{i=1}^{N}$ con las mismas características de $\mathcal{F}$ pero ubicado en otros puntos de la placa. Si tomamos cierto $\sigma \subset\{1, \ldots, N\}$ y consideramos los sensores $\sigma$ de $\mathcal{F}$ y los sensores $\sigma^{c}$ de $\mathcal{G}$, ¿cómo tienen que ser $\mathcal{F}$ y $\mathcal{G}$ para que podamos obtener la temperatura deseada con $\left\{f_{i}\right\}_{i \in \sigma} \cup\left\{g_{i}\right\}_{i \in \sigma^{c}}$ independientemente del $\sigma$ elegido?

En términos de marcos, se puede pensar este problema de la siguiente manera: sean $\mathcal{F}=\left\{f_{i}\right\}_{i \in I}$ y $\mathcal{G}=\left\{g_{i}\right\}_{i \in I}$ dos marcos para un espacio de Hilbert $\mathcal{H}$. Se buscan condiciones sobre $\mathcal{F}$ y $\mathcal{G}$ de manera que para todo $\sigma \subset I,\left\{f_{i}\right\}_{i \in \sigma} \cup\left\{g_{i}\right\}_{i \in \sigma^{c}}$ sea un marco para $\mathcal{H}$.

La noción de familias de marcos woven fue presentada por primera vez por Bemrose, Casazza, Gröchenig, Lammers y Lynch en 2015 [15]. Una de las dificultades de esta noción se presenta al momento de obtener cotas de marcos que no dependan de $\sigma$. En el caso más simple, se cuenta con pares de marcos $\{\mathcal{F}, \mathcal{G}\}$ y se desea obtener cotas uniformes para los marcos $\left\{f_{i}\right\}_{i \in \sigma} \cup\left\{g_{i}\right\}_{i \in \sigma^{c}}$. Es por esto que los autores consideraron apropiado definir el término débilmente woven si las cotas de marco dependen de cuáles elementos de cada marco se consideran. Los autores probaron que las nociones de woven y débilmente woven son equivalentes para pares de marcos, para familias de marcos $\left\{\mathcal{F}_{i}\right\}_{i=1}^{N}$ con $N>2$ resulta un problema abierto. Actualmente, el estudio de marcos woven se encuentra en aumento, como se puede apreciar en los numerosos trabajos que surgieron en relación a nociones de marcos, como marcos woven en es- 
pacios de Banach [28], así como generalizaciones, marcos continuos en [100] o marcos woven de fusión [45], entre otros.

Exhibimos en este capítulo resultados para pares de marcos woven, entre los que destacamos un resultado que mejora una condición de suficiencia de [15] y una primera condición necesaria en esta noción inspirada en las propiedades de ángulos de subespacios de los marcos de Riesz.

\subsection{Definición de woven y principales resultados}

Los marcos woven resultan una noción relativamente reciente. Exhibiremos a continuación las definiciones generales y algunos de los principales resultados. Para $M \in \mathbb{N}$ denotaremos por $[M]$ al conjunto $\{1,2, \cdots, M\}$.

Definición 3.1.1. Sea $\mathcal{F}=\left\{F_{j}\right\}_{j=1}^{N}$ una familia de marcos $F_{j}=\left\{f_{i j}\right\}_{i \in I}$ y una partición $\left\{\sigma_{j}\right\}_{j \in[M]}$ de $I$.

- Se denomina weaving a la familia de vectores que se forma al considerar los elementos $\left\{f_{i j}\right\}_{i \in \sigma_{j}}$ de cada marco $F_{j}$.

- $\mathcal{F}$ es débilmente woven si para cada partición $\left\{\sigma_{j}\right\}_{j \in[M]}$ de I todo weaving $\left\{f_{i j}\right\}_{i \in \sigma_{j}}$ es un marco con cotas $A_{\sigma}, B_{\sigma}$.

Definición 3.1.2. Una familia de marcos $\left\{f_{i j}\right\}_{i \in I, j \in[M]}$ es woven si es débilmente woven y existen $A, B>0$ tal que para toda partición $\left\{\sigma_{j}\right\}_{j \in[M]}$ de $I$,

$$
A\|x\|^{2} \leq \sum_{j=1}^{M} \sum_{i \in \sigma_{j}}\left|\left\langle x, f_{i j}\right\rangle\right|^{2} \leq B\|x\|^{2} .
$$

Es decir, si existen cotas de marco uniformes $A, B$ para todo weaving.

Si contamos con familias finitas de marcos donde cada weaving es un marco, la uniformidad que demanda la definición de marcos woven respecto a la cota superior, se obtiene fácilmente.

Proposición 3.1.3. Sean $F_{j}=\left\{f_{i j}\right\}_{i \in I}$ sucesiones de Bessel para $\mathcal{H}$ con cotas $B_{j}$ para todo $j \in[M]$. Todo weaving de $\left\{F_{j}\right\}_{j=1}^{N}$ es una sucesión de Bessel con cota $\sum_{j=1}^{M} B_{j}$.

Demostración. Para cada partición $\left\{\sigma_{j}\right\}_{j \in[M]}$ de $I$ y todo $x \in \mathcal{H}$,

$$
\sum_{j=1}^{M} \sum_{i \in \sigma_{j}}\left|\left\langle x, f_{i j}\right\rangle\right|^{2} \leq \sum_{j=1}^{M} \sum_{i \in I}\left|\left\langle x, f_{i j}\right\rangle\right|^{2} \leq \sum_{j=1}^{M} B_{j} \cdot\|x\|^{2} .
$$


Es natural preguntarse cuándo las nociones de débilmente woven y woven son equivalentes. En dimensión finita la respuesta es trivial porque hay una cantidad finita de particiones de un conjunto de índices.

Teorema 3.1.4. Una familia de marcos $\left\{f_{i j}\right\}_{i \in I, j \in[M]}$ para un espacio de Hilbert $\mathcal{H}$ de dimensión finita es woven si y sólo si para toda partición $\left\{\sigma_{j}\right\}_{j \in[M]}$ de $I,\left\{f_{i j}\right\}_{i \in \sigma_{j}}$ es un marco para $\mathcal{H}$.

En dimensión infinita, las condiciones que se deben pedir sobre la familia de marcos para asegurar equivalencia entre woven y débilmente woven se encuentran aún en estudio. Los autores de [15] pudieron probar la equivalencia para pares de marcos woven sin considerar hipótesis extras. Las técnicas empleadas para la prueba consisten en el análisis de una serie de condiciones sobre el conjunto de índices. Dichas técnicas no parecen extenderse a familias con una mayor cantidad de marcos.

Teorema 3.1.5. Sean $\mathcal{F}=\left\{f_{i}\right\}_{i \in I}$ y $\mathcal{G}=\left\{g_{i}\right\}_{i \in I}$ marcos para un espacio de Hilbert $\mathcal{H}$. Son equivalentes:

- $\{\mathcal{F}, \mathcal{G}\}$ es débilmente woven.

- $\{\mathcal{F}, \mathcal{G}\}$ es woven.

\section{2. $\quad$ Pares woven y operadores}

En esta sección centramos nuestro estudio en familias de pares de marcos proponiendo un enfoque de operadores al problema. Retomamos la definición de woven, ahora para pares de marcos:

Definición 3.2.1. Sean $\mathcal{F}=\left\{f_{i}\right\}_{i \in I}$ y $\mathcal{G}=\left\{g_{i}\right\}_{i \in I}$ dos marcos para un espacio de Hilbert $\mathcal{H}$. Un par $(\mathcal{F}, \mathcal{G})$ es woven si existen $A, B>0$ tales que para todo $\sigma \subset I$,

$$
A\|x\|^{2} \leq \sum_{i \in \sigma}\left|\left\langle x, f_{i}\right\rangle\right|^{2}+\sum_{i \in \sigma^{c}}\left|\left\langle x, g_{i}\right\rangle\right|^{2} \leq B\|x\|^{2}
$$

para todo $x \in \mathcal{H}$.

Es fácil construir pares de marcos woven y no woven:

\section{Ejemplo 3.2.2.}

- Si $\mathcal{F}=\left\{f_{i}\right\}_{i \in I}$ es un marco para un Hilbert $\mathcal{H},(\mathcal{F}, \mathcal{F})$ es un par woven.

- En particular, si $\mathcal{E}=\left\{e_{i}\right\}_{i=1}^{\infty}$ es una base ortonormal, $(\mathcal{E}, \mathcal{E})$ es un par woven.

- Por otro lado, $\left\{e_{i}\right\}_{i=1}^{\infty}$ y $\left\{e_{2}, e_{1}\right\} \cup\left\{e_{i}\right\}_{i=3}^{\infty}$ no son woven. 
Podemos presentar la definición de pares woven en términos de los operadores de síntesis de cada marco. Esta traducción al lenguaje de operadores será de gran utilidad para nuestros resultados venideros. Para $\sigma \subset I$, denotaremos por $P_{\sigma}$ a la proyección ortogonal sobre $\overline{\operatorname{span}}_{n \in \sigma}\left\{e_{n}\right\}$.

Proposición 3.2.3. Sean $\mathcal{F}=\left\{f_{i}\right\}_{i \in I}$ y $\mathcal{G}=\left\{g_{i}\right\}_{i \in I}$ marcos para un espacio de Hilbert $\mathcal{H}$ con operadores de sintesis $T_{\mathcal{F}}$ y $T_{\mathcal{G}}$, respectivamente. $(\mathcal{F}, \mathcal{G})$ es un par woven si y sólo si $T_{\mathcal{G}} P_{\sigma}+T_{\mathcal{F}} P_{\sigma^{c}}$ es suryectivo para todo $\sigma \subset I$.

Demostración. Dado $\sigma \subset I$, consideremos $\left\{g_{i}\right\}_{i \in \sigma} \cup\left\{f_{i}\right\}_{i \in \sigma^{c}}$. Por la Proposición 1.3.3 tenemos que

$$
\begin{gathered}
\left\{g_{i}\right\}_{i \in \sigma} \cup\left\{f_{i}\right\}_{i \in \sigma^{c}} \text { es un marco } \\
\mathbb{1} \\
T_{\mathcal{G}} P_{\sigma}+T_{\mathcal{F}} P_{\sigma^{c}}=T_{\mathcal{G}} P_{\sigma}+T_{\mathcal{F}}\left(I-P_{\sigma}\right) \text { es suryectivo. }
\end{gathered}
$$

Con lo cual, por Teorema 3.1.5, $(\mathcal{F}, \mathcal{G})$ es woven si y sólo si $T_{\mathcal{G}} P_{\sigma}+T_{\mathcal{F}}\left(I-P_{\sigma}\right)$ es suryectivo para todo $\sigma \subset I$.

\subsubsection{Condiciones suficientes}

Las condiciones suficientes que exhiben los autores de [15] para que un par de marcos sea woven consisten en desigualdades relacionadas con las cotas óptimas de cada marco junto con un resultado de perturbación.

Teorema 3.2.4. Sean $\mathcal{F}=\left\{f_{i}\right\}_{i \in I}$ y $\mathcal{G}=\left\{g_{i}\right\}_{i \in I}$ marcos para un espacio de Hilbert $\mathcal{H}$ con cotas de marco $A_{\mathcal{F}}, B_{\mathcal{F}}$ y $A_{\mathcal{G}}, B_{\mathcal{G}}$, respectivamente. Sea $0<\lambda<1$ tal que

$$
\lambda\left(\sqrt{B_{\mathcal{F}}}+\sqrt{B_{\mathcal{G}}}\right) \leq \frac{A_{\mathcal{F}}}{2} .
$$

Supongamos que para toda sucesión de escalares $\left\{a_{i}\right\}_{i \in I}$ se tiene

$$
\left\|\sum_{i \in I} a_{i}\left(f_{i}-g_{i}\right)\right\| \leq \lambda\left\|\left\{a_{i}\right\}_{i \in I}\right\|_{2}
$$

entonces $(\mathcal{F}, \mathcal{G})$ es un par woven.

Proposición 3.2.5. (Cor 6.2 [15]) Sea $\mathcal{F}=\left\{f_{i}\right\}_{i \in I}$ un marco para un espacio de Hilbert $\mathcal{H}$ y sea $T \in L(\mathcal{H})$ tal que

$$
\|I d-T\|^{2}<\frac{A_{\mathcal{F}}}{B_{\mathcal{F}}}
$$

para $i \in I$, entonces $\left\{f_{i}\right\}_{i \in I}$ y $\left\{T f_{i}\right\}_{i \in I}$ son woven. 
Notar que de la desigualdad (3.4) se obtiene la inversibilidad de $T$. Una mejora de este resultado es consecuencia del Teorema 3.2.7.

El siguiente resultado que usaremos en esta sección es un caso particular de lo desarrollado por J. Ding y L. Huan en [46].

Lema 3.2.6. [37] Sea $A \in L(\mathcal{H})$ un operador suryectivo, con inversa de MoorePenrose $A^{\dagger}$. Si $B \in L(\mathcal{H})$ es tal que $\|A-B\|<\left\|A^{\dagger}\right\|^{-1}=\gamma(A)$, entonces $B$ es un operador suryectivo con $\gamma(B) \geq \gamma(A)-\|A-B\|$.

Demostración. Debido a la suryectividad de $A$ se tiene que $A A^{\dagger}=I$. Como

$$
\left\|B A^{\dagger}-I\right\|=\left\|(B-A) A^{\dagger}\right\| \leq\|B-A\|\left\|A^{\dagger}\right\|<1,
$$

$B A^{\dagger}$ es inversible. Más aún, por el clásico resultado de Neumann,

$$
\left(B A^{\dagger}\right)^{-1}=\sum_{n=0}^{\infty}\left(I-B A^{\dagger}\right)^{n}
$$

Por lo que,

$$
\left\|\left(B A^{\dagger}\right)^{-1}\right\| \leq \sum_{n=0}^{\infty}\left\|\left(I-B A^{\dagger}\right)^{n}\right\| \leq \sum_{n=0}^{\infty}\|A-B\|^{n}\left\|A^{\dagger}\right\|^{n} .
$$

Como el operador $A^{\dagger}\left(B A^{\dagger}\right)^{-1}$ es una inversa a derecha de $B, B$ resulta un operador suryectivo. Dado que la inversa de Moore-Penrose es la que tiene la norma mínima sobre las pseudoinversas, $\left\|B^{\dagger}\right\| \leq\left\|A^{\dagger}\left(B A^{\dagger}\right)^{-1}\right\|$. Luego,

$$
\begin{aligned}
\gamma(B)=\left\|B^{\dagger}\right\|^{-1} & \geq\left\|A^{\dagger}\left(B A^{\dagger}\right)^{-1}\right\|^{-1} \geq \gamma(A)\left\|\left(B A^{\dagger}\right)^{-1}\right\|^{-1} \\
& \stackrel{(3.5)}{\geq} \gamma(A)\left(\sum_{n=0}^{\infty}\|A-B\|^{n}\left\|A^{\dagger}\right\|^{n}\right)^{-1} \\
& =\gamma(A)\left(1-\|A-B\|\left\|A^{\dagger}\right\|\right)=\gamma(A)-\|A-B\|>0 .
\end{aligned}
$$

Teorema 3.2.7. Sean $\mathcal{F}=\left\{f_{i}\right\}_{i \in I}$ y $\mathcal{G}=\left\{g_{i}\right\}_{i \in I}$ marcos para un espacio de Hilbert $\mathcal{H}$ con operadores de sintesis $T_{\mathcal{F}}$ y $T_{\mathcal{G}}$, respectivamente. Si $A_{\mathcal{F}}$ es la cota inferior de marco de $\mathcal{F}$ y $\left\|T_{\mathcal{F}}-T_{\mathcal{G}}\right\|<\sqrt{A_{\mathcal{F}}}$, entonces $(\mathcal{F}, \mathcal{G})$ es un par woven con cotas de $\operatorname{marco}\left(A_{\mathcal{F}}^{1 / 2}-\left\|T_{\mathcal{F}}-T_{\mathcal{G}}\right\|\right)^{2}$ y $B_{\mathcal{F}}+B_{\mathcal{G}}$.

Demostración. Dado $\sigma \subset I$, sea $\mathcal{W}_{\sigma}$ un weaving de $\mathcal{F}$ y $\mathcal{G}$. Por la Proposición 3.1.3, tenemos que la cota superior de $\mathcal{W}_{\sigma}$ es $B_{\mathcal{F}}+B_{\mathcal{G}}$.

Es claro de (3.1) que, para cada $\sigma \subset I$, el operador de síntesis del weaving correspondiente es

$$
T_{\mathcal{W}_{\sigma}}:=T_{\mathcal{F}}\left(I d-P_{\sigma}\right)+T_{\mathcal{G}} P_{\sigma}=T_{\mathcal{F}}+\left(T_{\mathcal{G}}-T_{\mathcal{F}}\right) P_{\sigma}
$$


Ahora bien, como

$$
\left\|T_{\mathcal{F}}-T_{\mathcal{W}_{\sigma}}\right\|=\left\|\left(T_{\mathcal{F}}-T_{\mathcal{G}}\right) P_{\sigma}\right\| \leq\left\|\left(T_{\mathcal{F}}-T_{\mathcal{G}}\right)\right\|<A_{\mathcal{F}}^{1 / 2}=\left\|T_{\mathcal{F}}^{\dagger}\right\|^{-1}
$$

por Lema 3.2.6, tenemos que $T_{\mathcal{W}_{\sigma}}$ es un operador suryectivo. Más aún,

$$
\left\|T_{\mathcal{W}_{\sigma}}^{\dagger}\right\|<\left(A_{\mathcal{F}}^{1 / 2}-\left\|\left(T_{\mathcal{F}}-T_{\mathcal{G}}\right) P_{\sigma}\right\|\right)^{-1}
$$

Por lo tanto, por Proposición 1.3.2, el weaving $\mathcal{W}_{\sigma}$ es un marco para $\mathcal{H}$ con cota inferior $A_{\mathcal{W}_{\sigma}} \geq\left(A_{\mathcal{F}}^{1 / 2}-\left\|\left(T_{\mathcal{F}}-T_{\mathcal{G}}\right) P_{\sigma}\right\|\right)^{2}$. Dado que para todo $\sigma$,

$$
A_{\mathcal{F}}^{1 / 2}-\left\|T_{\mathcal{F}}-T_{\mathcal{G}}\right\| \leq A_{\mathcal{F}}^{1 / 2}-\left\|\left(T_{\mathcal{F}}-T_{\mathcal{G}}\right) P_{\sigma}\right\|
$$

resulta que $A=\left(A_{\mathcal{F}}^{1 / 2}-\| T_{\mathcal{F}}-T_{\mathcal{G}}\right)$

A partir de este resultado, se derivan fácilmente afirmaciones sobre perturbaciones del tipo Paley-Wiener (ver [36]).

Corolario 3.2.8. Sean $\mathcal{F}=\left\{f_{i}\right\}_{i \in I}$ y $\mathcal{G}=\left\{g_{i}\right\}_{i \in I}$ marcos para $\mathcal{H}$ con cotas de marco $A_{\mathcal{F}}, B_{\mathcal{F}}$ y $A_{\mathcal{G}}, B_{\mathcal{G}}$, respectivamente. Sean $\lambda, \mu, \eta>0$ tales que

$$
\lambda \sqrt{B_{\mathcal{F}}}+\mu \sqrt{B_{\mathcal{G}}}+\eta<\sqrt{A_{\mathcal{F}}}
$$

y para toda sucesión $\left\{a_{i}\right\}_{i \in I}$ de escalares

$$
\left\|\sum_{i \in I} a_{i}\left(f_{i}-g_{i}\right)\right\| \leq \lambda\left\|\sum_{i \in I} a_{i} f_{i}\right\|+\mu\left\|\sum_{i \in I} a_{i} g_{i}\right\|+\eta\left\|\sum_{i \in I}\left|a_{i}\right|^{2}\right\|^{1 / 2},
$$

entonces el par $(\mathcal{F}, \mathcal{G})$ es woven.

Demostración. Tomando supremos sobre sucesiones $\left\{a_{i}\right\}_{i \in I}$ de norma 1 en (3.6) tenemos que

$$
\left\|T_{\mathcal{F}}-T_{\mathcal{G}}\right\| \leq \lambda \sqrt{B_{\mathcal{F}}}+\mu \sqrt{B_{\mathcal{G}}}+\eta<\sqrt{A_{\mathcal{F}}},
$$

por lo que la conclusión se sigue del resultado anterior.

Observación 3.2.9. Notar que nuestro Teorema 3.2.7 es más débil que el Teorema 3.2.4: si consideremos (3.2) y (3.3) en términos de los operadores de síntesis tenemos que

$$
\lambda\left(\left\|T_{\mathcal{F}}\right\|+\left\|T_{\mathcal{G}}\right\|\right)<\frac{A_{\mathcal{F}}}{2} \text { y }\left\|T_{\mathcal{F}}-T_{\mathcal{G}}\right\|<\lambda .
$$

Usando ambas hipótesis,

$$
\begin{aligned}
\left\|T_{\mathcal{F}}-T_{\mathcal{G}}\right\|^{2} & =\left\|T_{\mathcal{F}}-T_{\mathcal{G}}\right\|\left\|T_{\mathcal{F}}-T_{\mathcal{G}}\right\| \\
& \leq\left\|T_{\mathcal{F}}-T_{\mathcal{G}}\right\|\left(\left\|T_{\mathcal{F}}\right\|+\left\|T_{\mathcal{G}}\right\|\right) \\
& <\lambda\left(\left\|T_{\mathcal{F}}\right\|+\left\|T_{\mathcal{G}}\right\|\right)<\frac{A_{\mathcal{F}}}{2} .
\end{aligned}
$$


Luego $\left\|T_{\mathcal{F}}-T_{\mathcal{G}}\right\|<\sqrt{\frac{A_{\mathcal{F}}}{2}}<\sqrt{A_{\mathcal{F}}}$.

Al siguiente corolario lo consideramos una leve generalización de la Proposición 3.2 .5 .

Corolario 3.2.10. Sea $\mathcal{F}=\left\{f_{i}\right\}_{i \in I}$ un marco para un espacio de Hilbert $\mathcal{H}$ y sea $T \in L(\mathcal{H})$ tal que $\left\|(I d-T) T_{\mathcal{F}}\right\|<\sqrt{A_{\mathcal{F}}}$ para $i \in I$, entonces $\left(\left\{f_{i}\right\}_{i \in I},\left\{T f_{i}\right\}_{i \in I}\right)$ es un par woven.

Demostración. Se deduce del teorema simplemente notando que $T T_{\mathcal{F}}$ es el operador de síntesis de la sucesión de Bessel $\left\{T f_{i}\right\}_{i \in I}$.

La diferencia de este Corolario con la Proposición 3.2.5 se puede apreciar en el siguiente ejemplo.

Ejemplo 3.2.11. Sea $\left\{e_{i}\right\}_{i \in I}$ una base ortonormal de $\mathcal{H}$. Para $M>1$ consideremos el marco para $\mathcal{H}$ :

$$
\mathcal{F}=\left\{\sqrt{M} e_{1}, e_{2}, e_{3}, \ldots\right\}
$$

que tiene cotas de marco $A=1, B=M$. Notar que, según Proposición 3.2.5, para aquellos operadores inversibles $T$ tales que $\|I d-T\|^{2}<\frac{A}{B}=\frac{1}{M}$, se tiene que $(\mathcal{F}, T(\mathcal{F}))$ es woven. En particular, a medida que tomamos valores mayores para la cota superior $M, T$ se encuentra más próximo a $I d$.

Ahora, sea $0<c<1$ y

$$
T=e_{1} \otimes e_{1}+\sum_{i>1}(1-c) e_{i} \otimes e_{i}
$$

Claramente, $T$ es un inversible de $L(\mathcal{H})$ y $\left\|(I d-T) T_{\mathcal{F}}\right\|=c<1$. Luego, por Corolario 3.2.10, $(\mathcal{F}, T(\mathcal{F}))$ es woven. Notar que, en este caso, $\left\|(I d-T) T_{\mathcal{F}}\right\|=c$ está arbitrariamente cerca a 1 , independientemente del valor de $M$.

Concluimos esta sección con un resultado de "doble perturbación": dado un par de operadores inversibles, obtenemos una condición de suficiencia para que un par woven perturbado sea woven.

Teorema 3.2.12. Sean $\mathcal{F}=\left\{f_{i}\right\}_{i \in I}$ y $\mathcal{G}=\left\{g_{i}\right\}_{i \in I}$ marcos para $\mathcal{H}$ con cotas de marco $A_{\mathcal{F}}, B_{\mathcal{F}}$ y $A_{\mathcal{G}}, B_{\mathcal{G}}$ respectivamente tales que $(\mathcal{F}, \mathcal{G})$ es un par woven con cota óptima inferior $A_{\mathcal{F G}}>0$. Si $U, V \in L(\mathcal{H})$ son operadores inversibles tales que

$$
\left\|U^{-1} V-I d\right\|^{2}<\frac{A_{\mathcal{F G}}}{B_{\mathcal{G}}} \quad o \quad\left\|V^{-1} U-I d\right\|^{2}<\frac{A_{\mathcal{F G}}}{B_{\mathcal{F}}}
$$

entonces $(U(\mathcal{F}), V(\mathcal{G}))$ son woven.

Demostración. Supongamos que $\left\|U^{-1} V-I d\right\|^{2}<\frac{A_{\mathcal{F G}}}{B_{\mathcal{G}}}$, el otro caso se puede probar de formar similar. Como hicimos anteriormente, dado $\sigma \subset I$, nuestro objetivo es 
probar la suryectividad del operador

$$
U T_{\mathcal{F}}+\left(V T_{\mathcal{G}}-U T_{\mathcal{F}}\right) P_{\sigma},
$$

que corresponde con el operador de síntesis de la sucesión (de Bessel) $\left\{U f_{i}\right\}_{i \in \sigma^{c}} \cup$ $\left\{V g_{i}\right\}_{i \in \sigma}$.

Consideremos el weaving $\mathcal{W}_{\sigma}:=\left\{f_{i}\right\}_{i \in \sigma^{c}} \cup\left\{g_{i}\right\}_{i \in \sigma}$. Entonces, dado que $(\mathcal{F}, \mathcal{G})$ son pares woven tenemos que

$$
A_{\mathcal{F G}} \leq A_{\sigma}=\left\|T_{\mathcal{W}_{\sigma}}^{\dagger}\right\|^{-2}
$$

Como asumimos $\left\|U^{-1} V-I d\right\|^{2}<\frac{A_{\mathcal{F} \mathcal{G}}}{B_{\mathcal{G}}}$, tenemos que

$$
\begin{aligned}
\left\|U^{-1} V T_{\mathcal{G}}-T_{\mathcal{G}}\right\| & \leq\left\|U^{-1} V-I d\right\|\left\|T_{\mathcal{G}}\right\| \\
& \leq\left\|U^{-1} V-I d\right\| B_{\mathcal{G}}^{1 / 2}<A_{\mathcal{F} \mathcal{G}}^{1 / 2} \leq\left\|T_{\mathcal{W}_{\sigma}}^{\dagger}\right\|^{-1} .
\end{aligned}
$$

Con lo cual,

$$
\left\|T_{\mathcal{W}_{\sigma}}-\left(T_{\mathcal{F}}+\left(U^{-1} V T_{\mathcal{G}}-T_{\mathcal{F}}\right) P_{\sigma}\right)\right\|=\left\|\left(U^{-1} V T_{\mathcal{G}}-T_{\mathcal{G}}\right) P_{\sigma}\right\|<\left\|T_{\mathcal{W}_{\sigma}}^{\dagger}\right\|^{-1}
$$

Luego, por Lema 3.2.6, el operador $T_{\mathcal{F}}+\left(U^{-1} V T_{\mathcal{G}}-T_{\mathcal{F}}\right) P_{\sigma}$ es suryectivo, al igual que $U\left(T_{\mathcal{F}}+\left(U^{-1} V T_{\mathcal{G}}-T_{\mathcal{F}}\right) P_{\sigma}\right)$. Entonces el weaving $\left\{U f_{i}\right\}_{i \in \sigma^{c}} \cup\left\{V g_{i}\right\}_{i \in \sigma}$ resulta un marco $\mathcal{H}$ y por la equivalencia entre woven y débilmente woven, el par $(U(\mathcal{F}), V(\mathcal{G}))$ resulta woven.

Como consecuencia de este resultado obtenemos un efecto sobre los duales canónicos.

Corolario 3.2.13. Sean $\mathcal{F}=\left\{f_{i}\right\}_{i \in I}$ y $\mathcal{G}=\left\{g_{i}\right\}_{i \in I}$ marcos para $\mathcal{H}$ con cotas de marco $A_{\mathcal{F}}, B_{\mathcal{F}}$ y $A_{\mathcal{G}}, B_{\mathcal{G}}$ respectivamente tales que $(\mathcal{F}, \mathcal{G})$ es un par woven, con cota inferior $A_{\mathcal{F}, \mathcal{G}}>0$. Sean $S_{\mathcal{F}}$ y $S_{\mathcal{G}}$ los operadores de marco de $\mathcal{F}$ y $\mathcal{G}$, respectivamente. Entonces si

$$
\left\|S_{\mathcal{F}}^{-1}-S_{\mathcal{G}}^{-1}\right\|^{2}<A_{\mathcal{F}, \mathcal{G}} \operatorname{máx}\left\{\frac{1}{B_{\mathcal{G}} B_{\mathcal{F}}^{2}}, \frac{1}{B_{\mathcal{F}} B_{\mathcal{G}}^{2}}\right\}
$$

los duales canónicos $\left(\left\{S_{\mathcal{F}}^{-1} f_{i}\right\}_{i \in I},\left\{S_{\mathcal{G}}^{-1} g_{i}\right\}_{i \in I}\right.$ son un par woven.

En [45], Deepshikha, S. Garg, L. Vashish y G. Verma enuncian para marcos de fusión una condición que resulta interesante ya que depende de los coeficientes de marco de los vectores. Para contextualizar con nuestros resultados, lo enunciamos para marcos de vectores:

Teorema 3.2.14. (Teo 2.7 [45]) Sean $\mathcal{F}=\left\{f_{i}\right\}_{i \in I}$ y $\mathcal{G}=\left\{g_{i}\right\}_{i \in I}$ marcos para $\mathcal{H}$ con cotas de marco $A_{\mathcal{F}}, B_{\mathcal{F}}$ y $A_{\mathcal{G}}, B_{\mathcal{G}}$, respectivamente. Sea $M>0$ tal que para todo $\sigma \subset I$

$$
\sum_{i \in \sigma}\left|\left\langle x, f_{i}\right\rangle-\left\langle x, g_{i}\right\rangle\right|^{2} \leq M \min \left\{\sum_{i \in \sigma}\left|\left\langle x, f_{i}\right\rangle\right|^{2}, \sum_{i \in \sigma}\left|\left\langle x, g_{i}\right\rangle\right|^{2}\right\}
$$


para todo $x \in \mathcal{H}$. Entonces el par $(\mathcal{F}, \mathcal{G})$ es woven con cotas $\frac{A_{\mathcal{F}}+A_{\mathcal{G}}}{2 M+3} y B_{\mathcal{F}}+B_{\mathcal{G}}$.

Notemos que este teorema no se desprende de nuestro estudio de pares woven. Sin embargo, este enfoque desde el punto de vista de los operadores de análisis nos permite deducir el siguiente:

Teorema 3.2.15. Sean $\mathcal{F}=\left\{f_{i}\right\}_{i \in I}$ y $\mathcal{G}=\left\{g_{i}\right\}_{i \in I}$ marcos para $\mathcal{H}$ con cotas de marco $A_{\mathcal{F}}, B_{\mathcal{F}}$ y $A_{\mathcal{G}}, B_{\mathcal{G}}$, respectivamente. Supongamos que existe $0<M<1 / 2$ tal que

$$
\sum_{i \in I}\left|\left\langle x, f_{i}\right\rangle-\left\langle x, g_{i}\right\rangle\right|^{2} \leq M \operatorname{mín}\left\{\sum_{i \in I}\left|\left\langle x, f_{i}\right\rangle\right|^{2}, \sum_{i \in I}\left|\left\langle x, g_{i}\right\rangle\right|^{2}\right\}
$$

para todo $x \in \mathcal{H}$. Entonces el par $(\mathcal{F}, \mathcal{G})$ es woven con cotas $\left(\frac{1}{2}-M\right) A_{\mathcal{F}}$ y $B_{\mathcal{F}}+B_{\mathcal{G}}$.

Demostración. Dado $\sigma \subset I$, en términos de los operadores de análisis $T_{\mathcal{F}}^{*}$ y $T_{\mathcal{G}}^{*}$, se trata de probar que existe $c>0$ tal que

$$
\sum_{i \in \sigma}\left|\left\langle x, f_{i}\right\rangle\right|^{2}+\sum_{i \in \sigma}\left|\left\langle x, g_{i}\right\rangle\right|^{2}=\left\|P_{\sigma^{c}} T_{\mathcal{F}}^{*} x-P_{\sigma} T_{\mathcal{G}}^{*} x\right\|^{2} \geq c\|x\|^{2} .
$$

Reescribiendo el lado izquierdo, para $x \in \mathcal{H}$,

$$
\left\|P_{\sigma^{c}} T_{\mathcal{F}}^{*} x-P_{\sigma} T_{\mathcal{G}}^{*} x\right\|^{2}=\left\|T_{\mathcal{F}}^{*} x+P_{\sigma}\left(T_{\mathcal{F}}^{*}-T_{\mathcal{G}}^{*}\right) x\right\|^{2}
$$

Entonces

$$
\begin{aligned}
\left\|T_{\mathcal{F}}^{*} x+P_{\sigma}\left(T_{\mathcal{F}}^{*}-T_{\mathcal{G}}^{*}\right) x\right\|^{2} & \geq \frac{1}{2}\left\|T_{\mathcal{F}}^{*} x\right\|^{2}-\left\|P_{\sigma}\left(T_{\mathcal{F}}^{*}-T_{\mathcal{G}}^{*}\right) x\right\|^{2} \\
& \geq \frac{1}{2}\left\|T_{\mathcal{F}}^{*} x\right\|^{2}-\left\|\left(T_{\mathcal{F}}^{*}-T_{\mathcal{G}}^{*}\right) x\right\|^{2} \\
& \geq \frac{1}{2}\left\|T_{\mathcal{F}}^{*} x\right\|^{2}-M\left\|T_{\mathcal{F}}^{*} x\right\|^{2}=\left(\frac{1}{2}-M\right)\left\|T_{\mathcal{F}}^{*} x\right\|^{2} \\
& >\left(\frac{1}{2}-M\right) A_{\mathcal{F}}\|x\|^{2} .
\end{aligned}
$$

Notar que, respecto al resultado de Deepshikha et al, nuestra condición es restrictiva en la cota de $M<1 / 2$. Por otra parte, la cota (3.7) permite asegurar que el par sea woven sin la necesidad de verificarlo para cada $\sigma \subset I$.

\subsubsection{Gap y pares woven}

En [32], Casazza y Lynch definen subespacios a partir de los weavings para caracterizar a los pares de marcos que sean woven. Observamos que esas condiciones se pueden expresar en términos del gap entre subespacios. 
Proposición 3.2.16. (Cor 21 [32]) Sean $\mathcal{F}=\left\{f_{i}\right\}_{i \in I}$ y $\mathcal{G}=\left\{g_{i}\right\}_{i \in I}$ marcos para un espacio de Hilbert $\mathcal{H}$, son equivalentes:

1. $(\mathcal{F}, \mathcal{G})$ es un par woven

2. Para cada $\sigma \subset I$, sean

$$
W_{\sigma}=\overline{\operatorname{span}}\left\{f_{i}\right\}_{i \in \sigma} \quad \mathrm{y} \quad W_{\sigma}^{c}=\overline{\operatorname{span}}\left\{g_{i}\right\}_{i \in \sigma^{c}}
$$

y sea $P$ la proyección ortogonal sobre $W_{\sigma}^{\perp}$. Entonces

$$
\left.P\right|_{W_{\sigma}^{c}} \text { es sobre en } W_{\sigma}^{\perp} \text { y }\left\{P f_{i}\right\}_{i \in \sigma^{c}} \text { es un marco para } W_{\sigma}^{\perp} \text {. }
$$

Considerando un resultado del capítulo anterior podemos enunciar una versión con otras condiciones. Para eso, notar que la primera condición de (3.8) se puede reescribir como

$$
R\left(P_{W_{\sigma}^{\perp}} P_{W_{\sigma}^{c}}\right)=W_{\sigma^{\perp}}=R\left(P_{W_{\sigma}^{\perp}}\right)
$$

Por Corolario 2.2.2, esto equivale a pedir que

$$
\delta\left(W_{\sigma}^{\perp}, W_{\sigma}^{c}\right)<1
$$

Por otro lado, la segunda condición de (3.8), en términos del operador de síntesis $T_{\mathcal{F}}$, se traduce en que $P_{W_{\sigma}^{\perp}} T_{\mathcal{F}} P_{W_{\sigma}^{c}}$ sea suryectivo sobre $W_{\sigma^{\perp}}$. Como $P_{W_{\sigma}^{\perp}} T_{\mathcal{F}}$ es el operador de síntesis del marco $\left\{P_{W_{\sigma}^{\perp}} f_{i}\right\}_{i \in I}$, por Teorema 2.2.4, esto es equivalente a que $\delta\left(R\left(T_{\mathcal{F}}^{*} P_{W_{\sigma}^{\perp}}, R\left(P_{\sigma}\right)\right)<1\right.$. Observar que $R\left(T_{\mathcal{F}}^{*} P_{W_{\sigma}^{\perp}}\right)=T_{\mathcal{F}}^{*}\left(W_{\sigma}^{\perp}\right)$.

Por lo tanto, tenemos esta condición equivalente:

Proposición 3.2.17. Sean $\mathcal{F}=\left\{f_{i}\right\}_{i \in I}$ y $\mathcal{G}=\left\{g_{i}\right\}_{i \in I}$ marcos para un espacio de Hilbert $\mathcal{H}$, son equivalentes:

1. $(\mathcal{F}, \mathcal{G})$ es un par woven.

2. $\delta\left(W_{\sigma}^{\perp}, W_{\sigma}^{c}\right)<1$ y $\delta\left(T_{\mathcal{F}}^{*}\left(W_{\sigma}^{\perp}\right), R\left(P_{\sigma}\right)\right)<1$.

\subsection{Caso particular: marco dual canónico escaleado}

En el trabajo [82], los autores probaron un resultado para pares woven de g-marcos (Cor 5.4 [82]). Expresamos dicho resultado en término de los marcos vectoriales de este trabajo:

Proposición 3.3.1. Sea $\mathcal{F}=\left\{f_{i}\right\}_{i \in I}$ un marco para $\mathcal{H}$ con cotas $A_{\mathcal{F}}, B_{\mathcal{F}}$. Si $\frac{B_{\mathcal{F}}}{A_{\mathcal{F}}}<2$, entonces $\mathcal{F}$ y el marco dual canónico escaleado $\mathcal{G}=\left\{\frac{2 A_{\mathcal{F}} B_{\mathcal{F}}}{A_{\mathcal{F}}+B_{\mathcal{F}}} S_{\mathcal{F}}^{-1} f_{i}\right\}_{i \in I}$ son un par woven. 
El propósito de esta sección es aplicar el Teorema 3.2.7 a este caso particular. Específicamente, veremos que para un radio mayor $\frac{B_{\mathcal{F}}}{A_{\mathcal{F}}}$ podemos determinar un intervalo de números positivos $\alpha$ que garantizan que el par $\left(\mathcal{F}, \alpha \cdot \mathcal{F}^{\sharp}\right)$ es woven (denotando por $\mathcal{F}^{\sharp}$ al marco dual canónico de $\mathcal{F}$ ).

Teorema 3.3.2. Sea $\mathcal{F}=\left\{f_{i}\right\}_{i \in I}$ un marco para $\mathcal{H}$ con cotas $A_{\mathcal{F}}, B_{\mathcal{F}}$. Si $\frac{B_{\mathcal{F}}}{A_{\mathcal{F}}}<4$, entonces para todo $\alpha>0$ tal que

$$
B_{\mathcal{F}}-\sqrt{A_{\mathcal{F}} B_{\mathcal{F}}}<\alpha<2 A_{\mathcal{F}}
$$

se tiene que $\left(\mathcal{F}, \alpha \cdot \mathcal{F}^{\sharp}\right)$ es un par woven.

Demostración. Para usar el Teorema 3.2.7, es suficiente con probar que nuestras hipótesis implican que

$$
\left\|T_{\mathcal{F}}-\alpha S_{\mathcal{F}}^{-1} T_{\mathcal{F}}\right\|^{2}<A_{\mathcal{F}}
$$

Si reescribimos el lado izquierdo de esta desigualdad,

$$
\left\|T_{\mathcal{F}}-\alpha S_{\mathcal{F}}^{-1} T_{\mathcal{F}}\right\|^{2}=\left\|\left(I-\alpha S_{\mathcal{F}}^{-1}\right) T_{\mathcal{F}} T_{\mathcal{F}}^{*}\left(I-\alpha S_{\mathcal{F}}^{-1}\right)\right\|=\left\|\left(S_{\mathcal{F}}-\alpha I\right) S_{\mathcal{F}}^{-1}\left(S_{\mathcal{F}}-\alpha I\right)\right\| .
$$

Usando cálculo funcional, obtenemos explicítamente que

$$
\left\|\left(S_{\mathcal{F}}-\alpha I\right) S_{\mathcal{F}}^{-1}\left(S_{\mathcal{F}}-\alpha I\right)\right\|=\operatorname{máx}_{x \in\left[A_{\mathcal{F}}, B_{\mathcal{F}}\right]} \frac{(x-\alpha)^{2}}{x} .
$$

En particular,

$$
\left\|T_{\mathcal{F}}-\alpha S_{\mathcal{F}}^{-1} T_{\mathcal{F}}\right\|^{2}=\operatorname{máx}\left\{\frac{\left(B_{\mathcal{F}}-\alpha\right)^{2}}{B_{\mathcal{F}}}, \frac{\left(A_{\mathcal{F}}-\alpha\right)^{2}}{A_{\mathcal{F}}}\right\} .
$$

Como teníamos que $B_{\mathcal{F}}<4 A_{\mathcal{F}}$, entonces $B_{\mathcal{F}}-\sqrt{A_{\mathcal{F}} B_{\mathcal{F}}}<\sqrt{A_{\mathcal{F}} B_{\mathcal{F}}}$. Sea $\alpha$ tal que

$$
B_{\mathcal{F}}-\sqrt{A_{\mathcal{F}} B_{\mathcal{F}}}<\alpha \leq \sqrt{A_{\mathcal{F}} B_{\mathcal{F}}}
$$

Es fácil comprobar que $\alpha \leq \sqrt{A_{\mathcal{F}} B_{\mathcal{F}}}$ implica que

$$
\left\|T_{\mathcal{F}}-\alpha S_{\mathcal{F}}^{-1} T_{\mathcal{F}}\right\|^{2}=\frac{\left(B_{\mathcal{F}}-\alpha\right)^{2}}{B_{\mathcal{F}}} .
$$

Por otro lado, de $B_{\mathcal{F}}-\sqrt{A_{\mathcal{F}} B_{\mathcal{F}}}<\alpha$, tenemos que

$$
\frac{\left(B_{\mathcal{F}}-\alpha\right)^{2}}{B_{\mathcal{F}}}<\frac{\left(B_{\mathcal{F}}-\left(B_{\mathcal{F}}-\sqrt{A_{\mathcal{F}} B_{\mathcal{F}}}\right)\right)^{2}}{B_{\mathcal{F}}}=A_{\mathcal{F}},
$$

por lo que el par es woven.

De forma similar, si $\sqrt{A_{\mathcal{F}} B_{\mathcal{F}}}<\alpha<2 A_{\mathcal{F}}$, se deduce que

$$
\left\|T_{\mathcal{F}}-\alpha S_{\mathcal{F}}^{-1} T_{\mathcal{F}}\right\|^{2}=\frac{\left(A_{\mathcal{F}}-\alpha\right)^{2}}{A_{\mathcal{F}}}<A_{\mathcal{F}},
$$


por lo que nuevamente el Teorema 3.2.7 nos permite arribar a la conclusión buscada.

Observación 3.3.3. El teorema anterior no contempla el valor de la escala $\alpha=$ $\frac{2 A_{\mathcal{F}} B_{\mathcal{F}}}{A_{\mathcal{F}}+B_{\mathcal{F}}}$ utilizada en el resultado de [82]. Es claro que, por la desigualdad aritméticogeométrica, ese valor de $\alpha$ es menor o igual que $\sqrt{A_{\mathcal{F}} B_{\mathcal{F}}}$. Sin embargo, la condición $\frac{B_{\mathcal{F}}}{A_{\mathcal{F}}}<4$ no asegura que $B_{\mathcal{F}}-\sqrt{A_{\mathcal{F}} B_{\mathcal{F}}}<\alpha$ en este caso. A continuación analizamos esto cuidadosamente.

Dado que $\alpha \leq \sqrt{A_{\mathcal{F}} B_{\mathcal{F}}}$, queremos obtener condiciones sobre el cociente $\frac{B_{\mathcal{F}}}{A_{\mathcal{F}}}$ de manera que

$$
B_{\mathcal{F}}-\sqrt{A_{\mathcal{F}} B_{\mathcal{F}}}<\alpha
$$

y así arribar a la conclusión del Teorema 3.3.2. Haciendo un cálculo sencillo, vemos que la condición

$$
B_{\mathcal{F}}-\frac{2 A_{\mathcal{F}} B_{\mathcal{F}}}{A_{\mathcal{F}}+B_{\mathcal{F}}} \leq \sqrt{A_{\mathcal{F}} B_{\mathcal{F}}}
$$

es equivalente a tener

$$
\frac{B_{\mathcal{F}}-A_{\mathcal{F}}}{B_{\mathcal{F}}+A_{\mathcal{F}}}<\sqrt{\frac{A_{\mathcal{F}}}{B_{\mathcal{F}}}}
$$

Más aún, si tomamos $r=\frac{B_{\mathcal{F}}}{A_{\mathcal{F}}}$, esta desigualdad podemos expresarla como

$$
\frac{(r-1)^{2}}{(r+1)^{2}}<\frac{1}{r}
$$

En particular, nos interesa encontrar $r \geq 1$ tal que $r^{3}-3 r^{2}-r-1<0$. Como el polinomio $f(r)=r^{3}-3 r^{2}-r-1$ tiene una única raíz real

$$
r_{0}=\left(\sqrt[3]{\frac{3+\sqrt{\frac{11}{3}}}{6}}+\sqrt[3]{\frac{3-\sqrt{\frac{11}{3}}}{6}}\right)^{3} \approx 3,383
$$

podemos tomar cotas mayores a $\frac{B_{\mathcal{F}}}{A_{\mathcal{F}}}$ (esto es $\left.\frac{B_{\mathcal{F}}}{A_{\mathcal{F}}}<3,383\right)$ para obtener la misma conclusión que [82].

\subsection{Condición necesaria de ángulos}

El propósito de esta sección es exhibir condiciones vinculadas al ángulo entre subespacios para que un par de marcos sea woven. Uno de los antecedentes de ángulos y marcos es el trabajo [3]. En dicho trabajo se caracterizaron los llamados marcos de Riesz por medio de cotas uniformes sobre ángulos entre ciertos subespacios. Motiva esta sección encontrar condiciones de ese tipo en el contexto de pares woven.

Definición 3.4.1. Un marco $\mathcal{F}=\left\{f_{i}\right\}_{i \in I}$ se dice marco de Riesz si existe $C>0$ tal que para todo $\sigma \subset I,\left\{f_{i}\right\}_{i \in \sigma}$ es marco para $\mathcal{H}_{\sigma}=\overline{\operatorname{span}}_{i \in \sigma}\left\{f_{i}\right\}$ con cota $A_{\sigma} \geq C$. 
Proposición 3.4.2. [3] Sea $\mathcal{F}=\left\{f_{n}\right\}_{n \in \mathbb{N}}$ un marco con operador de sintesis $T_{\mathcal{F}}$. Son equivalentes

1. $\mathcal{F}$ es un marco de Riesz.

2. $N\left(T_{\mathcal{F}}\right)$ es compatible con respecto a la base $\mathcal{B}=\left\{e_{i}\right\}_{i \in I}$, esto es,

$$
\sup _{\sigma \subset I} c\left(N\left(T_{\mathcal{F}}\right), R\left(P_{\sigma}\right)\right)<1
$$

Similar a la equivalencia de operadores que notamos en la sección anterior, respecto a la definición de par de marcos woven, se puede obtener una condición de acotación inferior tomando el operador adjunto.

Lema 3.4.3. Sean $\mathcal{F}=\left\{f_{i}\right\}_{i \in I}$ y $\mathcal{G}=\left\{g_{i}\right\}_{i \in I}$ marcos para un espacio de Hilbert $\mathcal{H}$. Sean $A:=\left(T_{\mathcal{G}}-T_{\mathcal{F}}\right)^{*}$ y $B:=T_{\mathcal{F}}^{*}$.

$$
\begin{gathered}
(\mathcal{F}, \mathcal{G}) \text { es woven } \\
\mathbb{\Downarrow} \\
P_{\sigma} A+B \text { es acotado inferiormente para todo } \sigma \subset I .
\end{gathered}
$$

Es decir, que para todo $\sigma \subset I$, existe $C_{\sigma}>0$ tal que

$$
C_{\sigma}\|x\| \leq\left\|\left(P_{\sigma} A+B\right) x\right\| \text { para todo } x \in \mathcal{H} .
$$

Para el siguiente resultado consideraremos el espacio $\tilde{\mathcal{H}}=\mathcal{H} \oplus \mathcal{H}$ dotado con el producto interno

$$
\langle x \oplus y, z \oplus w\rangle_{\tilde{\mathcal{H}}}=\langle x, z\rangle+\langle y, w\rangle .
$$

Para $\sigma \subset I$, definimos la proyección oblicua $Q_{\sigma}: \tilde{\mathcal{H}} \rightarrow \tilde{\mathcal{H}}$ dada por

$$
Q_{\sigma}=\left(\begin{array}{cc}
I & P_{\sigma} \\
0 & 0
\end{array}\right)
$$

Por último, definimos $T_{\mathcal{F}, \mathcal{G}}: \tilde{\mathcal{H}} \rightarrow \mathcal{H}$ por

$$
T_{\mathcal{F}, \mathcal{G}}(x \oplus y)=T_{\mathcal{F}}(x)+\left(T_{\mathcal{F}}-T_{\mathcal{G}}\right)(y) .
$$

Teorema 3.4.4. Sean $\mathcal{F}=\left\{f_{i}\right\}_{i \in I}$ y $\mathcal{G}=\left\{g_{i}\right\}_{i \in I}$ marcos para un espacio de Hilbert $\mathcal{H}$. Si $(\mathcal{F}, \mathcal{G})$ es un par woven entonces

$$
\sup _{\sigma \subset I} c\left(N\left(T_{\mathcal{F}, \mathcal{G}}\right), R\left(Q_{\sigma}^{*}\right)\right)<1
$$

Demostración. Sean $B=T_{\mathcal{F}}^{*}$ y $A=T_{\mathcal{G}}^{*}-T_{\mathcal{F}}^{*}$ como en el lema anterior. Como $(\mathcal{F}, \mathcal{G})$ es woven, de (3.10) tenemos que existe $C>0$ tal que $C<\gamma\left(B+P_{\sigma} A\right)$. 
Ahora bien, veamos que $T_{\mathcal{F}, \mathcal{G}}^{*}$ tiene rango cerrado:

si $\left(T_{\mathcal{F}, \mathcal{G}}^{*}\left(x_{n}\right)\right)_{n}$ es una sucesión convergente entonces $\left(B x_{n}\right)_{n}$ es una sucesión convergente. Más aún, como $B$ es de rango cerrado, $B x_{n} \rightarrow B x$ para cierto $x$. Como

$$
\gamma(B)\left\|x-x_{n}\right\| \leq\left\|B\left(x-x_{n}\right)\right\|
$$

deducimos que $x_{n} \rightarrow x$, por lo que $A x_{n} \rightarrow A x$. Concluimos entonces que $T_{\mathcal{F}, \mathcal{G}}^{*}\left(x_{n}\right) \rightarrow T_{\mathcal{F}, \mathcal{G}}^{*}(x)$.

Es claro que $N\left(T_{\mathcal{F}, \mathcal{G}}^{*}\right)=\{0\}$. Notemos además que $T_{\mathcal{F}, \mathcal{G}}^{*}$ está acotado inferiormente ya que para $x \in \mathcal{H}$ con $\|x\|=1$,

$$
\left\|T_{\mathcal{F}, \mathcal{G}}^{*} x\right\|=\sqrt{\|B x\|^{2}+\|A x\|^{2}} \geq\|B x\| \geq \gamma(B)=\gamma\left(T_{\mathcal{F}}^{*}\right)=A_{\mathcal{F}}^{1 / 2} .
$$

Por lo que, $\gamma\left(T_{\mathcal{F}, \mathcal{G}}^{*}\right) \geq A_{\mathcal{F}}^{1 / 2}$. Ahora bien, dado que

$$
\gamma\left(Q_{\sigma} T_{\mathcal{F}, \mathcal{G}}^{*}\right)=\gamma\left(\left(\left(B+P_{\sigma} A\right) 0\right)^{\top}\right)=\gamma\left(B+P_{\sigma} A\right),
$$

si $\alpha=c\left(N\left(Q_{\sigma}\right), R\left(T_{\mathcal{F}, \mathcal{G}}^{*}\right)\right.$, por Proposición 1.1.4:

$$
\gamma\left(Q_{\sigma}\right) \gamma\left(T_{\mathcal{F}, \mathcal{G}}^{*}\right)\left(1-\alpha^{1 / 2}\right)^{1 / 2} \leq \gamma\left(B+P_{\sigma} A\right) \leq\left\|Q_{\sigma}\right\|\left\|T_{\mathcal{F}, \mathcal{G}}^{*}\right\|\left(1-\alpha^{1 / 2}\right)^{1 / 2} .
$$

De estas desigualdades tenemos que, si $(\mathcal{F}, \mathcal{G})$ woven, la cota uniforme de $\gamma\left(B+P_{\sigma} A\right)$ resulta equivalente a tener

$$
\sup _{\sigma \subset I} c\left(N\left(Q_{\sigma}\right), R\left(T_{\mathcal{F}, \mathcal{G}}^{*}\right)=\sup _{\sigma \subset I} c\left(N\left(T_{\mathcal{F}, \mathcal{G}}\right), R\left(Q_{\sigma}^{*}\right)\right)<1 .\right.
$$

Observación 3.4.5. La condición (3.11), que se la puede considerar como una condición de compatibilidad 'oblicua' entre $N\left(T_{\mathcal{F}, \mathcal{G}}\right)$ y los rangos de $Q_{\sigma}^{*}$, no garantiza que $(\mathcal{F}, \mathcal{G})$ sea un par woven ya que sólo implica que los weavings $\left\{f_{i}\right\}_{i \in \sigma^{c}} \cup\left\{g_{i}\right\}_{i \in \sigma}$ son sucesiones marcos para $\mathcal{H}$ (con cota inferior uniforme para todo $\sigma \subset I$ ).

\subsection{Una condición de transitividad}

Es claro de su definición que la noción de ser woven es reflexiva y simétrica, esto es, para todo marco $\mathcal{F}=\left\{f_{i}\right\}_{i \in I},(\mathcal{F}, \mathcal{F})$ es un par woven y si $\mathcal{G}=\left\{g_{i}\right\}_{i \in I}$ es otro marco tal que $(\mathcal{F}, \mathcal{G})$ es woven, es trivial que $(\mathcal{G}, \mathcal{F})$ también sea woven. Cabe preguntarse entonces respecto a la transitividad: sean $\mathcal{F}=\left\{f_{i}\right\}_{i \in I}, \mathcal{G}=\left\{g_{i}\right\}_{i \in I}$ y $\mathcal{J}=\left\{j_{i}\right\}_{i \in I}$ marcos para un espacio de Hilbert $\mathcal{H}$ tales que $(\mathcal{F}, \mathcal{G})$ y $(\mathcal{G}, \mathcal{J})$ son pares woven. En general, no resulta cierto que $(\mathcal{F}, \mathcal{J})$ sea woven como se ve en el siguiente ejemplo: 
Ejemplo 3.5.1. [94] Sea $\mathcal{H}=\mathbb{R}^{2}$ con $\left\{e_{1}, e_{2}\right\}$ una base ortonormal. Sean

$$
\mathcal{F}=\left\{e_{1}, e_{1}, e_{2}\right\}, \mathcal{G}=\left\{e_{1}, e_{2}, e_{2}\right\} \quad \text { y } \mathcal{J}=\left\{e_{1}, e_{2}, e_{1}\right\}
$$

marcos para $\mathcal{H}$. Es claro que $(\mathcal{F}, \mathcal{G})$ y $(\mathcal{G}, \mathcal{J})$ son pares woven con cotas uniformes $A=1$ y $B=2$. Sin embargo, $(\mathcal{F}, \mathcal{J})$ no resulta woven.

Veremos condiciones que ajustan las cotas planteadas por los autores de [94]. Introducimos algo de notación: fijado $x \in \mathcal{H}$, para una sucesión $\mathcal{F}=\left\{f_{i}\right\}_{i \in I}$ de $\mathcal{H}$

- denotamos por $M_{\mathcal{F}}$ a la suma

$$
M_{\mathcal{F}}:=\sum_{i \in I}\left|\left\langle x, f_{i}\right\rangle\right|^{2}
$$

- dado $\sigma \subset I$, notaremos por $M_{\mathcal{F}}^{\sigma}$ a $M_{\mathcal{F}}$ tomando índices sobre $\sigma$ de la siguiente manera:

$$
M_{\mathcal{F}}^{\sigma}:=\sum_{i \in \sigma}\left|\left\langle x, f_{i}\right\rangle\right|^{2}
$$

- si tenemos un par woven $(\mathcal{F}, \mathcal{G})$, denotaremos a sus cotas uniformes por $A_{\mathcal{F G}} \mathrm{y}$ $B_{\mathcal{F G}}$.

Proposición 3.5.2. Sean $\mathcal{F}, \mathcal{G}$ y $\mathcal{J}$ marcos para un espacio de Hilbert $\mathcal{H}$ tales que $(\mathcal{F}, \mathcal{G})$ y $(\mathcal{G}, \mathcal{J})$ son pares woven con cotas uniformes $A_{\mathcal{F G}}, B_{\mathcal{F G}}$ y $A_{\mathcal{F} \mathcal{G}}, B_{\mathcal{G} \mathcal{J}}$, respectivamente. Supongamos que se verifica que

$$
A_{\mathcal{G}} \leq B_{\mathcal{F G}}+B_{\mathcal{G J}} \text { y } B_{\mathcal{G}} \leq A_{\mathcal{F G}}+A_{\mathcal{G} \mathcal{J}}
$$

entonces $(\mathcal{F}, \mathcal{J})$ es un par woven.

Demostración. Sea $\sigma \subset I$ y $x \in \mathcal{H}$, ya que $(\mathcal{F}, \mathcal{G})$ y $(\mathcal{G}, \mathcal{J})$ es un par woven:

$$
A_{\mathcal{F G}}\|x\|^{2} \leq M_{\mathcal{F}}^{\sigma}+M_{\mathcal{G}}^{\sigma^{c}} \leq B_{\mathcal{F G}}\|x\|^{2} \text { y } A_{\mathcal{G} \mathcal{J}}\|x\|^{2} \leq M_{\mathcal{J}}^{\sigma^{c}}+M_{\mathcal{G}}^{\sigma} \leq B_{\mathcal{G} \mathcal{J}}\|x\|^{2} .
$$

Notar que con la notación definida, $M_{\mathcal{G}}^{\sigma}+M_{\mathcal{G}}^{\sigma^{c}}=M_{\mathcal{G}}$. Entonces

$$
\left(A_{\mathcal{F G}}+A_{\mathcal{G J}}\right)\|x\|^{2} \leq M_{\mathcal{F}}^{\sigma}+M_{\mathcal{J}}^{\sigma^{c}}+M_{\mathcal{G}} \leq\left(B_{\mathcal{F G}}+B_{\mathcal{G} \mathcal{J}}\right)\|x\|^{2} .
$$

Como $\mathcal{G}$ es marco,

$$
\left(A_{\mathcal{F G}}+A_{\mathcal{G} \mathcal{J}}-B_{\mathcal{G}}\right)\|x\|^{2} \leq M_{\mathcal{F}}^{\sigma}+M_{\mathcal{J}}^{\sigma^{c}} \leq\left(B_{\mathcal{F G}}+B_{\mathcal{G J}}-A_{\mathcal{G}}\right)\|x\|^{2}
$$

Por lo tanto, $(\mathcal{F}, \mathcal{J})$ es un par woven. 



\section{Capítulo 4}

\section{Perturbación de marcos de fusión}

Los marcos de subespacios fueron introducidos por Casazza y Kutyniok en [29] y desarrollados en [31] junto con S. Li bajo el nombre de marcos de fusión. La construcción de una noción general que incluyera a los marcos de vectores ya resultaba de interés antes de estos trabajos. En [57], M. Fornasier propone de forma constructiva marcos locales (para subespacios) que luego une, obteniendo un marco global (para el espacio total). En uno de sus lemas, Fornasier considera una familia de subespacios cerrados $\left\{W_{i}\right\}_{i \in I}$ de un espacio de Hilbert $\mathcal{H}$ y se refiere a sus proyecciones ortogonales $\left\{P_{W_{i}}\right\}_{i \in I}$ como proyecciones canónicas estables si verifican la siguiente desigualdad para todo $f \in \mathcal{H}$ y para cierto $N \in \mathbb{N}$ :

$$
\|f\|^{2} \leq \sum_{j \in \mathbb{Z}}\left\|P_{W_{i}}(f)\right\|^{2} \leq N\|f\|^{2}
$$

En la prueba de este lema, Fornasier agradece a Casazza por una sugerencia que simplifica su demostración. Un enfoque similar orientado a wavelets desarrollan A. Aldroubi, C. Cabrelli y U. Molter en [1]. Luego de los trabajos de Casazza y Kutyniok, surgieron generalizaciones de marcos de fusión entre las que podemos encontrar los g-marcos de Sun [98] y los K-g-marcos de fusión de Y. Huang y Y. Yang [74].

La generalización de marcos de vectores a subespacios resulta de gran utilidad en las aplicaciones. En el contexto de procesamiento de señales, cuando se trabaja con marcos de vectores, la señal se representa como una sucesión de escalares. Esto es, si $f$ corresponde a una señal del espacio $\mathcal{H}$ y $\left\{f_{i}\right\}_{i \in I}$ es un marco para $\mathcal{H}$, se consideran los escalares $\left\{\left\langle f, f_{i}\right\rangle\right\}_{i \in I}$ que miden las amplitudes de las proyecciones de la señal sobre cada vector $f_{i}$. Por otro lado, si se utilizan marcos de subespacios, la señal se representa con las proyecciones sobre cada subespacio: si $\left\{\left(W_{i}, w_{i}\right)\right\}_{i \in I}$ es un marco de subespacios con operador de marco $S_{\mathcal{W}}$, la señal se puede reconstruir a partir de sus medidas $\left\{w_{i}^{2} P_{W_{i}} f\right\}_{i \in I}$ :

$$
f=\sum_{i \in I} w_{i}^{2} S_{\mathcal{W}}^{-1}\left(P_{W_{i}} f\right)
$$

De esta manera se puede tratar a la señal en cada subespacio de forma independiente, 
lo que resulta más eficiente que el enfoque vectorial.

De los problemas que se pueden abordar dentro de esta clase de marcos nos centramos en el problema de perturbación de marcos de fusión por operadores. Este problema, como muchos otros, fue estudiado con anterioridad para vectores: dado un marco $\mathcal{F}=\left\{f_{i}\right\}_{i \in I}$ para un espacio de Hilbert $\mathcal{H}$ y un operador $T \in L(\mathcal{H})$, se buscan condiciones sobre $T$ de forma que $\left\{T f_{i}\right\}_{i \in I}$ sea también un marco para $\mathcal{H}$. En el contexto de marcos de fusión resulta más complejo que su versión vectorial ya que, entre las hipótesis necesarias, el operador de perturbación debe satisfacer que los subespacios perturbados resulten cerrados en $\mathcal{H}$.

En este capítulo realizamos un estudio de condiciones de suficiencia para que un marco de fusión conserve sus cualidades de marco bajo la perturbación por un operador. Adicionalmente, extendemos los pesos que se pueden considerar para el marco de fusión perturbado, presentando un rango de pesos admisibles. Estos resultados se encuentran publicados en [24].

Para facilitar la lectura consideraremos en este capítulo la siguiente definición:

Definición 4.0.1. Sea $\mathcal{F}$ una sucesión marco (marco, marco de fusión o sucesión marco de fusión) para $\mathcal{H}$ y $T \in L(\mathcal{H}, \mathcal{K})$. Decimos que $T$ es una perturbación efectiva de $\mathcal{F}$ si $T(\mathcal{F})$ es una sucesión marco para $\mathcal{K}$ (marco, marco de fusión o sucesión marco, respectivamente).

\subsection{Perturbación de marcos vectoriales}

En esta sección revisamos los resultados clásicos de perturbación de marcos vectoriales. A medida que avance el capítulo, se podrá apreciar cuáles de estos resultados se pueden llevar a marcos de fusión.

\subsubsection{Bases y sucesiones de Bessel}

El estudio de perturbación de sucesiones es previo al contexto de marcos. En general, el interés de este tipo de problemas es caracterizar aquellas perturbaciones que se encuentran cercanas, en algún sentido, a una sucesión que tenga alguna propiedad.

Uno de los resultados clásicos de este tipo remite a Paley y Wiener en 1934: si $\left\{f_{k}\right\}_{k=1}^{\infty}$ es una base para un espacio de Banach $X$, entonces la sucesión $\left\{g_{k}\right\}_{k=1}^{\infty}$ en $X$ es también una base si existe una constante $\lambda \in(0,1)$ tal que

$$
\left\|\sum_{k=1}^{\infty} c_{k}\left(f_{k}-g_{k}\right)\right\| \leq \lambda\left\|\sum_{k=1}^{\infty} c_{k} f_{k}\right\|,
$$

para toda sucesión $\left\{c_{k}\right\}_{k=1}^{\infty}$ con finitas coordenadas no nulas. El teorema de PaleyWiener resulta de utilidad para mostrar cuándo una sucesión es una base de Riesz para un espacio de Hilbert, por lo que se lo puede encontrar en el contexto de análisis wavelet. 
En el caso que la sucesión a perturbar sea una sucesión de Bessel, (4.1) se puede expresar en términos de un operador

$$
K: \ell^{2}(\mathbb{N}) \rightarrow \mathcal{H}, K\left\{c_{k}\right\}_{k=1}^{\infty}=\sum_{k=1}^{\infty} c_{k}\left(f_{k}-g_{k}\right) .
$$

En la siguiente sección daremos más especificaciones de este operador.

\subsubsection{Marcos de vectores}

Recopilamos algunos de los resultados de perturbación de marcos vectoriales, dicho estudio se debe principalmente a Christensen. El último de los resultados de esta sección es una adaptación de una de sus hipótesis a sucesiones marco utilizando ángulos entre subespacios.

Si se considera un marco para $\mathcal{H}$ y el operador $K$ definido en (4.2) con una adecuada acotación, la sucesión perturbada resulta también un marco.

Teorema 4.1.1. [36] Sea $\mathcal{F}=\left\{f_{k}\right\}_{k=1}^{\infty}$ un marco para un espacio Hilbert $\mathcal{H}$ con cotas de marcos $A_{\mathcal{F}}, B_{\mathcal{F}} y$ sean $\lambda, \mu \geq 0$ tales que $\lambda+\frac{\mu}{\sqrt{A}}<1 y$

$$
\left\|K\left\{c_{k}\right\}_{k=1}^{\infty}\right\| \leq \lambda\left\|\sum_{k=1}^{\infty} c_{k} f_{k}\right\|+\mu\left(\sum_{k=1}^{\infty}\left|c_{k}\right|^{2}\right)^{1 / 2}
$$

para toda sucesión finita $\left\{c_{k}\right\}_{k=1}^{\infty}$. Entonces $\left\{g_{k}\right\}_{k=1}^{\infty}$ es un marco para un espacio Hilbert $\mathcal{H}$ con cotas de marcos

$$
A\left(1-\left(\lambda+\frac{\mu}{\sqrt{A}}\right)\right)^{2}, B\left(1+\lambda+\frac{\mu}{\sqrt{B}}\right)^{2} .
$$

En [39], Christensen y Heil agregaron la hipótesis de compacidad al operador $K$ y probaron un condición de perturbación de sucesiones marco.

Teorema 4.1.2. Sea $\mathcal{F}=\left\{f_{k}\right\}_{k=1}^{\infty}$ un marco para un espacio Hilbert $\mathcal{H}$ y $\left\{g_{k}\right\}_{k=1}^{\infty}$ una sucesión en $\mathcal{H}$. Si

$$
K: \ell^{2}(\mathbb{N}) \rightarrow \mathcal{H}, K\left\{c_{k}\right\}_{k=1}^{\infty}=\sum_{k=1}^{\infty} c_{k}\left(f_{k}-g_{k}\right) .
$$

está bien definido y es compacto, entonces $\left\{g_{k}\right\}_{k=1}^{\infty}$ es una sucesión marco.

Notemos que, si $\mathcal{F}$ y $\mathcal{G}$ son sucesiones de Bessel, se puede considerar al operador $K$ como la diferencia entre los operadores de síntesis de ambas sucesiones, esto es:

$$
K\left\{c_{k}\right\}_{k=1}^{\infty}=\sum_{k=1}^{\infty} c_{k}\left(f_{k}-g_{k}\right)=\left(T_{\mathcal{F}}-T_{\mathcal{G}}\right)\left\{c_{k}\right\}_{k=1}^{\infty}
$$


Más aún, si $K$ es un operador acotado, basta que ver $\mathcal{F}$ sea una sucesión de Bessel para que $\mathcal{G}$ también lo sea. Con lo cual, atacar el problema de perturbaciones de un marco se puede traducir en el estudio de perturbaciones de su operador de síntesis. Nos enfocaremos en el caso particular de aquellas perturbaciones obtenidas por operadores $A \in L(\mathcal{H})$ tales que $T_{\mathcal{G}}=A T_{\mathcal{F}}$. Notar que si $A$ es un operador inversible, $\mathcal{F}$ y $\mathcal{G}$ resultan ser lo que se conoce como marcos equivalentes. En este caso, es fácil ver que el marco perturbado por un operador inversible también es un marco:dado $x \in \mathcal{H}$,

$$
\sum_{k \in I}\left|\left\langle x, A f_{k}\right\rangle\right|^{2} \leq B_{\mathcal{F}}\left\|A^{*} x\right\|^{2} \leq B\|A\|^{2}\|x\|^{2}
$$

y para la cota inferior, como $x=\left(A^{*}\right)^{-1} A^{*} x$,

$$
\|x\|^{2} \leq\left\|\left(A^{*}\right)^{-1}\right\|^{2}\left\|A^{*} x\right\|^{2} \leq \frac{\left\|\left(A^{*}\right)^{-1}\right\|^{2}}{A_{\mathcal{F}}} \sum_{k \in I}\left|\left\langle A^{*} x, f_{k}\right\rangle\right|^{2},
$$

por lo que

$$
\frac{A_{\mathcal{F}}}{\left\|A^{*-1}\right\|^{2}}\|x\|^{2} \leq \sum_{k \in I}\left|\left\langle x, A f_{k}\right\rangle\right|^{2} .
$$

Ejemplo 4.1.3. Entre los marcos equivalentes a un marco dado $\mathcal{F}=\left\{f_{k}\right\}_{k \in \mathbb{N}}$, dos ejemplos conocidos de perturbaciones resultan ser el marco dual canónico $\left\{S_{\mathcal{F}}^{-1} f_{k}\right\}_{k \in \mathbb{N}}$ y el llamado marco de Parseval asociado $\left\{S_{\mathcal{F}}^{-1 / 2} f_{k}\right\}_{k \in \mathbb{N}}$.

Si bien no es imprescindible que una perturbación tenga que ser inversible para perturbar un marco de forma efectiva, en general, el espacio generado por el marco perturbado no corresponde con el Hilbert completo. Si, por ejemplo, la perturbación es una proyección ortogonal, el marco perturbado es una sucesión marco.

Proposición 4.1.4. Sea $\mathcal{F}=\left\{f_{k}\right\}_{k=1}^{\infty}$ un marco para un espacio Hilbert $\mathcal{H}$ con cotas $A_{\mathcal{F}}$ y $B_{\mathcal{F}}$ y $V$ un subespacio cerrado para $\mathcal{H}$, entonces $\left\{P_{V} f_{k}\right\}_{k=1}^{\infty}$ es un marco para $V$ con cotas $A_{\mathcal{F}}$ y $B_{\mathcal{F}}$.

En general,

Proposición 4.1.5. [34] Sea $\mathcal{F}=\left\{f_{k}\right\}_{k=1}^{\infty}$ un marco para un espacio Hilbert $\mathcal{H}$ con cotas $A_{\mathcal{F}}, B_{\mathcal{F}}$ y sea $T \in L(\mathcal{H})$ un operador de rango cerrado. Entonces $\left\{T f_{k}\right\}_{k=1}^{\infty}$ es un marco para $R(T)$ con cotas $A_{\mathcal{F}}\left\|T^{\dagger}\right\|, B_{\mathcal{F}}\|T\|^{2}$.

Este resultado no puede aplicarse directamente a sucesiones marco, es decir, que el operador de perturbación posea rango cerrado no es suficiente para que sea una perturbación efectiva. Lo vemos en el siguiente ejemplo.

Ejemplo 4.1.6. Si $\left\{e_{k}\right\}_{k=1}^{\infty}$ es una base ortonormal para $\mathcal{H}$, definamos un operador $U: \mathcal{H} \rightarrow \mathcal{H}$ por

$$
U e_{2 k}=e_{2 k}, U e_{2 k-1}=\frac{1}{k} e_{2 k}, k \in \mathbb{N}
$$


$U$ resulta de rango cerrado. Es claro que tomar los elementos impares de la base ortonormal $\left\{e_{2 k-1}\right\}_{k=1}^{\infty}$ es una sucesión marco, sin embargo, $U$ no resulta una perturbación efectiva de esta sucesión marco. Si tomamos una sucesión $\left(x_{j}\right)_{j \in \mathbb{N}} \in$ $\overline{\operatorname{span}}_{k \in \mathbb{N}}\left\{U e_{2 k-1}\right\}$, dada por $x_{j}=e_{2 j}$, tenemos para cada $j \in \mathbb{N}$ que

$$
\sum_{k \in I}\left|\left\langle x_{j}, U e_{2 k-1}\right\rangle\right|^{2}=\sum_{k \in I}\left|\left\langle e_{2 j}, \frac{1}{k} e_{2 k}\right\rangle\right|^{2} \leq \frac{1}{j^{2}} \underset{j \rightarrow \infty}{\longrightarrow} 0 .
$$

Concluimos esta sección con una condición de ángulo que permite aplicar la hipótesis de rango cerrado de Christensen para perturbar sucesiones marco de forma efectiva. Recordemos que para subespacios cerrados $\mathcal{M}, \mathcal{N}$ de $\mathcal{H}$, el seno del ángulo se definía como $s(\mathcal{M}, \mathcal{N})=\left(1-c(\mathcal{M}, \mathcal{N})^{2}\right)^{1 / 2}$.

Proposición 4.1.7. Sea $\mathcal{F}=\left\{f_{k}\right\}_{k=1}^{\infty}$ una sucesión marco para un espacio de Hilbert $\mathcal{H}$ con operador de sintesis $T_{\mathcal{F}}$ y sea $\mathcal{M}:=\overline{\operatorname{span}}_{i \in I}\left\{f_{i}\right\}$. Sea $T \in L(\mathcal{H})$ un operador de rango cerrado tal que

$$
0<s(N(T), \mathcal{M})
$$

entonces $\left\{T f_{k}\right\}_{k=1}^{\infty}$ es una sucesión marco para $\mathcal{H}$.

Demostración. Para que $\left\{T f_{k}\right\}_{k=1}^{\infty}$ sea una sucesión marco para $\mathcal{H}$ basta ver que $T T_{\mathcal{F}}$ tiene rango cerrado (Proposición 1.3.2). Como $T$ y $T_{\mathcal{F}}$ son operadores de rango cerrado, por la Proposición 1.1.3, $\gamma(T)$ y $\gamma\left(T_{\mathcal{F}}\right)$ son positivos. Luego, por Proposición 1.1.4,

$$
\gamma(T) \gamma\left(T_{\mathcal{F}}\right) s(N(T), \mathcal{M}) \leq \gamma\left(T T_{\mathcal{F}}\right) \leq\|T\|\left\|T_{\mathcal{F}}\right\| s(N(T), \mathcal{M})
$$

Se tiene entonces que $\gamma\left(T T_{\mathcal{F}}\right)>0$, por lo que $T T_{\mathcal{F}}$ tiene rango cerrado.

\subsection{Perturbación de marcos de fusión}

En la sección anterior vimos que si el operador de perturbación $T \in L(\mathcal{H})$ lo consideramos de rango cerrado, aplicado a una sucesión marco de vectores, no necesariamente la sucesión perturbada será una sucesión marco. Algo similar ocurre para marcos de fusión, la hipótesis de rango cerrado tampoco es suficiente para que la perturbación sea efectiva, independientemente de los pesos elegidos. El siguiente ejemplo ilustra este hecho.

Ejemplo 4.2.1. Sea $B=\left\{e_{n}\right\}_{n \in \mathbb{N}}$ una base ortonormal de $\mathcal{H}$. Si consideramos el subespacio

$$
E_{k}=\operatorname{span}\left\{e_{2 k-1}, e_{2 k}\right\} \text { para } k \in \mathbb{N},
$$

la familia $\left\{E_{k}\right\}_{k \in \mathbb{N}}$ resulta una base ortonormal de subespacios para $\mathcal{H}$. Sea $T: \mathcal{H} \rightarrow \mathcal{H}$ 
el operador definido en un denso por

$$
T e_{n}=\left\{\begin{array}{lll}
2^{-k} e_{1} & \text { si } n=2 k-1 \\
e_{k+1} & \text { si } n=2 k
\end{array}\right.
$$

Es claro que la sucesión $\left\{T e_{k}\right\}_{k \in \mathbb{N}}$ es un marco para $\mathcal{H}$, por lo que podemos extender $T$ a un operador acotado y suryectivo. Veamos que la sucesión de subespacios cerrados

$$
W=\left\{W_{k}\right\}_{k \in \mathbb{N}} \text { con } W_{k}=T\left(E_{k}\right)=\operatorname{span}\left\{e_{1}, e_{k+1}\right\}
$$

no admite ningún peso $w$ que haga de $\mathcal{W}_{w}$ un marco de fusión. Por el contrario, supongamos que existe algún peso $w=\left\{w_{i}\right\}_{i \in I} \in \ell_{+}^{\infty}(I)$ para el cual esto sí sucede. Aplicando la desigualdad de la definición de marco de fusión a $f=e_{1}$, como $f \in$ $\bigcap_{k \in \mathbb{N}} W_{k}$ tenemos que $w \in \ell^{2}(\mathbb{N})$. Con lo cual, para todo $k \in \mathbb{N}$,

$$
A_{\mathcal{W}_{w}}=A_{\mathcal{W}_{w}}\left\|e_{k+1}\right\|^{2} \leq \sum_{j \in \mathbb{N}} w_{j}^{2}\left\|P_{W_{j}} e_{k+1}\right\|^{2}=w_{k}^{2} \underset{k \rightarrow \infty}{\longrightarrow} 0
$$

lo que contradice la existencia de cota inferior de marco de fusión.

\subsubsection{Antecedentes de perturbación}

Uno de los primeros resultados de perturbación de marcos de fusión surge de la necesidad de probar que, dado un marco de fusión $\left\{\left(W_{i}, w_{i}\right)\right\}_{i=1}^{\infty}$ con operador de marco $S_{\mathcal{W}_{w}},\left\{\left(S_{\mathcal{W}_{w}}^{-1} W_{i}, w_{i}\right)\right\}_{i=1}^{\infty}$ es también otro marco de fusión. Notar la analogía con el marco dual canónico en el caso de marcos vectoriales (Ejemplo 4.1.3). Para probar esto, Casazza y Kutyniok asumieron que una perturbación inversible de un marco $T$ resulta efectiva. En la prueba los autores usaron que

$$
P_{T\left(W_{i}\right)}=T P_{W_{i}} T^{-1}, i \in I \text {. }
$$

P. Gavruta observó que esta igualdad tiene una imprecisión: $T P_{W_{i}} T^{-1}$ no resulta una proyección ortogonal a menos que

$$
T^{*} T\left(W_{i}\right) \subset W_{i}
$$

Para realizar una prueba correcta, Gavruta obtiene relaciones sobre las proyecciones ortogonales de subespacios perturbados.

Proposición 4.2.2. Sea $T \in L(H)$ y $W$ un subespacio cerrado de $\mathcal{H}$. Son equivalentes:

1. $P_{\overline{T(W)}} T=T P_{W}$;

2. $T^{*} T(W) \subset W$. 
Demostración. $(i) \Rightarrow(i i)$ Sea $h \in W^{\perp}$. Como

$$
P_{\overline{T(W)}} T h=T P_{W} h=0,
$$

se tiene que $T h \in \overline{T W}^{\perp}=(T(W))^{\perp}$. Luego

$\langle T h, T w\rangle=0$ para todo $w \in W$, o equivalentemente, $\left\langle h, T^{*} T w\right\rangle=0$ para todo $w \in W$.

Por lo tanto,

$$
W^{\perp} \subset\left(T^{*} T(W)\right)^{\perp}
$$

y tomando ortogonales obtenemos $(i i)$.

$($ ii $) \Rightarrow(i)$

Para $w \in W$ observar que

$$
P_{\overline{T(W)}} T w=T w \quad \text { y } T P_{W} w=T w
$$

Por otro lado, si $h \in W^{\perp}, T P_{W} h=T 0=0$. Por hipótesis, como en (4.4), tenemos que $h \in\left(T^{*} T(W)\right)^{\perp}$, lo que implica que $T h \in(\overline{T(W)})^{\perp}$. Luego, $P \overline{T(W)} T h=0$.

Ahora bien, dado $x \in \mathcal{H}$, si $x=w+h$, con $w \in W, h \in W^{\perp}$, tenemos que

$$
P_{\overline{T(W)}} T x=P_{\overline{T(W)}} T w+P_{\overline{T(W)}} T h=T w=T P_{W} w+T P_{W} h=T P_{W} x .
$$

Lema 4.2.3. Sea $T \in L(\mathcal{H})$ y $W$ un subespacio cerrado de $\mathcal{H}$. Entonces

$$
P_{W} T^{*}=P_{W} T^{*} P_{\overline{T(W)}} .
$$

Demostración. Si $f \in \mathcal{H}$, entonces

$$
f=P_{\overline{T(W)}} f+g, g \in \overline{T(W)}^{\perp}=(T(W))^{\perp} .
$$

Por lo que,

$$
T^{*} f=T^{*} P_{\overline{T(W) f}}+T^{*} g .
$$

Para $w \in W$ se tiene que

$$
\left\langle T^{*} g, w\right\rangle=\langle g, T w\rangle=0,
$$

entonces $T^{*} g \in W^{\perp}$. Por lo tanto,

$$
P_{W} T^{*} f=P_{W} T^{*} P_{\overline{T(W)}} f+P_{W} T^{*} g=P_{W} T^{*} P_{\overline{T(W)}} f .
$$

Estos dos resultados permitieron a Gavruta estudiar la desigualdad marco de la sucesión $\left\{\overline{T\left(W_{i}\right)}\right\}_{i \in I}$, intercambiando cuidadosamente el operador inversible con las 
proyecciones a los subespacios, dando lugar al siguiente teorema:

Teorema 4.2.4. [60] Sea $\left\{\left(W_{i}, w_{i}\right)\right\}_{i=1}^{\infty}$ un marco de fusión de $\mathcal{H}$ con cotas $A_{\mathcal{W}_{w}} y$ $B_{\mathcal{W}_{w}}$ y $T \in L(\mathcal{H})$ un operador inversible, entonces $\left(\overline{T\left(W_{i}\right)}, w_{i}\right)_{i=1}^{\infty}$ es un marco de fusión con cotas.

$$
\frac{A_{\mathcal{W}_{w}}}{\left\|T^{*}\right\|^{2} B_{\mathcal{W}_{w}}} \text { y }\left\|T^{*}\right\|^{2}\left\|\left(T^{*}\right)^{-1}\right\|^{2}
$$

M. Asgari también consideró la clausura de los subespacios pero, en cambio, el operador $T$ debía ser suryectivo junto con la condición (4.3) sobre los subespacios [6].

Teorema 4.2.5. (Asgari) Sea $\left\{\left(W_{i}, w_{i}\right)\right\}_{i=1}^{\infty}$ un marco de fusión de $\mathcal{H}, T \in L(\mathcal{H})$ suryectivo tal que $T^{*} T\left(W_{i}\right) \subset W_{i}$ para todo $i$, entonces $\left(\overline{T\left(W_{i}\right)}, w_{i}\right)_{i=1}^{\infty}$ es un marco de fusión.

Por su parte, X. Li, L. Yang e Y. Zhu generalizaron la condición de invarianza de Asgari en términos del gap [85]:

Teorema 4.2.6. Sea $T \in L(\mathcal{H}, \mathcal{K})$ un operador de rango cerrado y $\left\{\left(W_{i}, w_{i}\right)\right\}_{i=1}^{\infty}$ un marco de fusión de $\mathcal{H}$. Las siguientes condiciones implican que $\left\{\left(\overline{T\left(W_{i}\right)}, w_{i}\right)\right\}_{i=1}^{\infty}$ es un marco de fusión para $\mathcal{K}$ :

1. $\sup _{i \in \mathbb{Z}^{+}} \delta\left(\overline{T^{*} T\left(W_{i}\right)}, W_{i}\right)<1$.

2. $\sup _{i \in \mathbb{Z}^{+}} \delta\left(\overline{T^{\dagger} T\left(W_{i}\right)}, W_{i}\right)<1$.

3. Existe $N \geq 2$ tal que $\left(T^{*} T\right)^{N}\left(W_{i}\right) \subset W_{i}$ para todo $i \in \mathbb{N}$.

Notar que en el caso que $T^{*} T\left(W_{i}\right) \subset W_{i}$, como pide el Teorema 4.2.5, trivialmente implica $\delta\left(\overline{T^{*} T\left(W_{i}\right)}, W_{i}\right)=0$. También el item 2. se verifica trivialmente con $N=1$ en este caso.

Considerando la primer desigualdad del Teorema 4.2.8, las hipótesis del teorema de Li et al. aseguran que los subespacios perturbados son cerrados, por lo que en el enunciado del teorema se podría prescindir de la clausura de $T\left(W_{i}\right)$.

El siguiente resultado se debe a Ruiz y Stojanoff [97]. Lo enunciamos para operadores de rango cerrado, en lugar de operadores suryectivos como hicieron los autores. La prueba sigue las mismas líneas de [97].

Teorema 4.2.7. Sea $\mathcal{E}=\left\{E_{i}\right\}_{i \in I}$ una base ortonormal de subespacios para un espacio de Hilbert $\mathcal{K}$ y sea $T \in L(\mathcal{H}, \mathcal{K})$ un operador de rango cerrado. Supongamos que

$$
c:=\inf _{i \in I} \frac{\gamma\left(T P_{E_{i}}\right)^{2}}{\left\|T P_{E_{i}}\right\|^{2}}>0
$$

y sean $0<A \leq B$ tales que $\frac{A}{B} \leq c$. Sea $w=\left\{w_{i}\right\}_{i \in I} \in \ell_{+}^{\infty}(I)$ tal que

$$
\frac{\left\|T P_{E_{i}}\right\|^{2}}{B} \leq w_{i}^{2} \leq \frac{\gamma\left(T P_{E_{i}}\right)^{2}}{A} \text { para todo } i \in I \text {. }
$$

Entonces: 
1. La familia $T(\mathcal{E}):=\left(T\left(E_{i}\right), w_{i}\right)$ es una sucesión marco de fusión para $\mathcal{K}$.

2. Las cotas de marco de $T(\mathcal{E})$ verifican que

$$
\frac{\gamma(T)^{2}}{B} \leq A_{T(\mathcal{E})} \leq \frac{\gamma(T)^{2}}{A} \text { y } \frac{\|T\|^{2}}{B} \leq B_{T(\mathcal{E})} \leq \frac{\|T\|^{2}}{A}
$$

Notemos que la hipótesis de uniformidad de las cotas nos permiten afirmar que cada subespacio perturbado es cerrado en $\mathcal{K}$.

\subsection{2. $\quad$ El gap de subespacios cerrados perturbados}

En esta sección hallamos condiciones sobre el operador de perturbación en términos del gap. Esto nos permitirá asegurar que las pertubaciones de la familia de subespacios de un marco de fusión sean, a su vez, subespacios cerrados. El ángulo entre subespacios y sus propiedades serán de gran utilidad.

Teorema 4.2.8. Sea $A \in L(\mathcal{H})^{+}$un operador de rango cerrado y $\mathcal{M}$ un subespacio cerrado de $\mathcal{H}$ tales que $c(N(A), \mathcal{M})<1$. Entonces se tienen las siguientes desigualdades

$$
c(N(A), \mathcal{M}) \leq c\left(A^{-1}\left(\mathcal{M}^{\perp}\right), \mathcal{M}\right) \leq\left[1-\frac{\gamma\left(A^{1 / 2}\right)^{4}}{\|A\|^{2}}\left(1-c(N(A), \mathcal{M})^{2}\right)\right]^{1 / 2} .
$$

Demostración. Notemos que

$$
A^{-1}\left(\mathcal{M}^{\perp}\right) \cap \mathcal{M}=N(A) \cap \mathcal{M}
$$

ya que, si $x \in \mathcal{M}$ con $A x \in \mathcal{M}^{\perp}$,

$$
0=\langle A x, x\rangle=\left\langle A^{1 / 2} x, A^{1 / 2} x\right\rangle=\left\|A^{1 / 2} x\right\|,
$$

por lo que $A x=A^{1 / 2}\left(A^{1 / 2} x\right)=0$. Luego, como $N(A) \subset A^{-1}\left(\mathcal{M}^{\perp}\right)$ tenemos que

$$
N(A) \ominus(N(A) \cap \mathcal{M}) \subset A^{-1}\left(\mathcal{M}^{\perp}\right) \ominus\left(A^{-1}\left(\mathcal{M}^{\perp}\right) \cap \mathcal{M}\right) .
$$

Con estas consideraciones, la primera desigualdad se deduce de la definición de ángulo.

Por otro lado, observar que $A^{1 / 2} P_{\mathcal{M}}$ tiene rango cerrado, ya que $N\left(A^{1 / 2}\right)=N(A)$ y $c(N(A), \mathcal{M})<1$. Entonces, por la Proposición 1.1.4,

$$
\gamma\left(P_{\mathcal{M}} A P_{\mathcal{M}}\right)=\gamma\left(A^{1 / 2} P_{\mathcal{M}}\right)^{2} \geq \gamma\left(A^{1 / 2}\right)^{2}\left(1-c(N(A), \mathcal{M})^{2}\right)
$$

Dado que $N\left(P_{\mathcal{M}} A\right)=A^{-1}\left(\mathcal{M}^{\perp}\right)$, nuevamente por la Proposición 1.1.4 tenemos 
que

$$
\begin{aligned}
\gamma\left(P_{\mathcal{M}} A P_{\mathcal{M}}\right) & \leq\left\|P_{\mathcal{M}} A\right\|\left(1-c\left(A^{-1}\left(\mathcal{M}^{\perp}\right), \mathcal{M}\right)^{2}\right)^{1 / 2} \\
& \leq\|A\|\left(1-c\left(A^{-1}\left(\mathcal{M}^{\perp}\right), \mathcal{M}\right)^{2}\right)^{1 / 2}
\end{aligned}
$$

Combinando (4.5) y (4.6) deducimos que

$$
c\left(A^{-1}\left(\mathcal{M}^{\perp}\right), \mathcal{M}\right) \leq\left[1-\frac{\gamma\left(A^{1 / 2}\right)^{4}}{\|A\|^{2}}\left(1-c(N(A), \mathcal{M})^{2}\right)\right]^{1 / 2} .
$$

El teorema nos proporciona una serie de condiciones equivalentes:

Corolario 4.2.9. Sea $A \in L(\mathcal{H})^{+}$un operador rango cerrado y $\left\{\mathcal{M}_{i}\right\}_{i \in I}$ una sucesión de subespacios cerrados de $\mathcal{H}$. Entonces, son equivalentes:

1. $\inf _{i \in I} \gamma\left(A P_{\mathcal{M}_{i}}\right)>0$.

2. $\sup _{i \in I} c\left(N(A), \mathcal{M}_{i}\right)<1$.

3. $\sup _{i \in I} \delta\left(\overline{A\left(\mathcal{M}_{i}\right)}, \mathcal{M}_{i}\right)<1$.

Demostración. La equivalencia entre 1. y 2. se deduce de la Proposición 1.1.4. Para probar que 2. y 3. son equivalentes usaremos el hecho que, tanto 2. y 3. como 1., implican que, para todo $i \in I$,

$$
\delta\left(\overline{A\left(\mathcal{M}_{i}\right)}, \mathcal{M}_{i}\right)=c\left(A^{-1}\left(\mathcal{M}_{i}^{\perp}\right), \mathcal{M}_{i}\right) .
$$

El resto de la prueba se concluye recurriendo al Teorema 4.2.8.

Si suponemos que $c\left(N(A), \mathcal{M}_{i}\right)<1$, tenemos que $A\left(\mathcal{M}_{i}\right)=\overline{A\left(\mathcal{M}_{i}\right)}$, lo que implica

$$
\begin{aligned}
\delta\left(\overline{A\left(\mathcal{M}_{i}\right)}, \mathcal{M}_{i}\right) & =\left\|P_{\mathcal{M}_{i}^{\perp}} P_{A\left(\mathcal{M}_{i}\right)}\right\| \\
& =\left\|P_{\mathcal{M}_{i}^{\perp}} P_{A\left(\mathcal{M}_{i}\right)} P_{\left(\mathcal{M}_{i}^{\perp} \cap A\left(\mathcal{M}_{i}\right)\right)^{\perp}}\right\| \\
& =c\left(\mathcal{M}_{i}^{\perp}, A\left(\mathcal{M}_{i}\right)\right)=c\left(\mathcal{M}_{i}, A^{-1}\left(\mathcal{M}_{i}^{\perp}\right)\right) \\
& =c\left(A^{-1}\left(\mathcal{M}_{i}^{\perp}\right), \mathcal{M}_{i}\right) .
\end{aligned}
$$

Por otro lado, si $\delta\left(\overline{A\left(\mathcal{M}_{i}\right)}, \mathcal{M}_{i}\right)<1$ entonces

$$
\overline{A\left(\mathcal{M}_{i}\right)} \cap \mathcal{M}_{i}^{\perp}=\{0\}
$$


Por lo tanto,

$$
\begin{aligned}
\delta\left(\overline{A\left(\mathcal{M}_{i}\right)}, \mathcal{M}_{i}\right)=\left\|P_{\mathcal{M}_{i}^{\perp}} P_{\overline{A\left(\mathcal{M}_{i}\right)}}\right\| & =c_{0}\left(\overline{A\left(\mathcal{M}_{i}\right)}, \mathcal{M}_{i}^{\perp}\right) \\
& =c\left(\overline{A\left(\mathcal{M}_{i}\right)}, \mathcal{M}_{i}^{\perp}\right) \\
& =c\left(\mathcal{M}_{i}, A^{-1}\left(\mathcal{M}_{i}^{\perp}\right)\right) .
\end{aligned}
$$

Notar que en el Teorema 4.2.8 no fue necesaria la hipótesis de la cota del ángulo para probar que

$$
c(N(A), \mathcal{M}) \leq c\left(A^{-1}\left(\mathcal{M}^{\perp}\right), \mathcal{M}\right) .
$$

Luego, se desprende del corolario que esta desigualdad nos dice que

$$
\delta(\overline{A(\mathcal{M})}, \mathcal{M})<1 \text { implica que } c\left(\mathcal{M}, A^{-1}\left(\mathcal{M}^{\perp}\right)\right)=\delta(\overline{A(\mathcal{M})}, \mathcal{M}) .
$$

En particular, esta cota del gap nos dice que $A(\mathcal{M})$ resulta un subespacio cerrado (Proposición 1.1.4).

\subsubsection{Variación de pesos}

Una de las características que distingue la perturbación de marcos de fusión respecto de los marcos vectoriales, además de las mencionadas, es la posibilidad de variar los pesos. Lo que permite considerar más de un marco de fusión a partir de una familia de subespacios. En esta sección consideramos la condición provista por el Teorema 4.2.7 para tener perturbaciones efectivas de sucesiones marcos de fusión y también cotas de los pesos de la familia perturbada. Además, relacionamos esta condición con la condición de gap de Li et al.

Teorema 4.2.10. Sea $\mathcal{W}_{w}=\left(W_{i}, w_{i}\right)_{i \in I}$ un marco de fusión para $\mathcal{H}, T \in L(\mathcal{H}, \mathcal{K})$ un operador de rango cerrado y tal que

$$
0<c:=\inf _{i \in I} \frac{\gamma\left(T P_{W_{i}}\right)^{2}}{\left\|T P_{W_{i}}\right\|^{2}}<1
$$

Sea $v=\left\{v_{i}\right\}_{i \in I} \in \ell_{+}^{\infty}(I)$ con cotas

$$
\frac{w_{i}^{2}\left\|T P_{W_{i}}\right\|^{2}}{\|T\|} \leq v_{i}^{2} \leq \frac{w_{i}^{2} \gamma\left(T P_{W_{i}}\right)^{2}}{\gamma(T)\left(1-c^{2}\right)^{1 / 2}} \text { para toda } i \in I,
$$

entonces $T(\mathcal{W}):=\left(T\left(W_{i}\right), v_{i}\right)_{i \in I}$ es una sucesión marco de fusión en $\mathcal{K}$.

Demostración. Si denotamos por $E_{j}$ a la copia isométrica de $W_{j}$ en $\mathcal{K}_{\mathcal{W}}=\bigoplus_{i \in I} W_{i}$, tenemos que $\left\{E_{j}\right\}_{j \in J}$ es una base ortonormal de subespacios de $\mathcal{K}_{\mathcal{W}}$. Consideremos 
la composición del operador de síntesis $T_{\mathcal{W}_{w}}$ con $T$ :

$$
J(g):=T T_{\mathcal{W}_{w}}(g)=\sum_{i \in I} w_{i} T\left(g_{i}\right), g \in \mathcal{K}_{\mathcal{W}}
$$

Es claro que

$$
\left\|J P_{E_{i}}\right\|=w_{i}\left\|T P_{W_{i}}\right\| \leq w_{i}\|T\|, \text { para todo } i \in I .
$$

Probamos una observación para la continuación de la prueba.

\section{Observación 4.2.11.}

$$
\gamma\left(J P_{E_{i}}\right)=w_{i} \gamma\left(T P_{W_{i}}\right), i \in I \text {. }
$$

Demostración. Dado que

$$
N\left(J P_{E_{i}}\right)^{\perp}=\left(\left(N(J) \cap E_{i}\right) \oplus E_{i}^{\perp}\right)^{\perp}=E_{i} \cap\left(N(J) \cap E_{i}\right)^{\perp}
$$

se tiene que todo vector unitario $x \in N\left(J P_{E_{i}}\right)^{\perp}$ es de la forma

$$
x=\left(0, \ldots, x_{i}, \ldots, 0\right) \text { con } x_{i} \in W_{i} \text { y }\left\|x_{i}\right\|=1 .
$$

Notar que $x_{i} \in\left(N(T) \cap W_{i}\right)^{\perp}$ : dado $y \in N(T) \cap W_{i}$, si tomamos el correspondiente $\tilde{y}$ perteneciente a $\mathcal{K}_{\mathcal{W}}, \tilde{y}=(0, \ldots, y, \ldots, 0) \in N(J) \cap E_{i}$. Por lo cual,

$$
\langle\tilde{y}, x\rangle=0 \Leftrightarrow\left\langle y, x_{i}\right\rangle=0 .
$$

Luego, tomando $x \in N\left(J P_{E_{i}}\right)^{\perp}$ con $\|x\|=1$,

$$
\left\|J P_{E_{i}} x\right\|=\left\|w_{i} T x_{i}\right\|=w_{i}\left\|T P_{W_{i}} x_{i}\right\|
$$

y se tiene que

$$
\gamma\left(J P_{E_{i}}\right) \geq w_{i} \gamma\left(T P_{W_{i}}\right)
$$

Las desigualdades restantes se pueden probar de una forma similar: tomamos $x \in\left(N(T) \cap W_{i}\right)^{\perp}$ y el correspondiente $\tilde{x}=(0, \ldots, x, \ldots, 0) \in E_{i}$. Nuevamente, $\tilde{x}$ pertenece a $\left(N(J) \cap E_{i}\right)^{\perp}$. Tenemos entonces que

$$
\left\|T P_{W_{i}} x\right\|=\frac{1}{w_{i}}\left\|w_{i} T x\right\|=\frac{1}{w_{i}}\|J \tilde{x}\| \geq \frac{1}{w_{i}} \gamma\left(J P_{E_{i}}\right) .
$$

Por (4.11) y (4.12), se tiene (4.10) por lo que se concluye la prueba esta observación.

Ahora bien, usando el lado derecho de (4.11) se tiene que

$$
\gamma\left(T P_{W_{i}}\right) \geq \gamma(T)\left(1-c\left(N(T), W_{i}\right)^{2}\right)^{1 / 2} \geq \gamma(T)\left(1-c^{2}\right)^{1 / 2}
$$


lo que completa la desigualdad

$$
\inf _{i \in I} \frac{\gamma\left(J P_{E_{i}}\right)}{\left\|J P_{E_{i}}\right\|} \geq \frac{\gamma(T)\left(1-c^{2}\right)^{1 / 2}}{\|T\|}>0
$$

Podemos entonces aplicar el teorema anterior. Consideremos las constantes

$$
A:=\gamma(T)^{2}\left(1-c^{2}\right) \text { y } B:=\|T\|^{2}
$$

y una familia de pesos $v=\left\{v_{i}\right\}_{i \in I} \in \ell_{+}^{\infty}(I)$ en (4.2.7):

$$
\frac{\left\|J P_{E_{i}}\right\|^{2}}{B} \leq v_{i}^{2} \leq \frac{\gamma\left(J P_{E_{i}}\right)^{2}}{A} \text { para todo } i \in I
$$

Usando (4.9), (4.10) y las constantes elegidas $A$ y $B$, tenemos que para todo $i \in I$,

$$
\frac{w_{i}^{2}\left\|T P_{W_{i}}\right\|^{2}}{\|T\|^{2}} \leq v_{i}^{2} \leq \frac{w_{i}^{2} \gamma\left(T P_{W_{i}}\right)^{2}}{\gamma(T)^{2}\left(1-c^{2}\right)} .
$$

Por el Teorema 4.2.7, estos pesos satisfacen que $T(\mathcal{W})$ es una sucesión marco de fusión para $\mathcal{K}$.

Observación 4.2.12. Supongamos que $\mathcal{W}_{w}$ es un marco de fusión para $\mathcal{H}$ y $T \in L(\mathcal{H})$ es un operador de rango cerrado. Entonces, usando el Corolario 4.2 .9 y el hecho que

$$
\left\|T P_{W_{i}}\right\| \leq\|T\|, \quad \text { para todo } i \in I,
$$

tenemos que cualquiera de las siguientes condiciones

1. $0<\inf _{i \in I} \gamma\left(T P_{W_{i}}\right)$

2. $\sup _{i \in I} c\left(N(T), W_{i}\right)<1$.

3. $\sup _{i \in I} \delta\left(\overline{T^{*} T\left(W_{i}\right)}, W_{i}\right)<1$

implican (4.8). En particular, el Teorema 4.2.10 generaliza las condiciones suficientes probadas en [85]. Por otro lado, es fácil construir ejemplos donde se verifica (4.8) pero $\inf _{i \in \mathcal{N}} \gamma\left(T P_{W_{i}}\right)=0$. En tal caso, el Teorema 4.2.10 implica que, considerando una familia diferente de pesos (que tienda a 0 ), la sucesión perturbada resulta una sucesión marco de fusión.

El siguiente es un ejemplo de una sucesión marco de fusión que se obtiene de perturbar un marco de fusión con un operador de rango cerrado y donde la (4.8) no resulta necesaria.

Ejemplo 4.2.13. Sea $\left\{e_{i}\right\}_{i \in \mathbb{N}}$ una base ortonormal para $\mathcal{H}$. Sea $\left\{\theta_{k}\right\}_{k=0}^{\infty}$ una sucesión de números positivos tales que $\theta_{0}<\frac{\pi}{4}$ con $\lim _{k \rightarrow \infty} \theta_{k}=0$. Para $k=0,1,2, \ldots$, consideramos

$$
E_{k}=\left\{e_{3 k+1}, \operatorname{sen}\left(\theta_{k}\right) e_{3 k+2}+\cos \left(\theta_{k}\right) e_{3(k+1)}\right\} \mathrm{y}
$$




$$
F_{k}=\operatorname{span}\left\{e_{3 k+1}, \frac{1}{\sqrt{2}} e_{3 k+2}+\frac{1}{\sqrt{2}} e_{3(k+1)}\right\} .
$$

La familia $\left\{W_{i}\right\}_{i \in \mathbb{N}}$ de subespacios cerrados de $\mathcal{H}$ dada por

$$
W_{2 k+1}=E_{k} \quad \mathrm{y} \quad W_{2(k+1)}=F_{k}
$$

forma un marco de fusión para $\mathcal{H}$ considerando los pesos $w_{i}=1$, para todo $i \in \mathbb{N}$. Este hecho se puede deducir observando que la unión de bases ortonormales que generan cada $E_{k}$ y $F_{k}$ generan un marco de vectores para $\mathcal{H}$. Más precisamente, para cada $k$, la familia de vectores dados por

$$
\begin{aligned}
& f_{1, k}=e_{3 k+1} \\
& f_{2, k}=\sin \left(\theta_{k}\right) e_{3 k+2}+\cos \left(\theta_{k}\right) e_{3(k+1)} \\
& f_{3, k}=e_{3 k+1} \\
& f_{4, k}=\frac{1}{\sqrt{2}} e_{3 k+2}+\frac{1}{\sqrt{2}} e_{3(k+1)}
\end{aligned}
$$

son un marco para $\operatorname{span}\left\{e_{3 k+1}, e_{3 k+2}, e_{3(k+1)}\right\}$, con cotas de marco

$$
A_{k}=1-\sqrt{\frac{3}{4}+\cos \left(\theta_{k}\right) \sin \left(\theta_{k}\right)} \text { y } \quad B_{k}=2 .
$$

Como las cotas inferiores están uniformemente acotadas por $0<1-\sqrt{\frac{3}{4}+\cos \left(\theta_{0}\right) \sin \left(\theta_{0}\right)}$ y los subespacios generados por estas sucesiones marco son mutuamente ortogonales, tenemos efectivamente un marco de fusión para $\mathcal{H}$.

Como operador perturbación consideremos a $T \in L(\mathcal{H})$ como la proyección ortonormal sobre

$$
\mathcal{K}=\overline{\operatorname{span}}\left\{e_{3 k+1} e_{3 k+2}, k=0,1, \ldots\right\} .
$$

Ya que $T\left(E_{k}\right)=T\left(F_{k}\right)=\operatorname{span}\left\{e_{3 k+1}, e_{3 k+2}\right\},\left(T\left(W_{i}\right), w_{i}\right)$ resulta una sucesión marco de fusión para $\mathcal{H}$. En contraposición a la condición (4.8), es fácil ver que

$$
\gamma\left(T P_{W_{i}}\right)= \begin{cases}\operatorname{sen}^{2}\left(\theta_{k}\right), & \text { si } i=2 k+1 \\ \frac{1}{2}, & \text { si } i=2(k+1)\end{cases}
$$

y $\left\|T P_{W_{i}}\right\|=1$ para todo $i \in \mathbb{N}$, por lo que ínf $i_{i \in \mathbb{N}} \frac{\gamma\left(T P_{W_{i}}\right)}{\left\|T P_{W_{i}}\right\|}=0$.

\subsection{Sobre perturbaciones efectivas de marcos de fu- sión RF}

Recordemos de la introducción de esta tesis que una sucesión vectorial $\mathcal{F}=\left\{f_{i}\right\}_{i=1}^{M}$ se dice que provee recuperación de fase $(\mathrm{RF})$ en $\mathcal{H}_{N}$, si para todo $x, y \in \mathcal{H}_{N}$ y para 
todo $i \in I$, la igualdad

$$
\left|\left\langle x, f_{i}\right\rangle\right|=\left|\left\langle y, f_{i}\right\rangle\right|,
$$

implica que existe un escalar $c$, con $|c|=1$, tal que $x=c y$.

Dada una sucesión vectorial $\mathcal{F}=\left\{f_{i}\right\}_{i=1}^{M}$ RF en $\mathcal{H}_{N}$, es claro que toda perturbación inversible $G$ resulta efectiva. En efecto, si para todo $x, y \in \mathcal{H}_{N}$ y para todo $i \in I$,

$$
\left|\left\langle x, G f_{i}\right\rangle\right|=\left|\left\langle y, G f_{i}\right\rangle\right|
$$

por ser $\mathcal{F}$ una sucesión RF, existe un escalar $c$ unitario, tal que $G^{*} x=c G^{*} y$. Aplicando $\left(G^{*}\right)^{-1}$ tenemos que $x=c y$.

Para sucesiones de subespacios, en cambio, no es tan sencillo verificar que una perturbación por un inversible provea RF por proyecciones. Esto se puede apreciar en la definición de RF para proyecciones:

Definición 4.3.1. Sea $\mathcal{W}=\left\{W_{i}\right\}_{i=1}^{N}$ una familia de subespacios de $\mathcal{H}_{M}$ con proyecciones ortogonales $P_{W_{i}}$. Se dice que $\mathcal{W}$ permite recuperación de fase si para todo $x, y \in \mathcal{H}_{M}$ y para todo $i \in I$,

$$
\left\|P_{W_{i}} x\right\|=\left\|P_{W_{i}} y\right\|
$$

implica que $x=$ cy para algún c unitario.

Como estudiamos anteriormente, existen operadores que, bajo ciertas condiciones, resultan ser perturbaciones efectivas para los marcos de fusión. Por ejemplo, si $\left(W_{i}, w_{i}\right)_{i \in I}$ es un marco de fusión y $G$ es un operador de rango cerrado, entonces $\left(G\left(W_{i}\right), w_{i}\right)_{i \in I}$ es un marco de fusión. En general, el problema de perturbación se torna más complejo al llevarlo al contexto de RF. A saber, si $\left(G\left(W_{i}\right), w_{i}\right)_{i \in I}$ es el marco de fusión perturbado, tendríamos como hipótesis inicial (4.13) para las proyecciones $P_{G\left(W_{i}\right)}$, las cuales no resultan operadores fáciles de tratar. Además, el hecho de ser un problema no lineal que demanda igualdad, impide acudir a herramientas de desigualdades conocidas.

Consideraremos un caso particular en donde podemos asegurar perturbación efectiva. Para los marcos de fusión los pesos que se consideran son $w_{i}=1$ para todo $i \in I$.

Lema 4.3.2. Sea $\left\{\left(W_{i}, w_{i}\right)\right\}_{i \in I}$ un marco de fusión y $U$ un operador unitario, entonces $\left\{\left(U\left(W_{i}\right), w_{i}\right)\right\}_{i \in I}$ es $R F$.

Demostración. Trivial ya que para todo $i \in I, P_{U\left(W_{i}\right)}=U P_{W_{i}} U^{*}$.

Para el caso particular de perturbaciones inversibles, presentamos una condición de conmutatividad que permite asegurar la efectividad de perturbaciones de marcos de fusión RF. 
Proposición 4.3.3. Sea $\mathcal{W}=\left\{\left(W_{i}, w_{i}\right)\right\}_{i \in I}$ un marco de fusión $R F$ y $G$ un operador inversible tal que para todo $i \in I$,

$$
G P_{W_{i}}=P_{W_{i}} G \text { y } G^{*} P_{W_{i}}=P_{W_{i}} G^{*}
$$

Entonces $\left(G\left(W_{i}\right), w_{i}\right)_{i \in I}$ es $R F$.

Demostración. Consideremos la descomposición polar de $G$,

$$
G=U A \text { siendo } A=|G|
$$

Utilizando cálculo funcional, como $G$ y $G^{*}$ conmutan con $P_{W_{i}}$,

$$
A P_{W_{i}}=P_{W_{i}} A
$$

Notemos que esto implica que $A\left(W_{i}\right) \subset W_{i}$, por lo que $A^{2}\left(W_{i}\right) \subset W_{i}$. En particular, por la Proposición 4.2.2, como $A\left(W_{i}\right)$ es cerrado por ser $A$ inversible, tenemos que

$$
P_{A\left(W_{i}\right)}=A P_{W_{i}} .
$$

Ahora bien, supongamos que para todo $i \in I$,

$$
\left\|P_{A\left(W_{i}\right)} x\right\|=\left\|P_{A\left(W_{i}\right)} y\right\| .
$$

Por (4.14), tenemos que

$$
\left\|A P_{W_{i}} x\right\|=\left\|A P_{W_{i}} y\right\|
$$

Por la hipótesis de conmutatividad

$$
\left\|P_{W_{i}} A x\right\|=\left\|P_{W_{i}} A y\right\|
$$

Entonces, por ser RF el marco de fusión y de la inversibilidad de $A,\left(A\left(W_{i}\right), w_{i}\right)_{i \in I}$ es RF. Luego, por el lema, $\left(G\left(W_{i}\right), w_{i}\right)_{i \in I}$ provee recuperación de fase.

Observación 4.3.4. Notar que, como consecuencia de la hipótesis sobre $G$, resulta que $G$ conmuta con el operador de marco del marco de fusión. 


\section{Capítulo 5}

\section{Extremos locales para problemas tipo Procusto}

Un problema de aproximación matricial, como dice N. Higham en [69], consiste en encontrar para una matriz arbitraria, el miembro más cercano de una clase de matrices dada, donde la distancia se mide con una norma matricial. Típicamente, dados $A \in M_{d}(\mathbb{C})$, una norma matricial $N(\cdot)$ en $M_{d}(\mathbb{C})$ y un conjunto de matrices $P \in M_{d}(\mathbb{C})$, se busca minimizar la distancia

$$
d_{N}(P, A)=\min \{N(A-X): X \in P\}
$$

Inspirados en el mito griego de Procusto, Hurley y Cattell (1962) denominaron como problemas de Procusto a una subclase de estos problemas. Esa denominación fue aceptada en la comunidad por lo que algunos autores dieron en llamar análisis de Procusto al estudio de estos problemas. Una formulación general de este problema es

$$
\min _{X \in P} N(A-B X)
$$

siendo $P$ un conjunto matricial con características prefijadas. Una de las primeras versiones de este problema que podemos destacar es el denominado problema doble de Procusto, planteado por Schönemann en 1968:

$$
\min _{X^{t} X=I} N\left(A-X^{*} B X\right), A, B \in M_{d}(\mathbb{C}) .
$$

En dicho problema resulta de utilidad considerar igualdades de trazas así como el orden de los autovalores en las descomposiciones espectrales [64]. Con el correr de los años, el problema de Procusto se ha ido diversificando, por lo que son numerosas las técnicas empleadas para su estudio, por lo que se lo puede considerar un problema que se adapta a distintos ambientes.

En análisis matricial, resulta natural el estudio de estructuras espectrales, lo que hace de la mayorización una herramienta fundamental. Para los problemas de aproximación, es útil conocer características de los espectros de las matrices involucradas. Es 
por esto que las desigualdades de Lidskii, en sus versiones aditivas y multiplicativas, cumplen un rol principal:

(Lidskii) Sean $A, B \in \mathcal{H}(d)$ con autovalores $\lambda(A), \lambda(B) \in\left(\mathbb{R}^{d}\right)^{\downarrow}$ entonces

$$
\lambda(A)^{\uparrow}+\lambda(B) \prec \lambda(A+B) \prec \lambda(A)+\lambda(B) .
$$

(Gelfand-Naimark-Lidskii) Sean $A, B \in \mathcal{P}(d)$, entonces

$$
\log (\lambda(B))+\log \left(\lambda^{\uparrow}(A)\right) \prec \log (\lambda(B A)) \prec \log (\lambda(B))+\log (\lambda(A)) .
$$

Dentro de las aplicaciones de la teoría de marcos, en el contexto de completación de marcos [90], estas desigualdades permiten obtener la completación óptima. Para esto, se construyen funciones a partir de la distancia a minimizar usando normas unitariamente invariantes y estrictamente convexas. Las desigualdades develan explícitamente a los minimizadores globales de estas funciones definidas en órbitas unitarias.

Recientemente, Bhatia y Congedo estudiaron en [9] un problema de Procusto tipo (5.1) en el conjunto de las matrices positivas con distintas métricas que se utilizan en teoría de la información. Para este problema, los autores muestran que el mejor aproximante a la órbita unitaria de cierta matriz tiene que conmutar con la matriz dada.

El objetivo de este capítulo es profundizar los resultados de Bhatia y Congedo basándonos en técnicas empleadas en [93] para el estudio local de desigualdades de Lidskii aditivas. Las primeras secciones están dedicadas a dar un panorama de los resultados clásicos de las desigualdades, mostrando algunas de las relaciones que se conocen en aplicaciones a problemas de diseño de marcos. Hacemos hincapié en los casos de igualdad de las desigualdades de Lidskii. Luego, presentamos las herramientas del caso aditivo que utilizaremos para nuestros resultados de una versión local de la desigualdad multiplicativa. En la última sección concentramos los resultados probados aplicándolos al problema de Procusto.

\subsection{Lidskii como un problema de optimalidad}

En esta sección vemos algunos de los resultados en donde las desigualdades se aplican en el estudio de óptimos espectrales de matrices. Nos basamos en aplicaciones a problemas de marcos para introducir de manera intuitiva algunos de los aspectos que resultan de interés. En particular, destacamos la relevancia de los casos de igualdad en estas desigualdades. Recordamos que denotamos por $\mathcal{P}(d)$ a las matrices definidas positivas. 


\subsubsection{Diseño de marcos}

Uno de los problemas de marcos que pueden ser abordados con herramientas de mayorización son los llamados problemas de diseño de marcos. En dichos problemas, el objetivo es buscar un marco que satisfaga características prefijadas. Un ejemplo clásico de este tipo de problemas está asociado al Teorema de Schur-Horn, donde los vectores del marco tienen normas predeterminadas [5]:

Proposición 5.1.1. Sea $B \in \mathcal{P}(d)$ con autovalores $\lambda^{\downarrow}=\left(\lambda_{i}\right)_{i=1}^{d}$ y sea $a=\left(\alpha_{i}\right)_{i=1}^{k}$ con $\alpha_{1} \geq \ldots \alpha_{k}>0$. Existe una sucesión $\mathcal{G}=\left\{g_{i}\right\}_{i=1}^{k}$ en $\mathcal{H}(d)$ con operador de marco $S_{\mathcal{G}}=B$ tal que $\left\|g_{i}\right\|^{2}=\alpha_{i}$ para todo $1 \leq i \leq k$ si y sólo si $a \prec \lambda$.

Posteriormente se incorporaron a estos problemas requisitos de optimalidad, esto es, se buscaba la sucesión óptima entre las que verificaban la condición de normas. Para medir la optimalidad, Benedetto y Fickus introdujeron en [7] la noción de potencial de marco (frame potential). Dada una sucesión $\left\{f_{i}\right\}_{i=1}^{M}$ de vectores unitarios en $\mathcal{H}_{N}$, el potencial de marco es

$$
F P\left(\left\{f_{i}\right\}_{i=1}^{M}\right)=\sum_{i, j=1}^{M}\left|\left\langle f_{i}, f_{j}\right\rangle\right|^{2}
$$

Benedetto y Fickus caracterizaron los marcos finitos ajustados normalizados en términos de los minimizadores del potencial. Mas aún, en la terminología del problema, probaron que los minimizadores locales también son globales. Estas ideas las desarrollamos en la siguiente sección.

En [90] Massey, Ruiz y Stojanoff, generalizaron el potencial de marco considerando los llamados potenciales convexos. Dado un marco $\left\{f_{i}\right\}_{i=1}^{M}$ y una función convexa $\phi:[0, \infty) \rightarrow[0, \infty)$, usando cálculo funcional se define el potencial convexo por

$$
P_{\phi}(\mathcal{F})=\operatorname{tr} \phi\left(S_{\mathcal{F}}\right)=\sum_{j=1}^{M} \phi\left(\lambda_{j}\left(S_{\mathcal{F}}\right)\right) \text { para } \mathcal{F}=\left\{f_{i}\right\}_{i=1}^{M} \in \mathcal{H}_{N} .
$$

Estos potenciales incluyen el potencial de marco $\left(\operatorname{con} \phi(t)=t^{2}\right)$ y el llamado error cuadrático medio (ECM) (con $\left.\phi(t)=t^{-1}\right)$. En ese mismo trabajo, los autores estudian con esas condiciones de optimalidad un problema de diseño más específico que consiste en completar un conjunto de vectores dados en el contexto finitodimensional. Debido a la necesidad de caracterizar el espectro de los óptimos como el espectro de una suma de matrices autoadjuntas, la desigualdad de Lidskii juega un rol fundamental. Los autores muestran que los minimizadores globales de los potenciales convexos se pueden obtener con completaciones de marcos que satisfacen la igualdad en la desigualdad de Lidskii. Más precisamente, dada una sucesión $\mathcal{F}_{0}=\left\{f_{i}\right\}_{i=1}^{M_{0}}$ de $\mathcal{H}_{N}$ y $\mathcal{G}=\left\{g_{i}\right\}_{M_{0}+1}^{k}$ otra sucesión tal que $\left\{\mathcal{F}_{0}, \mathcal{G}\right\}$ es un marco para $\mathcal{H}_{N}$, los autores probaron el siguiente resultado de minimización:

Teorema 5.1.2. Sea $\mathcal{F}_{0}=\left\{f_{i}\right\}_{i=1}^{M_{0}}$ una sucesión de $\mathcal{H}_{N}$ y $\mathbf{a}=\left(\alpha_{i}\right)_{i=1}^{k}$ una sucesión 
de números positivos tal que $N-\operatorname{rk}\left(S_{\mathcal{F}_{0}}\right) \leq k$. Sea $\lambda$ el vector de autovalores de $S_{\mathcal{F}_{0}}$ y $\phi:[0, \infty) \rightarrow[0, \infty)$ una función estrictamente convexa. Entonces existe un vector $\mu=\mu^{\uparrow}$ con $\mathbf{a} \prec \mu$ tal que

$\left\{\mathcal{F}_{0}, \mathcal{G}\right\}$ es un minimizador de $P_{\phi} \Leftrightarrow \lambda\left(S_{\mathcal{F}}\right)=\left(\lambda\left(S_{\mathcal{F}_{0}}\right)+\lambda^{\uparrow}\left(S_{\mathcal{G}}\right)\right)^{\downarrow}$ y $\lambda^{\uparrow}\left(S_{\mathcal{G}}\right)=\mu$.

\subsubsection{Desigualdad aditiva y minimizadores globales}

En esta sección vemos brevemente cómo las desigualdades de Lidskii se traducen a problemas de minimización de matrices en términos de mayorización. En esencia, esto se debe a que la relación de mayorización entre vectores implica desigualdades de funciones convexas y, por lo tanto, de normas unitariamente invariantes. Nos enfocamos en aquellas matrices que verifican la igualdad que resultan ser minimizadores globales del problema estudiado. Recordamos que los vectores de autovalores, a menos que se especifique lo contrario, se consideran ordenados de forma no creciente.

En [90], los autores estudiaron el caso de igualdad en la desigualdad de Lidskii para dos matrices, de donde se desprende el hecho que sean diagonalizables simultáneamente:

Teorema 5.1.3. Sean $A, B \in \mathcal{H}(d)$. Si $\lambda(A+B)=\left(\lambda^{\uparrow}(A)+\lambda(B)\right)^{\downarrow}$ entonces existe una base ortonormal $\left\{v_{i}\right\}_{i=1}^{d}$ tal que

$$
A=\sum_{i=1}^{d} \lambda_{i}^{\uparrow}(A) v_{i} \otimes v_{i} \quad \text { y } \quad B=\sum_{i=1}^{d} \lambda_{i}(B) v_{i} \otimes v_{i} .
$$

Este resultado nos permite construir, a partir de autovectores de $A$, mínimos para la mayorización como vemos a continuación: dado $\mu \in\left(\mathbb{R}^{d}\right)^{\downarrow}$, si consideramos la órbita unitaria

$$
\mathcal{O}_{\mu}=\left\{G \in \mathcal{M}_{d}(\mathbb{C}): \lambda(G)=\mu\right\}=\left\{V^{*} D_{\mu} V: V \in \mathcal{U}(d)\right\},
$$

por Lidskii se tiene que para todo $G \in \mathcal{O}_{\mu}$,

$$
\lambda(A)^{\uparrow}+\mu \prec \lambda(A+G) .
$$

A partir del Teorema 5.1.3, si existe una matriz $G_{0} \in \mathcal{O}_{\mu}$ tal que

$$
\lambda\left(A+G_{0}\right)=\left(\lambda(A)^{\uparrow}+\lambda\left(G_{0}\right)\right)^{\downarrow}=\lambda(A)^{\uparrow}+\mu,
$$

tenemos entonces que

$$
\lambda\left(A+G_{0}\right) \prec \lambda(A+G) \text { para todo } G \in \mathcal{O}_{\mu} .
$$

Es decir, $G_{0}$ es un mínimo en términos de mayorización sobre las matrices $G \in \mathcal{O}_{\mu}$, por lo que se dice que $G_{0}$ es un minimizador global.

Si $A \in \mathcal{P}(d)$ y $\mu \in\left(\mathbb{R}_{\geq 0}^{d}\right)^{\downarrow}$, notar que las matrices $G$ de la órbita de $\mathcal{O}_{\mu}$ son posi- 
tivas. Para una función estrictamente convexa $\phi: \mathbb{R}_{\geq 0} \rightarrow \mathbb{R}_{\geq 0}$ se define el potencial convexo $\Phi_{\phi}: \mathcal{O}_{\mu} \rightarrow \mathbb{R}_{\geq 0}^{d}$ dado por

$$
\Phi_{\phi}(G)=\operatorname{tr}\left(\phi(A+G)=\sum_{i \in I} \phi\left(\lambda_{i}(A+G)\right) .\right.
$$

Llamamos minimizador global del potencial $\Phi_{\phi}$ a una matriz $G_{1} \in \mathcal{O}_{\mu}$ tal que $\Phi_{\phi}\left(G_{1}\right) \leq \Phi_{\phi}(G)$ para toda $G \in \mathcal{O}_{\mu}$. Usando las propiedades de mayorización para funciones convexas de la Proposición 1.5.3, por (5.2) si $G_{0}$ un minimizador global, $G_{0}$ también resulta un minimizador global de $\Phi_{\phi}$. Recíprocamente, si $G_{1}$ es un minimizador global del potencial $\Phi_{\phi}$ se tiene que

$$
\sum_{i \in I} \phi\left(\lambda_{i}^{\uparrow}(A)+\mu_{i}\right)=\sum_{i \in I} \phi\left(\lambda_{i}\left(A+G_{1}\right)\right) .
$$

Luego, por Lidskii y nuevamente por la Proposición 1.5.3,

$$
\lambda\left(A+G_{1}\right)=\left(\lambda^{\uparrow}(A)+\mu\right)^{\downarrow}=\lambda^{\uparrow}(A)+\lambda\left(G_{1}\right),
$$

es decir, $G_{1}$ es un minimizador global.

Ahora bien, si $A \in \mathcal{H}(d), \mu \in\left(\mathbb{R}^{d}\right)^{\downarrow}$, análogamente a $(5.2)$, sea $G_{0} \in \mathcal{O}_{\mu}$ tal que para todo $G \in \mathcal{O}_{\mu}$,

$$
\left(\lambda(A)^{\uparrow}-\lambda\left(G_{0}\right)\right)^{\downarrow}=\left(\lambda(A)^{\uparrow}-\mu\right)^{\downarrow}=\lambda\left(A-G_{0}\right) \prec \lambda(A-G) .
$$

Si consideramos una norma unitariamente invariante y estrictamente convexa $N$ y definimos la función $\Phi_{N}: \mathcal{O}_{\mu} \rightarrow \mathbb{R}_{\geq 0}^{d}$ dada por

$$
\Phi_{N}(G)=N(A-G),
$$

usando propiedades de mayorización, de (5.4) se tiene que

$$
\Phi_{N}\left(G_{0}\right)=N\left(A-G_{0}\right) \leq N(A-G)=\Phi_{N}(G) .
$$

Esto es, $G_{0}$ es un minimizador global de $\Phi_{N}$. Recíprocamente también, como en el caso del potencial, se puede ver que todo minimizador global de $\Phi_{N}$ verifica la igualdad en (5.4).

En definitiva, caracterizar las matrices que verifican la igualdad de la desigualdad de Lidskii, permite determinar explícitamente los minimizadores globales de las funciones $\Phi_{\phi}$ y $\Phi_{N}$.

\subsubsection{Versión multiplicativa de las desigualdades}

Una de las versiones multiplicativas de las desigualdades de Lidskii se debe a Li y Mathias [84]. En el caso que las matrices sean positivas e inversibles, sus resultados 
pueden relacionarse con el extenso trabajo de desigualdades de valores singulares de Klyachko [78].

Así como sucede para las desigualdades aditivas de Lidskii, caracterizar la igualdad de la versión multiplicativa tiene su correlación con problemas de marcos. Uno de los problemas que los autores estudiaron en [91] es el de perturbación óptima de marcos equivalentes: dado un marco $\mathcal{F}=\left\{f_{j}\right\}_{j \in I_{n}}$ para $\mathbb{C}^{d}$ con $n>d$, el problema consiste en determinar la estructura de un operador inversible $V$ que actúe sobre $\mathbb{C}^{d}$ con el cual se considera el marco perturbado $V(\mathcal{F})=\left\{V f_{j}\right\}_{j \in I_{n}}$. Además, se requiere que $V$ sea próximo a la identidad, una condición de determinante y que la dispersión de los autovalores del operador de marco $S_{\mathcal{V}(\mathcal{F})}$ sea mínima entre todos los autovalores de $S_{\mathcal{V}^{\prime}(\mathcal{F})}$ para todo $\mathcal{V}^{\prime}$. Para este problema, dados $0<\beta<1$ y $s \in\left[(1-\beta)^{d},(1+\beta)^{d}\right]$ se considera el conjunto

$$
\mathcal{O}_{s, \beta}\left(S_{\mathcal{F}}\right)=\left\{V S_{\mathcal{F}} V^{*}: V \in G l(d),\|V-I\| \leq \beta,|\operatorname{det}(V)| \geq s\right\} .
$$

La desigualdad multiplicativa y el caso de igualdad permitieron a los autores mostrar la existencia de elementos óptimos en $\mathcal{O}_{s, \beta}(S)$ y caracterizar su estructura geométrica.

Si consideramos la desigualdad de Gelfand - Naimark - Lidskii (GNL) presentada anteriormente

$$
\log \left(\lambda^{\downarrow}(B)\right)-\log \left(\lambda^{\downarrow}(A)\right) \prec \log \left(\lambda\left(B A^{-1}\right)\right) \prec \log \left(\lambda^{\downarrow}(B)\right)-\log \left(\lambda^{\uparrow}(A)\right)
$$

la caracterización del caso de igualdad de la mayorización (inferior) involucra el siguiente

Teorema 5.1.4 (Teo. 5.1 [91]). Sea $A, B \in \mathcal{P}(d)$ entonces,

$$
(\log (\lambda(B))-\log (\lambda(A)))^{\downarrow}=\log \left(\lambda^{\downarrow}\left(B A^{-1}\right)\right)
$$

si y sólo si existe un unitario $U \in \mathcal{U}(d)$ tal que

$$
U A U^{*}=D_{\lambda(A)} \text { y } U B U^{*}=D_{\lambda(B)} .
$$

Esta caracterización será de utilidad en las pruebas de resultados en el contexto local. Notemos que, nuevamente, los mínimos involucran matrices que conmutan entre sí.

\subsection{Desigualdades de Lidskii locales}

Introducimos en esta sección algunas de las técnicas desarrolladas por Massey, Rios y Stojanoff en [93] para el estudio de la estructura de los minimizadores locales de las funciones $\Phi_{\phi}$ y $\Phi_{N}$. En dicho trabajo se preguntaron si existía alguna versión de los Teoremas de Lidskii (aditivos) que permita caracterizarlos. Producto de estas inquietudes fue que se publicaron una serie de trabajos donde desarrollaron estrategias 
para atacar estos problemas y exhibieron aplicaciones de sus resultados. Estas técnicas nos permiten realizar un estudio local en la igualdad de la desigualdad de Lidskii multiplicativa.

\subsubsection{Desigualdad aditiva local}

Para plantear un escenario local de problema se necesita una estructura de espacio métrico. Notemos que la órbita unitaria $\mathcal{O}_{\mu}$ cuenta con la métrica inducida por la norma espectral. Dadas $A, B \in \mathcal{H}(d)$, se toma $\rho:=\operatorname{tr}(A)+\operatorname{tr}(B)$ y se considera la variedad

$$
\mathcal{H}(d)^{\rho}:=\{C \in \mathcal{H}(d): \operatorname{tr}(C)=\rho\} .
$$

Para la variedad producto $\mathcal{U}(d) \times \mathcal{U}(d)$ se define una aplicación

$$
\Delta: \mathcal{U}(d) \times \mathcal{U}(d) \longrightarrow \mathcal{H}(d)^{\rho}
$$

dada por

$$
\Delta(U, V)=U^{*} A U+V^{*} B V .
$$

En primer lugar, usando argumentos geométricos, se prueba que

$\Delta$ es una submersión en $(I, I)$ si y sólo si $\{A, B\}^{\prime}=\mathbb{C} \cdot I$.

Intuitivamente, contar con una submersión en $(I, I)$ nos permite 'traer' condiciones locales en $A+B \in \mathcal{H}^{\rho}$ a condiciones locales en un entorno de $(I, I) \in \mathcal{U}(d) \times \mathcal{U}(d)$. Esto resulta de utilidad para los siguientes resultados, algunos de los cuales adaptamos a la versión multiplicativa local.

La idea inicial es mostrar que, si una matriz no verifica la igualdad de Lidskii o no conmuta con $A$, no puede ser un mínimo. Respecto a este último caso, notar la exigencia de la condición de conmutante $\{A, B\}^{\prime}$ trivial mencionada en líneas anteriores. En cualquiera de estos casos, la estrategia es construir una curva suave $\gamma(t)$ en la variedad tal que el espectro de $\gamma(t)$ esté mayorizado por $A+B$.

Proposición 5.2.1. Sean $A, B \in \mathcal{H}(d)$ tales que $[A, B] \neq 0$. Entonces, dado $\varepsilon>0$ existe $W \in \mathcal{U}(d)$ tal que $\|I-W\|<\varepsilon y$

$$
\lambda\left(A+W^{*} B W\right) \prec \lambda(A+B) \text { pero } \lambda\left(A+W^{*} G W\right) \neq \lambda(A+B) .
$$

El siguiente teorema muestra que para tener un mínimo $G_{0}$ se debe verificar la igualdad de la desigualdad de Lidskii.

Teorema 5.2.2. Sean $A \in \mathcal{P}(d)$ y $\mu \in\left(\mathbb{R}^{d}\right)^{\downarrow}$. Sea $G_{0} \in \mathcal{O}_{\mu}$ tal que

$$
\lambda\left(A+G_{0}\right) \neq\left(\lambda(A)^{\uparrow}+\lambda\left(G_{0}\right)\right)^{\downarrow}
$$


Luego, dado $\varepsilon>0$, existe $G \in \mathcal{O}_{\mu}$ tal que $\left\|G-G_{0}\right\|<\varepsilon y$

$$
\left.\lambda(A+G) \prec \lambda\left(A+G_{0}\right)\right) \text { pero } \lambda(A+G) \neq \lambda\left(A+G_{0}\right) .
$$

Con los resultados anteriores se probaron las siguientes versiones locales para los problemas de minimización de potenciales convexos y NUIs, respectivamente:

Teorema 5.2.3. Sea $A \in \mathcal{P}(d), \mu=\left(\mu_{i}\right)_{i \in \mathbb{I}_{d}} \in\left(\mathbb{R}_{\geq 0}^{d}\right)^{\downarrow}$ y $\phi$ una función estrictamente convexa. Si $G_{0}$ es un minimizador local de $\Phi_{\phi}$ en $\mathcal{O}_{\mu}$, existe una base ortonormal $\left\{v_{i}\right\}_{i \in \mathbb{I}_{d}}$ tal que

$$
A=\sum_{i=1}^{d} \lambda_{i}^{\uparrow}(A) v_{i} \otimes v_{i} \quad \text { y } \quad G_{0}=\sum_{i=1}^{d} \mu_{i} v_{i} \otimes v_{i} .
$$

En particular, $\lambda\left(A+G_{0}\right)=\left(\lambda(A)^{\uparrow}+\lambda\left(G_{0}\right)\right)^{\downarrow}$, por lo que $G_{0}$ es también un minimizador global de $\Phi_{\phi}$.

Teorema 5.2.4. Sea $A \in \mathcal{P}(d), \mu=\left(\mu_{i}\right)_{i \in \mathbb{I}_{d}} \in\left(\mathbb{R}_{\geq 0}^{d}\right)^{\downarrow}$ y N una NUI estrictamente convexa en $\mathcal{M}_{d}$. Si $G_{0}$ es un minimizador local de $\Phi_{N}$ en $\mathcal{O}_{\mu}$, existe una base ortonormal $\left\{v_{i}\right\}_{i \in \mathbb{I}_{d}}$ tal que

$$
A=\sum_{i=1}^{d} \lambda_{i}^{\uparrow}(A) v_{i} \otimes v_{i} \quad \text { y } \quad G_{0}=\sum_{i=1}^{d} \mu_{i} v_{i} \otimes v_{i} .
$$

En particular, $\lambda\left(A-G_{0}\right)=\left(\lambda(A)-\lambda\left(G_{0}\right)\right)^{\downarrow}$, por lo que $G_{0}$ es también un minimizador global de $\Phi_{N}$ en $\mathcal{O}_{\mu}$.

\subsubsection{Desigualdad multiplicativa local}

En esta sección presentamos los resultados que utilizaremos para el estudio local en la igualdad de la mayorización inferior de la desigualdad de Lidskii multiplicativa que nos servirá para caracterizar los mínimos locales en el problema de Procrusto. A grandes rasgos, el esquema de demostración tiene la misma estructura que el caso aditivo de la sección anterior. Un estudio similar se puede hacer para la igualdad superior en dicha mayorización obteniendo un máximo local.

Consideramos

$$
\mathcal{P}(d)^{\tau}:=\{C \in \mathcal{P}(d): \operatorname{det}(C)=\tau\}
$$

siendo $\tau=\frac{\operatorname{det}(B)}{\operatorname{det}(A)}$. En la variedad producto $\mathcal{U}(d) \times \mathcal{U}(d)$ definimos una aplicación

$$
\Gamma: \mathcal{U}(d) \times \mathcal{U}(d) \longrightarrow \mathcal{P}(d)^{\tau} \text { dada por } \Gamma(U, V)=U A^{-1 / 2} U^{*} V B V^{*} U A^{-1 / 2} U^{*}
$$

Como en el caso aditivo, en el siguiente lema, probamos que $\Gamma$ es una submersión en $(I, I)$ cuando se tiene que $\{A, B\}^{\prime}=\mathbb{C} \cdot I$.

Lema 5.2.5. $\Gamma$ es una submersión en $(I, I) \in \mathcal{U}(d) \times \mathcal{U}(d)$ si y sólo si

$$
\{A, B\}^{\prime}:=\left\{C \in \mathcal{M}_{d}(\mathbb{C}): A C=C A, B C=C B\right\}=\mathbb{C} \cdot I .
$$


Demostración. Supongamos que el conmutante relativo $\{A, B\}^{\prime}$ es el trivial. Para probar que $\Gamma$ es una submersión en $(I, I)$ necesitamos mostrar que la aplicación diferencial

$$
D_{(I, I)} \Gamma: \mathcal{T}_{(I, I)} \mathcal{U}(d) \times \mathcal{U}(d) \longrightarrow \mathcal{T}_{A^{-1 / 2} B A^{-1 / 2}} \mathcal{P}(d)^{\tau}
$$

es suryectiva.

Supongamos que $D_{(I, I)} \Gamma$ no es suryectiva. Debido a la estructura riemanniana del espacio tangente en $\mathcal{P}(d)^{\tau}$, existe $Y_{0} \in \mathcal{T}_{A^{-1 / 2} B A^{-1 / 2}} \mathcal{P}(d)^{\tau}, Y_{0} \neq 0$ tal que

$$
\left\langle D_{(I, I)} \Gamma\left(X_{1}, X_{2}\right), Y_{0}\right\rangle=\operatorname{tr}\left(D_{(I, I)} \Gamma\left(X_{1}, X_{2}\right) Y_{0}^{*}\right)=0,
$$

para cada $\left(X_{1}, X_{2}\right) \in \mathcal{T}_{(I, I)} \mathcal{U}(d) \times \mathcal{U}(d)$. Como

$$
\mathcal{T}_{(I, I)} \mathcal{U}(d) \times \mathcal{U}(d)=\left\{\left(X_{1}, X_{2}\right) \in \mathcal{M}_{d}(\mathbb{C}) \times \mathcal{M}_{d}(\mathbb{C}): X_{i}^{*}=-X_{i}, i=1,2\right\}
$$

tomando una curva en $\mathcal{P}(d)^{\tau}$ es fácil de ver que

$$
\mathcal{T}_{A^{-1 / 2} B A^{-1 / 2}} \mathcal{P}(d)^{\tau}=\left\{Y \in \mathcal{H}(d): \operatorname{tr}\left(\left(A^{1 / 2} B^{-1} A^{1 / 2}\right) Y\right)=0\right\}
$$

por lo que $Y_{0} \in \mathcal{H}(d)$ y verifica que $\operatorname{tr}\left(C Y_{0} C\right)=0$, siendo $C=\left(A^{-1 / 2} B A^{-1 / 2}\right)^{-1 / 2}$. Luego, considerando las derivadas parciales

$$
D_{(I, I)} \Gamma(0, X)=A^{-1 / 2}[X, B] A^{-1 / 2}
$$

$\mathrm{y}$

$$
D_{(I, I)} \Gamma(X, 0)=\left[X, A^{-1 / 2}\right] B A^{-1 / 2}+A^{-1 / 2} B\left[X, A^{-1 / 2}\right],
$$

por (5.7) se tiene que

$$
\operatorname{tr}\left(A^{-1 / 2}[X, B] A^{-1 / 2} Y_{0}\right)=\operatorname{tr}\left(\left[B, A^{-1 / 2} Y_{0} A^{-1 / 2}\right] X\right)=0
$$

para todo $X^{*}=-X$. En particular, para $X=\left[B, A^{-1 / 2} Y_{0} A^{-1 / 2}\right]$, se deduce que

$$
\left[B, A^{-1 / 2} Y_{0} A^{-1 / 2}\right]=0
$$

Nuevamente, por (5.7) y (5.9),

$$
\begin{aligned}
& \operatorname{tr}\left(\left[X, A^{-1 / 2}\right] B A^{-1 / 2} Y_{0}\right)+\operatorname{tr}\left(A^{-1 / 2} B\left[X, A^{-1 / 2}\right] Y_{0}\right)= \\
& =\operatorname{tr}\left(\left(\left[A^{-1 / 2}, B A^{-1 / 2} Y_{0}\right]+\left[A^{-1 / 2}, Y_{0} A^{-1 / 2} B\right]\right) X\right)=0
\end{aligned}
$$

para todo $X^{*}=-X$. Entonces, se tiene que

$$
\left[A^{-1 / 2}, B A^{-1 / 2} Y_{0}\right]+\left[A^{-1 / 2}, Y_{0} A^{-1 / 2} B\right]=0
$$


De $(5.10)$ y $(5.11)$ obtenemos que $\left[Y_{0}, A^{-1 / 2} B A^{-1 / 2}\right]=0$, es decir,

$$
B A^{-1 / 2} Y_{0} A^{-1 / 2} A=A A^{-1 / 2} Y_{0} A^{-1 / 2} B
$$

Finalmente, como $\left[B^{1 / 2}, A^{-1 / 2} Y_{0} A^{-1 / 2}\right]=0$, deducimos que

$$
B^{1 / 2} A^{-1 / 2} Y_{0} A^{-1 / 2} B^{1 / 2} \in\{A, B\}^{\prime} .
$$

Entonces, por hipótesis, $B^{1 / 2} A^{-1 / 2} Y_{0} A^{-1 / 2} B^{1 / 2}=\alpha \cdot I$, para algún escalar $\alpha$. Esto es,

$$
Y_{0}=\alpha\left(A^{1 / 2} B^{-1} A^{1 / 2}\right)
$$

Luego, $0=\operatorname{tr}\left(A^{1 / 2} B^{-1} A^{1 / 2} Y_{0}\right)=\alpha \cdot \operatorname{tr}\left(\left(A^{1 / 2} B^{-1} A^{1 / 2}\right)^{2}\right)$ lo que contradice que $Y_{0} \neq 0$. Por lo tanto, $\Gamma$ es una submersión en $(I, I)$.

Recíprocamente, supongamos que existe una proyección ortogonal no trivial $P$ en $\{A, B\}^{\prime}$. Sea $Z \in \mathcal{H}(d) \cap\{A, B\}^{\prime}$ dado por

$$
Z:=P+\frac{c}{c-\operatorname{tr}\left(\left(A^{1 / 2} B^{-1} A^{1 / 2}\right)^{2}\right)}(I-P),
$$

con $c=\operatorname{tr}\left(P\left(A^{1 / 2} B^{-1} A^{1 / 2}\right)^{2}\right)$. Notar que, mediante cálculo funcional, $P$ conmuta con $A^{1 / 2} B^{-1} A^{1 / 2}$. Entonces, si consideramos

$$
Y_{0}:=A^{1 / 2} B^{-1 / 2} Z B^{-1 / 2} A^{1 / 2}=A^{1 / 2} B^{-1} A^{1 / 2} Z,
$$

$Y_{0} \in \mathcal{H}(d)$, es no trivial y verifica que

$$
\begin{aligned}
\operatorname{tr}\left(A^{1 / 2} B^{-1} A^{1 / 2} Y_{0}\right) & =\operatorname{tr}\left(\left(A^{1 / 2} B^{-1} A^{1 / 2}\right)^{2} Z\right) \\
& =\operatorname{tr}\left(P\left(A^{1 / 2} B^{-1} A^{1 / 2}\right)^{2}\right)+ \\
& +\frac{c}{c-\operatorname{tr}\left(\left(A^{1 / 2} B^{-1} A^{1 / 2}\right)^{2}\right)} \operatorname{tr}\left((I-P)\left(A^{1 / 2} B^{-1} A^{1 / 2}\right)^{2}\right) \\
& =c+c\left(\frac{\operatorname{tr}\left(\left(A^{1 / 2} B^{-1} A^{1 / 2}\right)^{2}\right)}{c-\operatorname{tr}\left(\left(A^{1 / 2} B^{-1} A^{1 / 2}\right)^{2}\right)}-\frac{c}{c-\operatorname{tr}\left(\left(A^{1 / 2} B^{-1} A^{1 / 2}\right)^{2}\right)}\right) \\
& =0 .
\end{aligned}
$$

Esto es, $Y_{0} \in \mathcal{T}_{A^{-1 / 2} B A^{-1 / 2}} \mathcal{P}(d)^{\tau}$. Es fácil ver que

$$
\left[A^{-1 / 2} Y_{0} A^{-1 / 2}, B\right]=\left[B A^{-1 / 2} Y_{0}, A^{-1 / 2}\right]=\left[Y_{0} A^{-1 / 2} B, A^{-1 / 2}\right]=0,
$$

lo que implica que $Y_{0}$ es ortogonal al rango de $D_{(I, I)} \Gamma$, por (5.8) y (5.9), $Y_{0}$ es ortogonal al rango de $D_{(I, I)} \Gamma$, por lo tanto $\Gamma$ no es una submersión en $(I, I)$.

El lema anterior nos permite considerar el caso donde $A$ y $B$ no conmutan. Recordar que el objetivo es caracterizar a los mínimos locales a partir de la condición de igualdad en la mayorización. Esto nos resultará de utilidad para probar el Teorema 
5.2 .7 .

Lema 5.2.6. Sean $A, B \in \mathcal{P}(d)$ tales que $[A, B] \neq 0$. Entonces, para $t_{0}>0$ suficientemente pequeño, existe una curva continua

$$
\gamma(t):\left(-t_{0}, t_{0}\right) \longrightarrow \mathcal{U}(d) \text { tal que } \gamma(0)=I
$$

$y$

$$
\log \lambda\left(A^{-1} B\right) \neq \log \lambda\left(A^{-1} \gamma(t) B \gamma(t)^{*}\right) \prec \log \lambda\left(A^{-1} B\right) \quad \text { para } \quad t \neq 0 .
$$

Demostración. Como $[A, B] \neq 0$, existe una proyección ortogonal $P$ de la ${ }^{*}$-subálgebra unital $\{A, B\}^{\prime} \subseteq \mathcal{M}_{d}(\mathbb{C})$ tal que $[P A, P B] \neq 0$. Esto implica que el rango de $P$ es estrictamente mayor a 1 (de lo contrario, $[P A, P B]=0$ ). Podemos asumir que $P$ es una proyección minimal con esta propiedad.

Claramente, $I \in\{A, B\}^{\prime}$ es una proyección que satisface $[I A, I B] \neq 0$. Si $I$ no es una proyección minimal en $\{A, B\}^{\prime}$ entonces existen $P_{1}, P_{2} \in\{A, B\}^{\prime}$ proyecciones no nulas tales que $I=P_{1}+P_{2}$ y $\left[P_{i} A, P_{i} B\right] \neq 0$ con $i=1,2$. Si ninguna de las proyecciones $P_{i}$ es minimal en $\{A, B\}^{\prime}$ podemos repetir el argumento anterior a $P_{i}$ y, como estamos trabajando con álgebras de dimensión finita, del procedimiento obtenemos una proyección minimal $P \in\{A, B\}^{\prime}$ con la propiedad mencionada al principio de la prueba. Finalmente, aplicando un cambio de base ortonormal, podemos asumir que $\operatorname{Im}(P)=\operatorname{span}\left\{e_{i}: i \in \mathbb{I}_{r}\right\}$, con $r=\operatorname{rk}(P)>1$. Como $P$ reduce $A$ y $B$ podemos considerar

$$
A_{1}=\left.A\right|_{\operatorname{Im}(P)} \in \mathcal{P}(r) \text { y } B_{1}=\left.B\right|_{\operatorname{Im}(P)} \in \mathcal{P}(r) .
$$

Entonces, tenemos que $\left\{A_{1}, B_{1}\right\}^{\prime}=\mathbb{C} \cdot I_{r} \subset \mathcal{M}_{r}(\mathbb{C})\left(I_{r}\right.$ es la matriz identidad en $\mathcal{M}_{r}(\mathbb{C})$ ); de hecho, si existiera una proyección no trivial $Q$ en $\left\{A_{1}, B_{1}\right\}^{\prime}$ tal que $\left[Q A_{1}, Q B_{1}\right] \neq 0$, la podemos dilatar a una proyección $\bar{Q}$ en $\{A, B\}^{\prime}$ tal que $\bar{Q}<P$ y $[\bar{Q} A, \bar{Q} B] \neq 0$. Pero esto contradeciría la minimalidad de $P$.

Sea $\sigma=\frac{\operatorname{det}\left(B_{1}\right)}{\operatorname{det}\left(A_{1}\right)}$. Consideremos $\Gamma: \mathcal{U}(r) \times \mathcal{U}(r) \longrightarrow \mathcal{P}(r)^{\sigma}$ definida como

$$
\Gamma(U, V)=U A_{1}^{-1 / 2} U^{*} V B_{1} V^{*} U A_{1}^{-1 / 2} U^{*}
$$

Por Lemma 5.2.5, $\Gamma$ es una submersión en $\left(I_{r}, I_{r}\right)$ sobre $\mathcal{P}(r)^{\sigma}$. En particular, esto implica que toda curva continua $l(t):(-1,1) \longrightarrow \mathcal{P}(r)^{\sigma}$ tal que $l(0)=A_{1}^{-1 / 2} B_{1} A_{1}^{-1 / 2}$, puede ser levantada (localmente) a una curva continua

$$
\widetilde{l}(t):\left(-t_{0}, t_{0}\right) \longrightarrow \mathcal{U}(r) \times \mathcal{U}(r)
$$

tal que $\widetilde{l}(0)=\left(I_{r}, I_{r}\right)$ y $l(t)=\Gamma \circ \widetilde{l}(t)$, para $-t_{0}<t<t_{0}$.

Como $\left[A_{1}, B_{1}\right] \neq 0$, del Teorema 5.1.4 podemos asegurar que

$$
b:=\log \lambda\left(A_{1}^{-1 / 2} B_{1} A_{1}^{-1 / 2}\right) \neq\left(\log \lambda\left(B_{1}\right)-\log \lambda\left(A_{1}\right)\right)^{\downarrow}:=a .
$$

Más aún, por el Teorema Gelfand-Naimark-Lidskii, ocurre que $a \prec b$. Asimismo, $\rho(t):=|t| a+(1-|t|) b \prec b$, para $t \in(-1,1)$, con $\rho(t) \neq b$ para $t \neq 0$. Sea $U \in \mathcal{U}(r)$ 
tal que

$$
U D_{\lambda\left(A_{1}^{-1 / 2} B_{1} A_{1}^{-1 / 2}\right)} U^{*}=A_{1}^{-1 / 2} B_{1} A_{1}^{-1 / 2}
$$

donde $D_{\lambda\left(A_{1}^{-1 / 2} B_{1} A_{1}^{-1 / 2}\right)}$ denota la matriz diagonal con los autovalores de $A_{1}^{-1 / 2} B_{1} A_{1}^{-1 / 2}$ dispuestos en orden no creciente; y sea $l(t):(-1,1) \longrightarrow \mathcal{P}(r)^{\sigma}$ dada por

$$
l(t)=U D_{\exp (\rho(t))} U^{*},
$$

donde $\exp (\rho(t)):=\left(e^{\rho(t)_{1}}, e^{\rho(t)_{2}}, \ldots, e^{\rho(t)_{r}}\right) \in \mathbb{R}^{r}$. Tenemos entonces que $l(t)$ es una curva continua tal que $l(0)=A_{1}^{-1 / 2} B_{1} A_{1}^{-1 / 2}$. Más aún,

$$
\operatorname{det}(l(t))=\Pi_{i=1}^{r} e^{\rho(t)_{i}}=e^{\operatorname{tr} \rho(t)}=\frac{\operatorname{det}\left(B_{1}\right)}{\operatorname{det}\left(A_{1}\right)}=\sigma
$$

por lo que $l(t) \in \mathcal{P}(r)^{\sigma}, \forall t \in(-1,1)$. Además, por construcción, $\log \lambda(l(t)) \prec$ $\log \lambda(l(0))$, para $t \in(-1,1)$.

Luego, para $0<t_{0}$ suficientemente pequeño, existe una curva continua $\widetilde{l}(t)=$ $(U(t), V(t)) \in \mathcal{U}(r) \times \mathcal{U}(r), t \in\left(-t_{0}, t_{0}\right)$ tal que $\widetilde{l}(0)=\left(I_{r}, I_{r}\right)$ y $\Gamma \circ \widetilde{l}(t)=l(t)$. Consideramos entonces

$$
\widetilde{\gamma}(t):\left(-t_{0}, t_{0}\right) \longrightarrow \mathcal{U}(r) \text { dada por } \widetilde{\gamma}(t)=U^{*}(t) V(t)
$$

$\widetilde{\gamma}(t)$ es continua, $\widetilde{\gamma}(0)=I_{r} \mathrm{y}$

$$
\begin{aligned}
\log \lambda\left(A_{1}^{-1} \widetilde{\gamma}(t) B_{1} \widetilde{\gamma}^{*}(t)\right) & =\log \lambda\left(A_{1}^{-1} V^{*}(t) U(t) B_{1} U^{*}(t) V(t)\right) \\
& =\log \lambda\left(U(t) A_{1}^{-1 / 2} U^{*}(t) V(t) B_{1} V^{*}(t) U(t) A_{1}^{-1 / 2} U^{*}(t)\right) \\
& =\log \lambda(l(t)) \prec \log \lambda\left(A_{1}^{-1} B_{1}\right) .
\end{aligned}
$$

Finalmente, dilatamos $\widetilde{\gamma}(t):\left(-t_{0}, t_{0}\right) \longrightarrow \mathcal{U}(r)$ a una curva $\gamma(t):\left(-t_{0}, t_{0}\right) \longrightarrow \mathcal{U}(d)$ tal que

$$
\gamma(t)=\left(\begin{array}{cc}
\widetilde{\gamma}(t) & 0 \\
0 & I_{d-r}
\end{array}\right)
$$

donde la partición por bloques se considera respecto a $\mathbb{C}^{d}=\operatorname{Im}(P) \oplus \operatorname{Im}(P)^{\perp}$ con un cambio de base apropiado. Como $P \in\{A, B\}^{\prime}$, respecto a los bloques tenemos que

$$
A=\left(\begin{array}{cc}
A_{1} & 0 \\
0 & A_{2}
\end{array}\right) \quad \text { y } B=\left(\begin{array}{cc}
B_{1} & 0 \\
0 & B_{2}
\end{array}\right)
$$

con lo cual

$$
A^{-1} \gamma(t) B \gamma(t)^{*}=\left(\begin{array}{cc}
A_{1}^{-1} \widetilde{\gamma}(t) B_{1} \widetilde{\gamma}(t)^{*} & 0 \\
0 & A_{2}^{-1} B_{2} .
\end{array}\right)
$$

Por lo tanto, como $\lambda\left(A^{-1} B\right)=\left(\lambda\left(A_{1}^{-1} B_{1}\right), \lambda\left(A_{2}^{-1} B_{2}\right)\right)^{\downarrow} \in\left(\mathbb{R}^{d}\right)^{\downarrow}$ y los autovalores 
(ordenados) de $A^{-1} \gamma(t) B \gamma(t)^{*}$ son

$$
\left(\lambda\left(A_{1}^{-1} \widetilde{\gamma}(t) B_{1} \widetilde{\gamma}(t)^{*}\right), \lambda\left(A_{2}^{-1} B_{2}\right)\right)^{\downarrow} \in\left(\mathbb{R}^{d}\right)^{\downarrow},
$$

es fácil probar que $\gamma(t)$ tiene las propiedades requeridas.

En concreto, vimos que si $A$ y $B$ no conmutan, se puede construir una curva de matrices $\gamma(t) B \gamma(t)^{*}$ (de la órbita de $B$ ) que está mayorizada por $A^{-1} B$. Por lo tanto, $B$ no sería un mínimo local.

Teorema 5.2.7. Sean $A, B \in \mathcal{P}(d)$ tales que

$$
\log \lambda\left(A^{-1} B\right) \neq(\log \lambda(B)-\log \lambda(A))^{\downarrow}
$$

Entonces, para algún $t_{0}>0$ suficientemente pequeño, existe una curva continua

$$
\gamma(t):\left(-t_{0}, t_{0}\right) \longrightarrow \mathcal{U}(d) \text { tal que } \gamma(0)=I \text { y } y
$$

$$
\log \lambda\left(A^{-1} B\right) \neq \log \lambda\left(A^{-1} \gamma(t) B \gamma(t)^{*}\right) \prec \log \lambda\left(A^{-1} B\right) \text { para } t \neq 0 .
$$

Demostración. Por Lema 5.2.6 alcanza con probar el caso $[A, B]=0$, por lo que podemos suponer que $A B=B A$. Si denotamos a $\lambda^{\sigma}(B)$ por los autovalores de $B$ sin orden específico, sea $U \in \mathcal{U}(d)$ tal que

$$
U A^{-1} B U^{*}=D_{\lambda\left(A^{-1}\right)} D_{\lambda^{\sigma}(B)},
$$

donde $D_{\lambda_{\left(A^{-1}\right)}}$ y $D_{\lambda^{\sigma}(B)}$ son matrices diagonales con

$$
\lambda\left(A^{-1}\right)=\left(\lambda(A)_{1}^{-1}, \lambda(A)_{2}^{-1}, \ldots, \lambda(A)_{d}^{-1}\right)
$$

arreglados en orden no decreciente. Supongamos que para algún $1 \leq i \leq d$, se tiene que $\lambda_{i}^{\sigma}(B)<\lambda_{i+1}^{\sigma}(B)$. Sea $\left\{e_{j}\right\}_{j=1}^{d}$ una base ortonormal de $A$ y $B$ asociada a $U$.

Por simplicidad denotamos $\alpha_{1}=\lambda(A)_{i}, \alpha_{2}=\lambda(A)_{i+1}$ y $\beta_{1}=\lambda_{i}^{\sigma}(B), \beta_{2}=\lambda_{i+1}^{\sigma}(B)$. Entonces, si nos restringimos al subespacio $\mathcal{S}$ generado por los autovectores $\left\{e_{i}, e_{i+1}\right\}$,

$$
U A^{-1} B U^{*} \uparrow_{\mathcal{S}}=\left[\begin{array}{cc}
\frac{\beta_{1}}{\alpha_{1}} & 0 \\
0 & \frac{\beta_{2}}{\alpha_{2}}
\end{array}\right] \text {. }
$$

Sea $W(t) \in \mathcal{U}(d)$, para $t \in\left(-\frac{\pi}{2}, \frac{\pi}{2}\right)$, dado por

$$
W(t)=\cos (t)\left(e_{i} \otimes e_{i}+e_{i+1} \otimes e_{i+1}\right)+\sin (t)\left(e_{i} \otimes e_{i+1}-e_{i+1} \otimes e_{i}\right)+P_{\mathcal{S}^{\perp}}
$$

Entonces, consideremos $C_{\mathcal{S}}(t):=U A^{-1} U^{*} W(t) U B U^{*} W^{*}(t) \uparrow_{\mathcal{S}}$. Notar que $C_{\mathcal{S}}(t)$ verifica que

$$
C_{\mathcal{S}}(t)=\left[\begin{array}{cc}
\alpha_{1}^{-1} & 0 \\
0 & \alpha_{2}^{-1}
\end{array}\right]\left[\begin{array}{cc}
\cos (t) & \sin (t) \\
-\sin (t) & \cos (t)
\end{array}\right]\left[\begin{array}{cc}
\beta_{1} & 0 \\
0 & \beta_{2}
\end{array}\right]\left[\begin{array}{cc}
\cos (t) & -\sin (t) \\
\sin (t) & \cos (t)
\end{array}\right]
$$


Es fácil de ver que para $t \neq 0$,

$$
\operatorname{tr}\left(C_{\mathcal{S}}(t)\right)=\frac{\beta_{1}}{\alpha_{1}}+\frac{\beta_{2}}{\alpha_{2}}+\sin ^{2}(t)\left(\beta_{1}-\beta_{2}\right)\left(\alpha_{2}^{-1}+\alpha_{1}^{-1}\right)<\frac{\beta_{1}}{\alpha_{1}}+\frac{\beta_{2}}{\alpha_{2}}=\operatorname{tr}\left(C_{\mathcal{S}}(0)\right) .
$$

Como $\operatorname{det}\left(C_{\mathcal{S}}(t)\right)=\frac{\beta_{1} \beta_{2}}{\alpha_{1} \alpha_{2}}$ para todo $t$, esto implica que los autovalores $\lambda_{1}\left(C_{\mathcal{S}}\right) \geq$ $\lambda_{2}\left(C_{\mathcal{S}}\right)$ satisfacen

$$
\left(\log \left(\lambda_{1}\left(C_{\mathcal{S}}(t)\right)\right), \log \left(\lambda_{2}\left(C_{\mathcal{S}}(t)\right)\right)\right) \prec\left(\log \left(\frac{\beta_{1}}{\alpha_{1}}\right), \log \left(\frac{\beta_{2}}{\alpha_{2}}\right)\right)
$$

Finalmente, si tomamos $\gamma(t):=U^{*} W(t) U$ con $-\frac{\pi}{2} \leq t \leq \frac{\pi}{2}$, es claro que para todo $t$,

$$
\log \lambda\left(A^{-1} \gamma(t) B \gamma^{*}(t)\right)=\log \left(\lambda\left(U A^{-1} U^{*} W(t) U B U^{*} W^{*}(t)\right) \prec \log \lambda\left(A^{-1} B\right),\right.
$$

ya que $A^{-1} B P_{\mathcal{S}^{\perp}}=A^{-1} \gamma(t) B \gamma^{*}(t) P_{\mathcal{S}^{\perp}}$.

\subsection{Caracterización de extremos locales en un pro- blema de Procusto}

En esta sección aplicaremos los resultados de la sección anterior para caracterizar los mínimos locales de un problema de Procusto estudiado por Bhatia y Congedo. Más precisamente, mostramos que los minimizadores globales para el problema de Procusto, en el caso particular de la familia de distancias log, sólo pueden ser aquellos puntos propuestos en ese trabajo [9]. Como en las secciones anteriores, la sección comienza con una motivación breve del estudio del problema de Procusto.

\subsubsection{Motivación: caso ortogonal}

Una de las aplicaciones de los problemas de Procusto que podemos mencionar brevemente es el caso del problema ortogonal. Supongamos que, a partir de ciertos experimentos, se obtienen datos en forma de una matriz $A \in \mathcal{M}_{n}(\mathbb{R})$ y, luego de repetirlos, se obtiene otra matriz distinta $B \in \mathcal{M}_{n}(\mathbb{R})$. Para evaluar qué tan cerca está $B$ de ser una rotación de la matriz $A$ se plantea el siguiente problema:

$$
\min _{X^{t} X=I}\|A-B X\|_{F}
$$

siendo $\|.\|_{F}$ la norma Frobenius. Por la ortogonalidad de $X$, se tiene que

$$
\|A-B X\|_{F}^{2}=\|A\|_{F}^{2}+\|B X\|_{F}^{2}-2 \sum_{k=1}^{p}\left[X^{t}\left(B^{t} A\right)\right]_{k k}=\|A\|_{F}^{2}+\|B\|_{F}^{2}-2 \operatorname{tr}\left(X^{t}\left(B^{t} A\right)\right) .
$$


Con lo cual, resolver el problema (5.12) es equivalente a calcular

$$
\operatorname{máx}_{X^{t} X=I} \operatorname{tr}\left(X^{t} B^{t} A\right) \text {. }
$$

Si se considera la descomposición en valores singulares

$$
B^{t} A=U D_{S\left(B^{t} A\right)} V^{t}
$$

siendo $s_{1}, \ldots, s_{n}$ los valores singulares de $B^{t} A$, y se toma $Z:=V^{t} X^{t} U$ se tiene que

$$
\operatorname{tr}\left(X^{t} B^{t} A\right)=\operatorname{tr}\left(X^{t} U D_{S\left(B^{t} A\right)} V^{t}\right)=\operatorname{tr}\left(Z D_{S\left(B^{t} A\right)}\right)=\sum_{i=1}^{n} z_{i i} s_{i} \leq \sum_{i=1}^{n} s_{i}
$$

donde $z_{i i} \leq 1$ para $i$ ya que $Z$ es producto de ortogonales. Luego, la cota superior se puede alcanzar tomando $Z=I$, es decir, $X=U V^{t}$. Naturalmente, este tipo de estrategias para enfrentar el problema de Procusto induce algoritmos que resultan de gran utilidad en el campo aplicado. Para profundizar en el enfoque algorítmico sugerimos [64].

\subsubsection{Distancias log}

En el problema doble de Procusto, Bhatia y Congedo consideraron distintas distancias para su estudio. Los autores consideraron la distancia Riemmaniana

$$
d_{2}(A, B)=\left\|\log \left(A^{-1 / 2} B A^{1 / 2}\right)\right\|_{2}=\left[\sum_{k=1}^{d} \log ^{2} \lambda_{k}\left(A^{-1} B\right)\right]^{1 / 2}
$$

y el objetivo es buscar el

$$
\min _{U \in \mathcal{O}_{A}} d_{2}\left(A, U^{*} B U\right)=\min _{U \in \mathcal{O}_{A}} d_{2}(A, B)=\left\|\log \left(A^{-1 / 2} B A^{1 / 2}\right)\right\|_{2}
$$

siendo $\|A\|_{2}=\left(\operatorname{tr} A^{*} A\right)^{1 / 2}$, tomando $U$ en la órbita unitaria de $A$.

Ellos notaron que la métrica de este problema se encuentra dentro de las funciones gauge simétricas. Recordamos que esta clase de funciones guardan una estrecha relación con las normas unitariamente invariante como vimos en los preliminares. Las métricas inducidas en $\mathcal{P}(d)$ por cada función gauge $\Phi$ en $\mathbb{R}^{d}$ están dadas por

$$
d_{\Phi}(A, B)=\left\|\log \left(A^{-1 / 2} B A^{1 / 2}\right)\right\|_{\Phi}=\Phi\left(\log \lambda\left(A^{-1} B\right)\right) .
$$

La distancia $d_{2}$ corresponde con el caso donde $\Phi$ representa la norma euclídea.

En las aplicaciones son diversas las distancias que resultan de utilidad que tienen este origen en común. La distancia $d_{2}$ se puede considerar como la versión matricial de la llamada métrica de Fisher-Rao en distribución de probabilidades; la distancia 
Bures-Wasserstein

$$
d_{W}(A, B)=\left[\operatorname{tr}(A+B)-2 \operatorname{tr}\left(A^{1 / 2} B A^{1 / 2}\right)^{1 / 2}\right]^{1 / 2} .
$$

aparece en teoría de transporte óptimo, información cuántica y geometría Riemanniana. Otras distancias de gran utilidad son las conocidas como divergencia de KullbackLeibler, dada por

$$
d_{K L}^{2}(A, B)=\operatorname{tr}\left(A B^{-1}-I\right)-\log \operatorname{det}\left(A B^{-1}\right),
$$

y la divergencia de Bhattacharyya:

$$
d_{B}^{2}(A, B)=\log \operatorname{det}\left(\frac{A+B}{2}\right)-\log \operatorname{det}\left(A^{1 / 2} B^{1 / 2}\right) .
$$

Englobando a estas distancias con la definida en (5.13) el resultado que nos interesa es el siguiente:

Teorema 5.3.1. (Bhatia, Congedo) Sean $A, B$ definidas positivas en $\mathcal{M}_{d}(\mathbb{C})$ y $N$ una NUI. Sean $U, W \in \mathcal{U}(d)$ tales que

$$
W^{*} B W=D_{\lambda(B)} \quad \text { y } \quad V^{*} A V=D_{\lambda(A)}
$$

entonces (5.14) alcanza su minimo en $W V^{*}$.

\subsubsection{Caracterización de los mínimos locales}

Dados $A, B \in \mathcal{P}(d)$ y $N(\cdot)$ una norma unitariamente invariante arbitraria en $\mathcal{M}_{d}(\mathbb{C})$, para nuestro resultado definimos la distancia $d_{N}(A, B): \mathcal{O}_{B} \rightarrow \mathbb{R}_{>0}$ dada por

$$
d_{N}(A, B)=N\left(\log \left(A^{-1 / 2} B A^{-1 / 2}\right)\right) .
$$

Con esta métrica, el problema de aproximación en $\mathcal{P}(d)$ consiste en calcular

$$
\min _{C \in \mathcal{O}_{B}} F_{N}(C):=\min _{C \in \mathcal{O}_{B}} N\left(\log \left(A^{-1 / 2} C A^{-1 / 2}\right)\right)
$$

y caracterizar sus aproximantes.

Teorema 5.3.2. Sean $A, B \in \mathcal{P}(d)$ y $N(\cdot)$ una $N U I$ estrictamente convexa en $\mathcal{M}_{d}(\mathbb{C})$ consideremos $F_{(N, A, B)}=F_{N}: \mathcal{O}_{B} \rightarrow \mathbb{R}_{>0}$ dado por

$$
F_{N}(C)=N\left(\log \left(A^{-1 / 2} C A^{-1 / 2}\right)\right) .
$$

Entonces, si $C_{0} \in \mathcal{O}_{B}$ es un minimizador local de $F_{N}$, existe $U \in \mathcal{U}(d)$ tal que

$$
U^{*} A U=D_{\lambda(A)} y U^{*} D_{\lambda(B)} U=C_{0} .
$$


En particular, $C_{0}$ resulta ser minimizador global de $F_{N}$ en $\mathcal{O}_{B}$.

Demostración. Supongamos que no existe $U \in \mathcal{U}(d)$ tal que

$$
U^{*} A U=D_{\lambda(A)} \quad \text { y } \quad C_{0}=U^{*} B U=D_{\lambda(B)} .
$$

Del Teorema 5.1.4 tenemos que

$$
\left(\log \left(\lambda\left(C_{0}\right)\right)-\log (\lambda(A))\right)^{\downarrow} \neq \log \left(\lambda\left(C_{0} A^{-1}\right)\right) .
$$

Luego, el Teorema 5.2.7 implica que, para algún $t_{0}>0$ suficientemente pequeño, existe una curva continua

$$
\gamma(t):\left(-t_{0}, t_{0}\right) \longrightarrow \mathcal{U}(d) \text { tal que } \gamma(0)=I
$$

y para $t \neq 0$

$$
\log \lambda\left(A^{-1} C_{0}\right) \neq \log \lambda\left(A^{-1 / 2} \gamma(t) C_{0} \gamma(t)^{*} A^{-1 / 2}\right) \prec \log \lambda\left(A^{-1 / 2} C_{0} A^{-1 / 2}\right) .
$$

Entonces, del Teorema 1.5.7, el hecho que $N(\cdot)$ sea una NUI estrictamente convexa implica que

$$
N\left(\log \left(A^{-1 / 2} \gamma(t) C_{0} \gamma(t)^{*} A^{-1 / 2}\right)\right)<N\left(\log \left(A^{-1 / 2} C_{0} A^{-1 / 2}\right)\right),
$$

lo que contradice que $C_{0}$ sea un minimizador local de $F_{N}$ en $\mathcal{O}_{B}$.

Nuestras conclusiones para extremos locales se pueden aplicar directamente al problema de Procusto usando la métrica de Bures-Wasserstein y la divergencia de Kullback-Leibler ya que esos resultados también dependen de la caracterización de extremos locales/globales para la desigualdad de Gelfand, Naimark y Lidskii. 


\section{Bibliografía}

[1] A. Aldroubi, C. Cabrelli, U. M. Molter, Wavelets on irregular grids with arbitrary dilation matrices and frame atoms for $L^{2}\left(\mathbb{R}^{d}\right)$. Applied and Computational Harmonic Analysis 17 (2004) 119-140.

[2] T. Ando, Majorization, doubly stochastic matrices and comparison of eigenvalues, Linear Algebra and Its Applications, 1 18(1989) 163-248.

[3] J. Antezana, G. Corach, D. Stojanoff and M. Ruiz, Weighted projections and Riesz frames, Lin. Alg. Appl. 402 no. 1-3 (2005)

[4] J. Antezana, G. Corach, M. Ruiz and D. Stojanoff, Nullspaces and frames, J. Math. Anal. Appl. 309 (2005) 709-723

[5] J. Antezana, P. Massey, M. Ruiz and D. Stojanoff, The Schur-Horn theorem for operators and frames with prescribed norms and frame operator, Illinois J. Math., 51 (2007), 537-560.

[6] M.S. Asgari, New characterizations of fusion frames, Proc. Indian Acad. Sci. Math. Sci 119(3), (2009) 369-382.

[7] J. Benedetto, M. Fickus, Finite normalized tight frames, Adv. Comput. Math. 18, No. 2-4 (2003), 357-385 .

[8] R. Bhatia, Matrix analysis, Berlin-Heildelberg-New York , Springer (1997).

[9] R. Bhatia, M. Congedo, Procustes problems in Riemannian manifolds of positive definite matrices. Linear Algebra and its Applications, 563 (2019) 440-445.

[10] R. Bhatia, Linear Algebra to Quantum Cohomology: The Story of Alfred Horn's Inequalities, The American Mathematical Monthly, 108:4 (2001) 289-318.

[11] R. Balan, P. Casazza, D. Edidin, (2005). On signal reconstruction from absolute value of frame coefficients. Proceedings of SPIE - The International Society for Optical Engineering. 5914. 10.1117/12.613965. 
[12] R. Balan, B. Bodmann, P. Casazza, D. Edidin, Painless reconstruction from magnitudes of frame coefficients, J. Fourier Anal. Appl., 15 (2009), 488-501.

[13] A. S. Bandeira, J. Cahill, D. G. Mixon y A. A. Nelson, Saving phase: Injectivity and stability for phase retrieval, Applied and Computational Harmonic Analysis, 37 (1), (2014), 106-125

[14] R. Bates, D. Mnyama, The Status of Practical Fourier Phase Retrieval. Advances in Electronics and Electron Physics, 1-64 (1986).

[15] T. Bemrose, P. Casazza, K. Gröchenig, M. Lammers, R. Lynch, Weaving frames. Oper. Matrices 10 (2016), no. 4, 1093-1116.

[16] J. Benedetto, M. Fickus, Finite normalized tight frames, Adv. Comput. Math. 18, No. 2-4 (2003), 357-385.

[17] J. Benedetto, M. Frazier, Wavelets Mathematics and Applications, CRC Press (1993)

[18] R. Bhatia, Matrix Analysis, Graduate Texts in Mathematics, 169. Springer-Verlag, New York, 1997.

[19] B. Bodmann, P. Casazza, The road to equal-norm Parseval frames, Journal of Functional Analysis 258 (2010) 397-420

[20] B. Bodmann, P. Casazza, V. Paulsen, D. Speegle, Spanning and independence properties of frame partitions, Proc. AMS 1407 (2012), 2193-2207.

[21] J. Cahill, P. Casazza, J. Peterson y L. Woodland, Phase retrieval by projections, preprint.

[22] P. Calderón, N. Rios, M. Ruiz, Local extrema for Procrustes problems in the set of positive definite matrices, Linear Algebra and its Applications 602 (2020) 252-263

[23] P. Calderón, M. Ruiz, A duality result on frames and Riesz sequences. Proceedings of VII MACI 7 (2019) 9-12.

[24] P. Calderón, M. Ruiz, A note on perturbations of fusion frames, J. Math. Anal. Appl. 461 (2018) 169-175.

[25] P. Calderón, M. Ruiz, On perturbations of woven pairs of frames, J. Anal. (2022) https://doi.org/10.1007/s41478-022-00389-y

[26] E. Candès, Y. Eldar, T. Strohmer, V. Voroninski, Phase Retrieval via Matrix Completion, SIAM J. Imaging Sci., 6 (2013), 199-225. 
[27] P.G. Casazza, O. Christensen, S. Li, and A. Linder, Riesz-Fischer Sequences and Lower Frame Bounds, Journal for Analysis and its Applications, 21 No.2 (2002) 305-314.

[28] P. Casazza, D. Freeman, R. Lynch, Weaving Schauder frames, J. Approximation Theory, 211 (2016) 42-60.

[29] P. Casazza, G. Kutyniok, Frames of subspaces. Wavelets, frames and operator theory, 87-113, Contemp. Math., 345, Amer. Math. Soc., Providence, RI, 2004.

[30] P. Casazza, G. Kutyniok, Robustness of Fusion Frames under Erasures of Subspaces and of Local Frame Vectors, preprint.

[31] P. Casazza, G. Kutyniok, S. Li, Fusion frames and distributed processing, Applied and Computational Harmonic Analysis, Volume 25, 2008, 114132.

[32] Casazza, P. G., Lynch, R. G. (2015). Weaving properties of Hilbert space frames. 2015 International Conference on Sampling Theory and Applications (SampTA)

[33] E. Chiumiento, Global Symmetric Approximation of Frames, J. Fourier Anal. Appl. 25 (2019), no. 8, 1395-1423.

[34] O. Christensen, An introduction to frames and Riesz bases. Applied and Numerical Harmonic Analysis. Birkhäuser Boston, 2003. xxii +440 pp.

[35] O. Christensen, Frames and pseudo-inverses. J. Math. Anal. Appl. 195(1995), 401-414

[36] O. Christensen, A Paley-Wiener theorem for frames. Proc. Am. Math. Soc. 123 (1995) 2199-2202

[37] O. Christensen, (1995). Frame Perturbations. Proceedings of the American Mathematical Society, 123(4), 1217. doi:10.2307/2160722

[38] O. Christensen, Operators with Closed Range, Pseudo-Inverses, and Perturbation of Frames for a Subspace, Canad. Math. Bull. Vol. 42 (1), 1999 pp. $37-45$

[39] O. Christensen, C. Heil, Perturbations of Banach frames and atomic decompositions. Math. Nachr. 185 (1997) 33-47

[40] O. Christensen, Y.C. Eldar, Oblique dual frames and shift-invariant spaces, Appl. Comput. Harmon. Anal. 17 (2004)48-68. 
[41] G. Corach, A. Maestripieri and D. Stojanoff, Schur complements and oblique projections. Acta Sci. Math 67 (2001), 439-459.

[42] G. Corach, P. Massey and M. Ruiz, Procrustes problems and Parseval quasi-dual frames, Acta Applicandae Mathematicae 131 (2014), 179-195.

[43] Daubechies, Ingrid and Grossmann, Alexandre and Meyer, Y.J., Painless Nonorthogonal Expansions, J. Math. Physics 27(5) (1986).

[44] I. Daubechies, A. Grossman, Y. Meyer, Painless nonorthogonal expansions, Journ. Math. Phys. 27 (1986), 1271-1283.

[45] Deepshikha, S. Garg, L. Vashisht, G. Verma, On weaving fusion frames for Hilbert spaces. International Conference on Sampling Theory and Applications (SampTA) (2017)

[46] J. Ding and L. J. Huang, Perturbation of generalized inverses of linear operators in Hilbert spaces. J. Math. Anal. Appl. 198(1996), 505-516.

[47] F. Deutsch, The angle between subspaces in Hilbert space, Approximation theory, wavelets and applications (1995) 107-130.

[48] D. Donoho, Unconditional Bases are Optimal Bases for Data Compression and for Statistical Estimation, Applied and Computational Harmonic Analysis 1 (1993), 100-115.

[49] R. G. Douglas; On majorization, factorization and range inclusion of operators in Hilbert spaces. Proc. Am. Math. Soc. 17 (1966) 413-416.

[50] R.J. Duffin, A. C, Schaeffer, A class of nonharmonic Fourier series, Trans. Amer. Math. Soc. 72 (1952) 341-366

[51] Y. Eldar, Sampling with arbitrary sampling and reconstruction spaces and oblique dual frame vectors, J. FourierAnal. Appl. 9 (2003) 77-96.

[52] L. Eldén, H. Park, A Procrustes problem on the Stiefel manifold.Numer. Math. 82, no. 4, 599-619 (1999).

[53] M. Fickus, D. Mixon, A. Nelson y Yang Wang, Phase retrieval from very few measurements, Linear Algebra Appl. 449 (2014), 475-499.

[54] M. Fickus, D.G. Mixon, M.J. Poteet, Frame completions for optimally robust reconstruction, Proc. of SPIE, 8138(1)-SPIE (2011), 1-8.

[55] J. R. Fienup, . Reconstruction of an object from the modulus of its Fourier transform. Optics Letters, 3(1), 27 (1978). doi:10.1364/ol.3.000027

[56] J. R. Fienup, Phase retrieval algorithms: a comparison. Applied Optics, 21(15) (1982), 2758. doi:10.1364/ao.21.002758 
[57] M. Fornasier, Decompositions of Hilbert Spaces: Local Construction of Global Frames, Constructive theory of functions, 2003, pp. 275-281.

[58] M. Fornasier, Quasi-orthogonal decompositions of structured frames. Journal of Mathematical Analysis and Applications 289 (2004) 180-199.

[59] M. Frank, D.R. Larson, Frames in Hilbert $C^{*}$-modules and $C^{*}$-algebras J. Oper. Theory 48 (2) (2002), 273-314.

[60] P. Gavruta, On the duality of fusion frames, J. Math. Anal. Appl. 333 (2007) 871-879.

[61] I. Gel'fand, M. Naimark, The relation between the unitary representations of the complex unimodular group and its unitary subgroup, lzv Akad. Nauk SSSR Ser. Mat. 14 (1950) 239-260.

[62] J. Giribet, A. Maestripieri, F. Martínez Pería, P. Massey, On frames for Krein spaces, J. Math. Anal. Appl. 393 (2012) 122-137

[63] A. R. Gonsalves, Phase retrieval by differential intensity measurements. Journal of the Optical Society of America A, 4(1), 166. doi:10.1364/josaa.4.000166 (1987).

[64] J. C. Gower, G. B. Dijksterhuis, Procrustes problems, Oxford Statistical Science Series, 30. Oxford University Press, Oxford, (2004)

[65] K. Gröchenig, Describing functions: Atomic decompositions versus frames, Monatshefte fur Math. 112 (1991), 1-41.

[66] P. Halmos, A Hilbert Space Problem Book [2nd ed., rev. and enl. ed] (1974)

[67] G.H. Hardy, J.E. Littlewood, G. Polya, Inequalities, Cambridge University Press, (1934).

[68] C. Heil, D. Walnut, Continuous and discrete wavelet transforms. SIAM Review, 31 SIAM Review (1989) 628-666.

[69] N.J. Higham, Matrix nearness problems and applications. Applications of matrix theory (Bradford, 1988), 1-27, Inst. Math. Appl. Conf. Ser. New Ser., 22, Oxford Univ. Press, New York, 1989.

[70] N. J. Higham, The symmetric procrustes problem. BIT 28 (1988) 133-143.

[71] A. Horn, Eigenvalues of sums of Hermitian matrices, Pacific J. Math. 12 (1962), 225-241. 
[72] R.A. Horn, C.R. Johnson, Matrix analysis. Second edition. Cambridge University Press, Cambridge, 2013.

[73] R.A. Horn, C.R. Johnson, Topics in matrix analysis. Corrected reprint of the 1991 original. Cambridge University Press, Cambridge, 1994.

[74] Y. Huang, Y. Yang, K-g-fusion frames in Hilbert spaces, Journal of Inequalities and Applications (2020)

[75] T. Kato, Perturbation theory for nullity, deficiency and other quantities of linear operators. Journal d'Analyse Mathématique, 6(1) (1958) $261-322$.

[76] T. Kato, Perturbation Theory for Linear Operators, Springer-Verlag, Berlin Heidelberg New York 1980.

[77] U. Kintzel, Procrustes problems in finite dimensional indefinite scalar product spaces. Linear Algebra Appl. 402, 1-28 (2005).

[78] A.A. Klyachko, Random walks on symmetric spaces and inequalities for matrix spectra, in: Special Issue: Workshop on Geometric and Combinatorial Methods in the Hermitian Sum Spectral Problem, Coimbra, 1999, Linear Algebra Appl. 319 (1-3) (2000) 37-59.

[79] R.S. Leite, T.R.W. Richa, C. Tomei, Geometric proofs of some theorems of Schur-Horn type, Linear Algebra Appl. 286 (1999) 149-173

[80] M. Levitin, D. Vassiliev, Operator Theory and Its Applications: In Memory of V. B. Lidskii (1924-2008) American Mathematical Society Translations: 231 (2010)

[81] S. Li, H. Ogawa, Pseudoframes for subspaces with applications, J. Fourier Anal. Appl. 10 (2004) 409-431

[82] D. Li, J. Leng, T. Huang, X. Li, On Weaving g-Frames for Hilbert Spaces. Complex Analysis and Operator Theory, 14(2). doi:10.1007/s11785-02000991-7 2020

[83] V.B. Lidskii, On the characteristic numbers of the sum and product of symmetric matrices, (Russian) Doklady Akad. Nauk SSSR (N.S.) 75, (1950). 769-772.

[84] C.K. Li, R. Mathias, The Lidskii-Mirsky-Wielandt theorem-additive and multiplicative versions, Numer. Math. 81 (1999) 377-413.

[85] X.B. Li, L.Z. Yang, Y.C. Zhu, Some results about operator perturbation of fusion frames in Hilbert spaces, J. Math. Anal. Appl. 421 (2015) 1417 $-1427$. 
[86] S. Mallat, A Wavelet Tour of Signal Processing, Wavelet Analysis \& Its Applications, Academic Press (1999) 2ed.

[87] A.W. Marshall and I. Olkin, Inequalities: Theory of Majorization and Its Applications, Academic Press (1979)

[88] P. Massey, M.A. Ruiz, Tight frame completions with prescribed norms. Sampl. Theory Signal Image Process. 7 (2008) 1-13.

[89] P. Massey, M. Ruiz y D. Stojanoff, The structure of minimizers of the frame potential on fusion frame, Journal of Fourier Analysis and Applications, 16(4) (2010), 514-543.

[90] P. Massey, M. Ruiz, D. Stojanoff, Optimal frame completions. Adv Comput Math 40 (2014) 1011-1042.

[91] P. Massey, M.A. Ruiz, D. Stojanoff, Multiplicative Lidskii's inequalities and optimal perturbation of frames, Linear Algebra Appl., 469 (2015) $539-568$.

[92] P. Massey, N. B. Rios, D. Stojanoff, Frame completions with prescribed norms: local minimizers and applications. Adv. Comput. Math. 44 (2018), no. 1, 51-86.

[93] P. Massey, N. B. Rios, D. Stojanoff, Local Lidskii's theorems for unitarily invariant norms. Linear Algebra Appl. 557 (2018), 34-61.

[94] E. Moghaddam, A. Arefijamaal, (2021). On Excesses and Duality in Woven Frames. arXiv:2101.00710

[95] A. Olevskii, A. Ulanovskii, Functions with Disconnected Spectrum. Sampling, Interpolation, Translates, University Lecture Series, American Mathematical Society, 65 (2016).

[96] A. Pietsch, History of Banach Spaces and Linear Operators, Birkhauser (2007).

[97] M. Ruiz, D. Stojanoff, Some properties of frames of subspaces obtained by operator theory methods, J. Math. Anal. Appl. 343 (2008) 366-378.

[98] W. Sun, G-frames and g-Riesz bases, J. Math. Anal. Appl. 322 (1) (2006) $437-452$.

[99] N. Trendafilov, R. Lipper, The multimode Procrustes problem, Linear Algebra and its Applications 349 (2002) 245-264

[100] L. Vashisht, Deepshikha, Weaving properties of generalized continuous frames generated by an iterated function system, J. Geometry and Physics 110 (2016) 282-295 
[101] A. Walther, The question of phase retrieval in optics, Opt. Acta 10 (1963) 4149 .

[102] G. A. Watson, The solution of orthogonal Procrustes problems for a family of orthogonally invariant norms. Adv. Comput. Math. 2 , no. 4, 393-405 (1994).

[103] H. Wielandt, An extremum property of sums of eigenvalues, Proc. Arner. Math. Soc., 6 (1955) No. I, 105-110.

[104] R. Young, An introduction to nonharmonic Fourier series, Academic Press (1980). 